\title{
Systematic Studies of Early Actinide Complexes: Uranium(IV) Fluoroketimides
}

Eric J. Schelter, Ping Yang, Brian L. Scott, J. D. Thompson, Richard L. Martin, P. Jeffrey Hay*, David E. Morris*, and Jaqueline L. Kiplinger*

Los Alamos National Laboratory, Los Alamos, NM, 87545

Fax: +015056679905; Tel: +01505665 9553;

E-mail: kiplinger@lanl.gov

Electronic Supporting Information:

- General Synthetic Procedures/Materials. (S3-S4)

- Synthetic Details and Characterization for 4-11. (S4-S6)

- Crystallographic Experimental Details for 4-11. (S7)

- Table S1. Mulliken Analysis of the Contributions (\%) to the Molecular Orbitals of $\mathbf{6}$ and $\mathbf{1 1}$ from Analysis of the Alpha-Spin MOs from their DFT Calculations. (S8)

- Table S2. Mulliken Analysis of the Contributions (\%) to the Molecular Orbitals of $\mathbf{6}^{-}$and $\mathbf{1 1}^{-}$from Analysis of the Alpha-Spin MOs from their DFT Calculations. (S9)

- Figure S1: Magnetic Susceptibility Data for $\left(\mathrm{C}_{5} \mathrm{Me}_{5}\right)_{2} \mathrm{U}\left[-\mathrm{N}=\mathrm{C}\left(\mathrm{CH}_{3}\right)\left(3-\mathrm{F}_{-} \mathrm{C}_{6} \mathrm{H}_{4}\right)\right]_{2} \quad$ (5) and $\left(\mathrm{C}_{5} \mathrm{Me}_{5}\right)_{2} \mathrm{U}\left[-\mathrm{N}=\mathrm{C}\left(\mathrm{CH}_{3}\right)\left(3,5-\mathrm{F}_{2}-\mathrm{C}_{6} \mathrm{H}_{3}\right)\right]_{2}(8)$ from 350 to $2 \mathrm{~K}$ at $5 \mathrm{~T}$. (S10)

- Figure S2: Thermal Ellipsoid Plot of $\left(\mathrm{C}_{5} \mathrm{Me}_{5}\right)_{2} \mathrm{U}\left[-\mathrm{N}=\mathrm{C}\left(\mathrm{CH}_{3}\right)\left(2-\mathrm{F}_{-} \mathrm{C}_{6} \mathrm{H}_{4}\right)\right]_{2}$ (4) with Ellipsoids Projected at the $50 \%$ Probability Level. (S11)

- Table S3: Crystal Data and Structure Refinement for $\left(\mathrm{C}_{5} \mathrm{Me}_{5}\right)_{2} \mathrm{U}\left[-\mathrm{N}=\mathrm{C}\left(\mathrm{CH}_{3}\right)\left(2-\mathrm{F}-\mathrm{C}_{6} \mathrm{H}_{4}\right)\right]_{2}(4)$. (S12) 
- Table S4: Bond Distances $[\AA]$ and Angles $\left[^{\circ}\right]$ for $\left(\mathrm{C}_{5} \mathrm{Me}_{5}\right)_{2} \mathrm{U}\left[-\mathrm{N}=\mathrm{C}\left(\mathrm{CH}_{3}\right)\left(2-\mathrm{F}-\mathrm{C}_{6} \mathrm{H}_{4}\right)\right]_{2}$ (4). (S13S15)

- Figure S3: Thermal Ellipsoid Plot of One of the Two Disordered Components in the Structure of $\left(\mathrm{C}_{5} \mathrm{Me}_{5}\right)_{2} \mathrm{U}\left[-\mathrm{N}=\mathrm{C}\left(\mathrm{CH}_{3}\right)\left(4-\mathrm{F}-\mathrm{C}_{6} \mathrm{H}_{4}\right)\right]_{2}(6)$ with Ellipsoids Projected at the $50 \%$ Probability Level. The $\mathrm{U}(1)$ Atom Lies on a Crystallographically Imposed Inversion Center. (S16)

- Table S5: Crystal Data and Structure Refinement for $\left(\mathrm{C}_{5} \mathrm{Me}_{5}\right)_{2} \mathrm{U}\left[-\mathrm{N}=\mathrm{C}\left(\mathrm{CH}_{3}\right)\left(4-\mathrm{F}_{-} \mathrm{C}_{6} \mathrm{H}_{4}\right)\right]_{2}(6)$. (S17)

- Table S6: Bond Distances $[\AA]$ and Angles $\left[^{\circ}\right]$ for $\left(\mathrm{C}_{5} \mathrm{Me}_{5}\right)_{2} \mathrm{U}\left[-\mathrm{N}=\mathrm{C}\left(\mathrm{CH}_{3}\right)\left(4-\mathrm{F}-\mathrm{C}_{6} \mathrm{H}_{4}\right)\right]_{2}(6)$. ( $\mathrm{S} 18$ S24)

- Figure S4: Thermal Ellipsoid Plot of One of the Two Symmetry-Independent Molecules in the Asymmetric Unit of $\left(\mathrm{C}_{5} \mathrm{Me}_{5}\right)_{2} \mathrm{U}\left[-\mathrm{N}=\mathrm{C}\left(\mathrm{CH}_{3}\right)\left(2,6-\mathrm{F}_{2}-\mathrm{C}_{6} \mathrm{H}_{3}\right)\right]_{2}(7)$ with Ellipsoids Projected at the $50 \%$ Probability Level. (S25)

- Table S7: Crystal Data and Structure Refinement for $\left(\mathrm{C}_{5} \mathrm{Me}_{5}\right)_{2} \mathrm{U}\left[-\mathrm{N}=\mathrm{C}\left(\mathrm{CH}_{3}\right)\left(2,6-\mathrm{F}_{2}-\mathrm{C}_{6} \mathrm{H}_{4}\right)\right]_{2}$ (7). (S26)

- Table S8: Bond Distances $[\AA]$ and Angles $\left[^{\circ}\right]$ for $\left(\mathrm{C}_{5} \mathrm{Me}_{5}\right)_{2} \mathrm{U}\left[-\mathrm{N}=\mathrm{C}\left(\mathrm{CH}_{3}\right)\left(2,6-\mathrm{F}_{2}-\mathrm{C}_{6} \mathrm{H}_{4}\right)\right]_{2}$ (7). (S27S33)

- Table S9: Crystal Data and Structure Refinement for $\left(\mathrm{C}_{5} \mathrm{Me}_{5}\right)_{2} \mathrm{U}\left[-\mathrm{N}=\mathrm{C}\left(\mathrm{CH}_{3}\right)\left(2,4,6-\mathrm{F}_{3}-\mathrm{C}_{6} \mathrm{H}_{3}\right)\right]_{2}$ (9). (S34)

- Table S10: Bond Distances $[\AA]$ and Angles $\left[^{\circ}\right]$ for $\left(\mathrm{C}_{5} \mathrm{Me}_{5}\right)_{2} \mathrm{U}\left[-\mathrm{N}=\mathrm{C}\left(\mathrm{CH}_{3}\right)\left(2,4,6-\mathrm{F}_{3}-\mathrm{C}_{6} \mathrm{H}_{3}\right)\right]_{2}$ (9). (S35-S37)

- Table S11: Crystal Data and Structure Refinement for $\left(\mathrm{C}_{5} \mathrm{Me}_{5}\right)_{2} \mathrm{U}\left[-\mathrm{N}=\mathrm{C}\left(\mathrm{CH}_{3}\right)\left(3,4,5-\mathrm{F}_{3}-\mathrm{C}_{6} \mathrm{H}_{3}\right)\right]_{2}$ (10). (S38)

- Table S12: Bond Distances $[\AA]$ and Angles $\left[^{\circ}\right]$ for $\left(\mathrm{C}_{5} \mathrm{Me}_{5}\right)_{2} \mathrm{U}\left[-\mathrm{N}=\mathrm{C}\left(\mathrm{CH}_{3}\right)\left(3,4,5-\mathrm{F}_{3}-\mathrm{C}_{6} \mathrm{H}_{3}\right)\right]_{2}(\mathbf{1 0})$. (S39-S41)

- Figure S5: Thermal Ellipsoid Plot of $\left(\mathrm{C}_{5} \mathrm{Me}_{5}\right)_{2} \mathrm{U}\left[-\mathrm{N}=\mathrm{C}\left(\mathrm{CH}_{3}\right)\left(\mathrm{C}_{6} \mathrm{~F}_{5}\right)\right]_{2}$ (11) with Ellipsoids Projected at the $50 \%$ Probability Level. (S42)

- Table S13: Crystal Data and Structure Refinement for $\left(\mathrm{C}_{5} \mathrm{Me}_{5}\right)_{2} \mathrm{U}\left[-\mathrm{N}=\mathrm{C}\left(\mathrm{CH}_{3}\right)\left(\mathrm{C}_{6} \mathrm{~F}_{5}\right)\right]_{2}(\mathbf{1 1})$. (S43)

- Table S14: Bond Distances $[\AA]$ and Angles [ $\left.{ }^{\circ}\right]$ for $\left(\mathrm{C}_{5} \mathrm{Me}_{5}\right)_{2} \mathrm{U}\left[-\mathrm{N}=\mathrm{C}\left(\mathrm{CH}_{3}\right)\left(\mathrm{C}_{6} \mathrm{~F}_{5}\right)\right]_{2}$ (11). (S44-S48) 


\section{Supporting Information: Experimental}

General Synthetic Procedures. Unless otherwise noted, reactions and manipulations were performed at $20{ }^{\circ} \mathrm{C}$ in a recirculating Vacuum Atmospheres Model HE-553-2 inert atmosphere $\left(\mathrm{N}_{2}\right.$ or $\left.\mathrm{He}\right)$ drybox with a MO-40-2 Dri-Train, or using standard Schlenk and high vacuum line techniques. Glassware was dried overnight at $150{ }^{\circ} \mathrm{C}$ before use. All NMR spectra were obtained in either toluene- $d_{8}$ or benzene- $d_{6}$ using a Bruker Avance $300 \mathrm{MHz}$ spectrometer. Chemical shifts for ${ }^{1} \mathrm{H}$ and ${ }^{13} \mathrm{C}\left\{{ }^{1} \mathrm{H}\right\}$ NMR spectra were referenced to solvent impurities and ${ }^{19} \mathrm{~F}$ NMR resonances were referenced to $\mathrm{CFCl}_{3}$ at $\delta 0.00 \mathrm{ppm}$. Mass spectrometric (MS) analyses were obtained at the University of California, Berkeley Mass Spectrometry Facility, using either VG ProSpec (EI) or VG70-SE (FAB) mass spectrometers. Elemental analyses were performed at the University of California, Berkeley Microanalytical Facility, on a Perkin-Elmer Series II 2400 CHNS analyzer.

Materials. Unless otherwise noted, reagents were purchased from commercial suppliers and used without further purification. Celite (Aldrich) and alumina (Brockman I, Aldrich) were dried under dynamic vacuum at $250{ }^{\circ} \mathrm{C}$ for $48 \mathrm{~h}$ prior to use. Anhydrous toluene (Aldrich), pentane (Aldrich), diethyl ether (Aldrich), and tetrahydrofuran (Aldrich) were passed through a column of activated alumina (A2, $12 \times 32$, Purifry) under nitrogen and stored over activated $4 \AA$ molecular sieves prior to use. Benzene- $d_{6}$ (Aldrich), toluene- $d_{8}$ (Aldrich), 2-fluorobenzonitrile (Aldrich), 3-fluorobenzonitrile (Aldrich), 2,6-difluorobenzonitrile (Aldrich), and pentafluorobenzonitrile (Aldrich) were purified by storage over activated $4 \AA$ molecular sieves under $\mathrm{N}_{2}$ prior to use. $\left(\mathrm{C}_{5} \mathrm{Me}_{5}\right)_{2} \mathrm{U}\left(\mathrm{CH}_{3}\right)_{2}$ was prepared according to literature procedures. ${ }^{1}$ Caution: Depleted uranium (primarily isotope ${ }^{238} \mathrm{U}$ ) is a weak $\alpha$-emitter with a half-life $4.47 \mathrm{x}$ $10^{9}$ years; manipulations and reactions should be carried out in monitored fume hoods or in an 
inert atmosphere drybox in a radiation laboratory equipped with $\alpha$ - and $\beta$-counting equipment.

(1) Fagan, P. J.; Manriquez, J. M.; Maatta, E. A.; Seyam, A. M.; Marks, T. J. J. Am. Chem. Soc. 1981, 103, 6650-6667.

Synthesis of $\left(\mathrm{C}_{5} \mathrm{Me}_{5}\right)_{2} \mathrm{U}\left[-\mathrm{N}=\mathrm{C}\left(\mathrm{CH}_{3}\right)\left(2-\mathrm{F}-\mathrm{C}_{6} \mathrm{H}_{4}\right)\right]_{2}$ (4). Complex 4 was prepared from $\left(\mathrm{C}_{5} \mathrm{Me}_{5}\right)_{2} \mathrm{U}\left(\mathrm{CH}_{3}\right)_{2}(0.328 \mathrm{~g}, 0.609 \mathrm{mmol})$ and 2-fluorobenzonitrile $(0.180 \mathrm{~g}, 1.49 \mathrm{mmol}, 2.45$ equiv). Compound 4 was recrystallized from a cold solution of $25 \mathrm{~mL}$ pentane and $1 \mathrm{~mL}$ toluene. Yield: $0.293 \mathrm{~g}, 0.375 \mathrm{mmol}, 62 \%$. ${ }^{1} \mathrm{H}$ NMR (toluene- $d_{8}, 363 \mathrm{~K}$ ): $\delta 10.25$ (br s, $2 \mathrm{H}$, $\left.\mathrm{C}_{6} \mathrm{H}_{4} \mathrm{~F}\right), 7.51$ (br s, $\left.2 \mathrm{H}, \mathrm{C}_{6} \mathrm{H}_{4} \mathrm{~F}\right), 6.96\left(\mathrm{~m}, 2 \mathrm{H}, \mathrm{C}_{6} \mathrm{H}_{4} \mathrm{~F}\right), 3.55$ (br s, $\left.2 \mathrm{H}, \mathrm{C}_{6} \mathrm{H}_{4} \mathrm{~F}\right),-1.52(\mathrm{~s}, 30 \mathrm{H}$, $\left.\mathrm{C}_{5}\left(\mathrm{CH}_{3}\right)_{5}\right),-4.04\left(\mathrm{~s}, 6 \mathrm{H}, \mathrm{CH}_{3}\right) .{ }^{19} \mathrm{~F}$ NMR (benzene- $\left.d_{6}, 298 \mathrm{~K}\right): \delta-42.00\left(\mathrm{~s}, 2 \mathrm{~F}, o-\mathrm{C}_{6} \mathrm{H}_{4} F\right) . \quad \mathrm{MS}$ (EI, $70 \mathrm{eV}): \mathrm{m} / \mathrm{z} 779.0\left(\mathrm{M}^{+}\right)$. Anal. Calcd. for $\mathrm{C}_{36} \mathrm{H}_{43} \mathrm{~N}_{2} \mathrm{~F}_{2} \mathrm{U}$ (779.78 g/mol): C, 55.45; H, 5.56; N, 3.59. Found: C, 55.38; H, 5.80; N, 3.52.

Synthesis of $\left(\mathrm{C}_{5} \mathrm{Me}_{5}\right)_{2} \mathrm{U}\left[-\mathrm{N}=\mathrm{C}\left(\mathrm{CH}_{3}\right)\left(3-\mathrm{F}-\mathrm{C}_{6} \mathrm{H}_{4}\right)\right]_{2}$ (5). Complex 5 was prepared from $\left(\mathrm{C}_{5} \mathrm{Me}_{5}\right)_{2} \mathrm{U}\left(\mathrm{CH}_{3}\right)_{2}(0.420 \mathrm{~g}, 0.780 \mathrm{mmol})$ and 3-fluorobenzonitrile $(0.201 \mathrm{~g}, 1.66 \mathrm{mmol}, 2.13$ equiv). After removal of the solvent, the tacky solid was dried by exposure to an $\mathrm{N}_{2}$ dry box atmosphere for $16 \mathrm{~h}$. Yield: $0.374 \mathrm{~g}, 0.479 \mathrm{mmol}, 61 \%$. ${ }^{1} \mathrm{H}$ NMR (toluene- $\mathrm{d}_{8}, 363 \mathrm{~K}$ ): $\delta 21.45$ (br s, $2 \mathrm{H}, \mathrm{C}_{6} \mathrm{H}_{4} \mathrm{~F}$ ), 17.53 (br s, 2H, $\mathrm{C}_{6} \mathrm{H}_{4} \mathrm{~F}$ ), 12.60 (br s, 2H, $\mathrm{C}_{6} \mathrm{H}_{4} \mathrm{~F}$ ), 6.69 (br s, 2H, $\mathrm{C}_{6} \mathrm{H}_{4} \mathrm{~F}$ ), -1.79 $\left(\mathrm{s}, 30 \mathrm{H}, \mathrm{C}_{5}\left(\mathrm{CH}_{3}\right)_{5}\right),-7.01$ (s, 6H, $\left.\mathrm{CH}_{3}\right) .{ }^{19} \mathrm{~F}$ NMR (toluene- $d_{8}, 298 \mathrm{~K}$ ): $\delta-44.89$ (br s, $2 \mathrm{~F}, m-$ $\left.\mathrm{C}_{6} \mathrm{H}_{4} F\right)$. MS (EI, $\left.70 \mathrm{eV}\right): \mathrm{m} / \mathrm{z} 780.4\left(\mathrm{M}^{+}\right)$. Multiple elemental analyses for 5 were consistently low in carbon, though the NMR data, and sum of data for 4-11 support the assigned formula.

Synthesis of $\left(\mathrm{C}_{5} \mathrm{Me}_{5}\right)_{2} \mathrm{U}\left[-\mathrm{N}=\mathrm{C}\left(\mathrm{CH}_{3}\right)\left(4-\mathrm{F}-\mathrm{C}_{6} \mathrm{H}_{4}\right)\right]_{2}$ (6). Complex 6 was prepared from $\left(\mathrm{C}_{5} \mathrm{Me}_{5}\right)_{2} \mathrm{U}\left(\mathrm{CH}_{3}\right)_{2}(1.00 \mathrm{~g}, 1.86 \mathrm{mmol})$ and 4-fluorobenzonitrile $(0.47 \mathrm{~g}, 3.88 \mathrm{mmol}, 2.09$ equiv). After removal of solvent, a saturated mixture of the dark brown solid was prepared using $40 \mathrm{~mL}$ hot pentane. This mixture was filtered through a medium porosity frit and the brown solid 
collected and dried under reduced pressure to give the first crop of product $(0.388 \mathrm{~g})$. A second crop of crystals were obtained by cooling the hot pentane solution of 6 to room temperature, followed by slow evaporation of the solution. The crystals were then collected $(0.186 \mathrm{~g})$. Combined yield: $0.574 \mathrm{~g}, 0.736 \mathrm{mmol}, 40 \% .{ }^{1} \mathrm{H}$ NMR (toluene- $d_{8}, 388 \mathrm{~K}$ ): $\delta 17.37$ (br s, $4 \mathrm{H}, p$ $\left.\mathrm{C}_{6} \mathrm{H}_{4} \mathrm{~F}\right), 6.05$ (br s, $\left.4 \mathrm{H}, o_{-} \mathrm{C}_{6} \mathrm{H}_{4} \mathrm{~F}\right),-1.97\left(\mathrm{~s}, 30 \mathrm{H}, \mathrm{C}_{5}\left(\mathrm{CH}_{3}\right)_{5}\right),-3.45\left(\mathrm{~s}, 6 \mathrm{H}, \mathrm{CH}_{3}\right) .{ }^{19} \mathrm{~F}$ NMR (benzene- $\left.d_{6}, 298 \mathrm{~K}\right): \delta-55.61\left(\mathrm{~s}, 2 \mathrm{~F}, p-\mathrm{C}_{6} \mathrm{H}_{4} F\right)$. MS (EI, $\left.70 \mathrm{eV}\right): \mathrm{m} / \mathrm{z} 780\left(\mathrm{M}^{+}\right)$. Anal. Calcd. for $\mathrm{C}_{36} \mathrm{H}_{44} \mathrm{~N}_{2} \mathrm{~F}_{2} \mathrm{U}(780.78 \mathrm{~g} / \mathrm{mol}): \mathrm{C}, 55.38 ; \mathrm{H}, 5.68 ; \mathrm{N}, 3.59$. Found: $\mathrm{C}, 55.42 ; \mathrm{H}, 5.59 ; \mathrm{N}, 3.81$.

Synthesis of $\left(\mathrm{C}_{5} \mathrm{Me}_{5}\right)_{2} \mathrm{U}\left[-\mathrm{N}=\mathrm{C}\left(\mathrm{CH}_{3}\right)\left(2,6-\mathrm{F}_{2}-\mathrm{C}_{6} \mathrm{H}_{3}\right)\right]_{2}$ (7). Complex 7 was prepared from reaction of $\left(\mathrm{C}_{5} \mathrm{Me}_{5}\right)_{2} \mathrm{U}\left(\mathrm{CH}_{3}\right)_{2}(0.427 \mathrm{~g}, 0.793 \mathrm{mmol})$ and 2,6-difluorobenzonitrile $(0.251 \mathrm{~g}, 1.80$ mmol, 2.27 equiv). Red-brown 7 was obtained by pentane trituration $(3 \times 15 \mathrm{~mL})$ of the residue left upon reduction of the pentane filtrate. Yield: $0.203 \mathrm{~g}, 0.249 \mathrm{mmol}, 31 \% .{ }^{1} \mathrm{H}$ NMR (benzene- $\left.d_{6}, 298 \mathrm{~K}\right): \delta 7.44\left(7, J_{1}=7.4 \mathrm{~Hz}, J_{2}=1.8 \mathrm{~Hz}, 2 \mathrm{H}, p-\mathrm{C}_{6} H_{3} \mathrm{~F}_{2}\right), 3.67(\mathrm{t}, J=7.7 \mathrm{~Hz}, 4 \mathrm{H}$, $\left.m-\mathrm{C}_{6} \mathrm{H}_{4} \mathrm{~F}\right),-1.64\left(\mathrm{~s}, 30 \mathrm{H}, \mathrm{C}_{5}\left(\mathrm{CH}_{3}\right)_{5}\right),-7.91\left(\mathrm{~s}, 6 \mathrm{H}, \mathrm{CH}_{3}\right) .{ }^{19} \mathrm{~F}$ NMR (benzene- $\left.d_{6}, 298 \mathrm{~K}\right): \delta-57.84$ (s, $\left.4 \mathrm{~F}, o-\mathrm{C}_{6} \mathrm{H}_{3} F_{2}\right)$. MS (EI, $\left.70 \mathrm{eV}\right): \mathrm{m} / \mathrm{z} 816\left(\mathrm{M}^{+}\right)$. Anal. Calcd. for $\mathrm{C}_{36} \mathrm{H}_{42} \mathrm{~N}_{2} \mathrm{~F}_{4} \mathrm{U}(816.77 \mathrm{~g} / \mathrm{mol})$ : C, 52.94; H, 5.18; N, 3.43. Found: C, 52.88; H, 5.04; N, 3.35.

Synthesis of $\left(\mathrm{C}_{5} \mathrm{Me}_{5}\right)_{2} \mathrm{U}\left[-\mathrm{N}=\mathrm{C}\left(\mathrm{CH}_{3}\right)\left(3,5-\mathrm{F}_{2}-\mathrm{C}_{6} \mathrm{H}_{3}\right)\right]_{2}$ (8). Complex 8 was prepared from reaction of $\left(\mathrm{C}_{5} \mathrm{Me}_{5}\right)_{2} \mathrm{U}\left(\mathrm{CH}_{3}\right)_{2}(0.960 \mathrm{~g}, 1.78 \mathrm{mmol})$ and 3,5-difluorobenzonitrile $(0.509 \mathrm{~g}, 3.66$ mmol, 2.06 equiv). The product is soluble in pentanes. Analytically pure material was obtained by removing the volatiles obtained following filtration, and collecting the black solid. Yield: $0.930 \mathrm{~g}, 1.14 \mathrm{mmol}, 64 \%$. ${ }^{1} \mathrm{H}-\mathrm{NMR}$ (toluene- $\mathrm{d}_{8}, 388 \mathrm{~K}$ ): $\delta 20.15$ (br s, $4 \mathrm{H}, o-\mathrm{C}_{6} \mathrm{H}_{3} \mathrm{~F}_{2}$ ), 12.40 (br s, $\left.2 \mathrm{H}, p-\mathrm{C}_{6} \mathrm{H}_{3} \mathrm{~F}_{2}\right),-1.50\left(\mathrm{~s}, 30 \mathrm{H}, \mathrm{C}_{5}\left(\mathrm{CH}_{3}\right)_{5}\right),-10.45\left(\mathrm{~s}, 6 \mathrm{H}, \mathrm{CH}_{3}\right) .{ }^{19} \mathrm{~F}-\mathrm{NMR}$ (toluene- $\left.d_{8}, 388 \mathrm{~K}\right): \delta$ $-42.10\left(\mathrm{~s}, 4 \mathrm{~F}, m-\mathrm{C}_{6} \mathrm{H}_{3} \mathrm{~F}_{2}\right)$. MS (EI, $\left.70 \mathrm{eV}\right): \mathrm{m} / \mathrm{z} 816\left(\mathrm{M}^{+}\right)$. Anal. Calcd. for $\mathrm{C}_{36} \mathrm{H}_{42} \mathrm{~N}_{2} \mathrm{~F}_{4} \mathrm{U}(816.77$ g/mol): C, 52.94; H, 5.18; N, 3.43. Found: C, 52.64; H, 5.20; N, 3.58 . 
Synthesis of $\left(\mathrm{C}_{5} \mathrm{Me}_{5}\right)_{2} \mathrm{U}\left[-\mathrm{N}=\mathrm{C}\left(\mathrm{CH}_{3}\right)\left(2,4,6-\mathrm{F}_{3}-\mathrm{C}_{6} \mathrm{H}_{2}\right)\right]_{2}$ (9). Compound 9 was prepared from $\left(\mathrm{C}_{5} \mathrm{Me}_{5}\right)_{2} \mathrm{U}\left(\mathrm{CH}_{3}\right)_{2}(0.471 \mathrm{~g}, 0.875 \mathrm{mmol})$ and 2,4,6-trifluorobenzonitrile $(0.313 \mathrm{~g}(1.99 \mathrm{mmol}$, 2.28 equiv) and recrystallized from pentane at $-30{ }^{\circ} \mathrm{C}$. Yield: $0.441 \mathrm{~g}, 0.517 \mathrm{mmol}, 59 \% .{ }^{1} \mathrm{H}$ NMR (benzene- $\left.d_{6}, 298 \mathrm{~K}\right): \delta 2.47\left(\mathrm{t}, J_{\mathrm{HF}}=8.3 \mathrm{~Hz}, 4 \mathrm{H}, m-\mathrm{C}_{6} \mathrm{H}_{2} \mathrm{~F}_{3}\right),-1.73\left(\mathrm{~s}, 30 \mathrm{H}, \mathrm{C}_{5}\left(\mathrm{CH}_{3}\right)_{5}\right),-$ $4.21\left(\mathrm{~s}, 6 \mathrm{H}, \mathrm{CH}_{3}\right) .{ }^{19} \mathrm{~F}$ NMR (benzene- $\left.d_{6}, 298 \mathrm{~K}\right): \delta-53.88\left(\mathrm{~m}, 2 \mathrm{~F}, p-\mathrm{C}_{6} \mathrm{H}_{2} \mathrm{~F}_{3}\right),-56.49$ (s, 4F, o$\left.\mathrm{C}_{6} \mathrm{H}_{2} F_{3}\right)$. MS (EI, $\left.70 \mathrm{eV}\right): \mathrm{m} / \mathrm{z} 852\left(\mathrm{M}^{+}\right)$. Anal. Calcd. for $\mathrm{C}_{36} \mathrm{H}_{40} \mathrm{~N}_{2} \mathrm{~F}_{6} \mathrm{U}(852.75 \mathrm{~g} / \mathrm{mol}): \mathrm{C}$, 50.71; H, 4.73; N, 3.29. Found: C, 50.78; H, 4.66; N, 3.20 .

Synthesis of $\left(\mathrm{C}_{5} \mathrm{Me}_{5}\right)_{2} \mathrm{U}\left[-\mathrm{N}=\mathrm{C}\left(\mathrm{CH}_{3}\right)\left(3,4,5-\mathrm{F}_{3}-\mathrm{C}_{6} \mathrm{H}_{2}\right)\right]_{2}$ (10). Dark red-brown 10 was prepared from $\left(\mathrm{C}_{5} \mathrm{Me}_{5}\right)_{2} \mathrm{U}\left(\mathrm{CH}_{3}\right)_{2}(0.301 \mathrm{~g}, 0.559 \mathrm{mmol})$ and 3,4,5-trifluorobenzonitrile $(0.193 \mathrm{~g}, 1.230$ mmol, 2.2 equiv). Analytically pure material was obtained by crystallization from saturated pentane solutions of 10 at $-30{ }^{\circ} \mathrm{C}$. Yield: $0.310 \mathrm{~g}, 0.364 \mathrm{mmol}, 65 \%$. ${ }^{1} \mathrm{H}-\mathrm{NMR}$ (toluene- $\mathrm{d}_{8}, 388$ $\mathrm{K}): \delta 19.29\left(\mathrm{br} \mathrm{s}, 4 \mathrm{H}, o-\mathrm{C}_{6} \mathrm{H}_{2} \mathrm{~F}_{3}\right),-1.47\left(\mathrm{~s}, 30 \mathrm{H}, \mathrm{C}_{5}\left(\mathrm{CH}_{3}\right)_{5}\right),-9.55\left(\mathrm{br} \mathrm{s}, 6 \mathrm{H}, \mathrm{CH}_{3}\right) .{ }^{19} \mathrm{~F}-\mathrm{NMR}$ (benzene- $d_{6}, 298 \mathrm{~K}$ ): $\delta-65.18$ (br s, 4F, $p-\mathrm{C}_{6} \mathrm{H}_{2} F_{3}$ ), $-129.16\left(\mathrm{~s}, 2 \mathrm{~F}, o-\mathrm{C}_{6} \mathrm{H}_{2} F_{3}\right) . \quad$ MS (EI, $70 \mathrm{eV}$ ): m/z 852.4 $\left(\mathrm{M}^{+}\right)$. Anal. Calcd. for $\mathrm{C}_{36} \mathrm{H}_{40} \mathrm{~N}_{2} \mathrm{~F}_{6} \mathrm{U}(852.75 \mathrm{~g} / \mathrm{mol})$ : C, 50.71; H, 4.73; N, 3.29. Found: C, 50.40; H, 4.69; N, 3.16 .

Synthesis of $\left(\mathrm{C}_{5} \mathrm{Me}_{5}\right)_{2} \mathrm{U}\left[-\mathrm{N}=\mathrm{C}\left(\mathrm{CH}_{3}\right)\left(\mathrm{C}_{6} \mathrm{~F}_{5}\right)\right]_{2}$ (11). Complex 11 was prepared from reaction of $\left(\mathrm{C}_{5} \mathrm{Me}_{5}\right)_{2} \mathrm{U}\left(\mathrm{CH}_{3}\right)_{2}(0.542 \mathrm{~g}, 0.93 \mathrm{mmol})$ and pentafluorobenzonitrile $(0.423 \mathrm{~mL}, 2.19 \mathrm{mmol}, 2.35$ equiv). Analytically pure material was obtained by crystallization from saturated pentane solutions of 11 at $-30{ }^{\circ} \mathrm{C}$. Yield: $0.682 \mathrm{~g}, 0.74 \mathrm{mmol}, 79 \%$. ${ }^{1} \mathrm{H}$ NMR (benzene- $d_{6}, 298 \mathrm{~K}$ ): $\delta-$ $1.19\left(\mathrm{~s}, 6 \mathrm{H}, \mathrm{CH}_{3}\right),-1.44\left(\mathrm{~s}, 30 \mathrm{H}, \mathrm{C}_{5}\left(\mathrm{CH}_{3}\right)_{5}\right) .{ }^{19} \mathrm{~F}$ NMR (benzene- $\left.d_{6}, 297 \mathrm{~K}\right): \delta-91.68$ (dd, 4F, o$\left.\mathrm{C}_{6} \mathrm{~F}_{5}\right),-102.81\left(\mathrm{~m}, 6 \mathrm{~F}, \mathrm{~m}, p-\mathrm{C}_{6} \mathrm{~F}_{5}\right)$. MS (EI, $\left.70 \mathrm{eV}\right): \mathrm{m} / \mathrm{z} 924\left(\mathrm{M}^{+}\right)$. Anal. Calcd. for $\mathrm{C}_{36} \mathrm{H}_{36} \mathrm{~N}_{2} \mathrm{~F}_{10} \mathrm{U} \cdot 1 / 2 \mathrm{C}_{5} \mathrm{H}_{12}(960.78 \mathrm{~g} / \mathrm{mol}): \mathrm{C}, 48.13 ; \mathrm{H}, 4.41 ; \mathrm{N}, 2.92$. Found: $\mathrm{C}, 48.08 ; \mathrm{H}, 4.40$; N, 2.97 . 
Experimental Crystallographic Details: The crystal structures of compounds 4, 6, 7, 9, 10, and 11 were determined as follows, with exceptions noted in subsequent paragraphs: The crystal was mounted in a nylon cryoloop from Paratone-N oil under argon gas flow. The data were collected on a Bruker SMART APEX II charge-coupled-device (CCD) diffractometer, with KRYO-FLEX liquid nitrogen vapor cooling device. The instrument was equipped with graphite monochromatized MoK $\alpha$ X-ray source $(\lambda=0.71073 \AA)$, with MonoCap X-ray source optics. A hemisphere of data was collected using $\omega$ scans, with 5 -second frame exposures and $0.3^{\circ}$ frame widths. Data collection and initial indexing and cell refinement were handled using APEX II software. ${ }^{1}$ Frame integration, including Lorentz-polarization corrections, and final cell parameter calculations were carried out using SAINT + software. $^{2}$ The data were corrected for absorption using the SADABS program. ${ }^{3}$ Decay of reflection intensity was monitored by analysis of redundant frames. The structure was solved using Direct methods and difference Fourier techniques. All hydrogen atom positions were idealized contributions. The final refinement included anisotropic temperature factors on all non-hydrogen atoms. Structure solution, refinement, graphics, and creation of publication materials were performed using SHELXTL. ${ }^{4}$

Compound 11: Two independent molecules per unit cell were refined. One of the $\mathrm{C}_{5} \mathrm{Me}_{5}$ ligands on the first molecule (U1 metal center) was disordered, and refined as two one-half occupancy $\mathrm{C}_{5} \mathrm{Me}_{5}$ ligands. Each $\mathrm{C}_{5} \mathrm{Me}_{5}$ ligand was refined as a rigid body. The atomic displacement parameters on corresponding pairs of disordered atomic positions were restrained to be equivalent.

\section{$\underline{\text { References }}$}

1. APEX II 1.08, 2004, Bruker AXS, Inc., Madison, Wisconsin 53719.

2. SAINT+ 7.06, 2003, Bruker AXS, Inc., Madison, Wisconsin 53719.

3. SADABS 2.03, 2001, George Sheldrick, University of Göttingen, Germany.

4. SHELXTL 5.10, 1997, Bruker AXS, Inc., Madison, Wisconsin 53719. 
Table S1: Mulliken Analysis of the Contributions (\%) to the Molecular Orbitals of $\mathbf{6}$ and $\mathbf{1 1}$ from Analysis of the Alpha-Spin MOs from their DFT Calculations.

\begin{tabular}{|c|c|c|c|c|c|c|c|c|}
\hline \multirow{2}{*}{ Orbital } & \multirow{2}{*}{$E(e V)$} & \multicolumn{3}{|c|}{$\mathrm{U}$} & \multicolumn{2}{|c|}{$\mathrm{C}=\mathrm{N}$} & \multirow{2}{*}{$\operatorname{Ar}_{\mathrm{F}}$} & \multirow{2}{*}{$\mathrm{C}_{5} \mathrm{Me}_{5}$} \\
\hline & & $\mathrm{sp}$ & d & $\mathrm{f}$ & $\mathrm{N}$ & $\mathrm{C}$ & & \\
\hline \multicolumn{9}{|l|}{6} \\
\hline LUMO+8 & -0.20 & 0.0 & 0.2 & 0.3 & 0.4 & 0.0 & 97.9 & 0.8 \\
\hline LUMO+7 & -0.35 & 0.0 & 0.3 & 0.3 & 0.2 & 0.0 & 98.1 & 0.7 \\
\hline LUMO+6 & -0.63 & 0.7 & 4.1 & 47.0 & 9.2 & 4.5 & 25.1 & 8.4 \\
\hline LUMO+5 & -0.75 & 3.4 & 2.9 & 39.5 & 10.6 & 5.3 & 32.0 & 5.7 \\
\hline LUMO+4 & -1.20 & 0.3 & 4.8 & 53.9 & 11.5 & 7.3 & 15.1 & 5.3 \\
\hline $\mathrm{LUMO}+3$ & -1.31 & 1.4 & 6.8 & 67.7 & 7.1 & 4.4 & 7.6 & 3.9 \\
\hline LUMO+2 & -1.37 & 5.3 & 5.4 & 75.7 & 3.5 & 2.1 & 3.8 & 3.4 \\
\hline LUMO+1 & -1.53 & 0.8 & 5.6 & 67.6 & 4.9 & 5.9 & 8.7 & 5.4 \\
\hline LUMO & -1.59 & 0.7 & 3.1 & 74.1 & 3.5 & 5.5 & 7.5 & 4.0 \\
\hline HOMO & -4.59 & 0.3 & 5.6 & 57.3 & 4.7 & 5.3 & 4.6 & 21.1 \\
\hline HOMO-1 & -4.68 & 0.6 & 3.3 & 60.0 & 2.7 & 6.9 & 5.4 & 19.5 \\
\hline HOMO-2 & -5.18 & 1.7 & 1.6 & 8.7 & 1.4 & 1.1 & 1.1 & 84.1 \\
\hline HOMO-3 & -5.31 & 2.4 & 5.2 & 4.2 & 7.8 & 1.8 & 3.0 & 74.1 \\
\hline HOMO-4 & -5.42 & 0.2 & 1.3 & 19.3 & 45.9 & 2.1 & 5.4 & 19.9 \\
\hline HOMO-5 & -5.70 & 0.3 & 7.9 & 26.5 & 5.0 & 0.6 & 1.4 & 57.5 \\
\hline HOMO-6 & -5.82 & 0.3 & 5.8 & 32.8 & 11.8 & 0.6 & 2.0 & 45.4 \\
\hline \multicolumn{9}{|l|}{11} \\
\hline $\mathrm{LUMO}+8$ & -0.14 & 0.1 & 1.5 & 1.0 & 1.9 & 3.4 & 90.4 & 0.8 \\
\hline LUMO+7 & -0.31 & 0.3 & 0.4 & 0.0 & 0.0 & 0.2 & 98.3 & 0.4 \\
\hline LUMO+6 & -0.67 & 0.1 & 2.5 & 6.7 & 8.6 & 8.2 & 69.7 & 3.2 \\
\hline LUMO+5 & -0.74 & 0.1 & 1.4 & 7.6 & 4.8 & 4.4 & 78.6 & 1.4 \\
\hline LUMO+4 & -0.95 & 0.2 & 4.8 & 77.7 & 4.6 & 2.6 & 2.6 & 5.8 \\
\hline $\mathrm{LUMO}+3$ & -1.31 & 0.1 & 7.0 & 84.6 & 1.0 & 0.2 & 1.2 & 5.8 \\
\hline LUMO+2 & -1.44 & 10.0 & 5.4 & 73.1 & 2.4 & 4.6 & 2.4 & 1.2 \\
\hline LUMO+1 & -1.89 & 0.3 & 2.9 & 90.7 & 0.4 & 1.0 & 0.0 & 4.0 \\
\hline LUMO & -1.90 & 0.0 & 5.4 & 77.1 & 4.0 & 4.5 & 2.4 & 5.4 \\
\hline HOMO & -4.90 & 0.3 & 5.9 & 65.0 & 4.2 & 6.6 & 1.6 & 14.8 \\
\hline HOMO-1 & -4.95 & 0.2 & 2.7 & 73.9 & 6.4 & 4.4 & 1.0 & 9.9 \\
\hline HOMO-2 & -5.25 & 1.9 & 1.1 & 7.4 & 1.4 & 0.4 & 0.0 & 87.8 \\
\hline HOMO-3 & -5.33 & 2.6 & 4.6 & 6.3 & 6.8 & 1.4 & 0.6 & 76.8 \\
\hline HOMO-4 & -5.66 & 0.2 & 2.7 & 16.1 & 37.8 & 2.0 & 8.0 & 29.4 \\
\hline HOMO-5 & -5.87 & 0.4 & 6.9 & 24.6 & 18.8 & 0.8 & 3.4 & 43.0 \\
\hline HOMO-6 & -5.97 & 0.1 & 10.8 & 21.0 & 1.6 & 0.4 & 0.2 & 65.4 \\
\hline
\end{tabular}


Table S2: Mulliken Analysis of the Contributions (\%) to the Molecular Orbitals of [6] and [11] from Analysis of the Alpha-Spin MOs from their DFT Calculations.

\begin{tabular}{|c|c|c|c|c|c|c|c|c|}
\hline \multirow{2}{*}{ Orbital } & \multirow{2}{*}{$\mathrm{E}(\mathrm{eV})$} & \multicolumn{3}{|c|}{$\mathrm{U}$} & \multicolumn{2}{|c|}{$\mathrm{C}=\mathrm{N}$} & \multirow{2}{*}{$\operatorname{Ar}_{\mathrm{F}}$} & \multirow{2}{*}{$\mathrm{C}_{5} \mathrm{Me}_{5}$} \\
\hline & & $\mathrm{sp}$ & d & $\mathrm{f}$ & $\mathrm{N}$ & $\mathrm{C}$ & & \\
\hline \multicolumn{9}{|l|}{$[6]^{-}$} \\
\hline $\mathrm{LUMO}+8$ & 3.84 & 22.2 & 11.2 & 17.2 & 2.7 & 2.0 & 4.2 & 36.4 \\
\hline LUMO+7 & 3.56 & 0.1 & 0.4 & 87.4 & 5.6 & 1.0 & 0.8 & 2.9 \\
\hline LUMO+6 & 3.37 & 0.0 & 7.0 & 65.1 & 0.2 & 1.0 & 13.9 & 11.6 \\
\hline LUMO+5 & 2.99 & 0.2 & 18.1 & 57.7 & 0.0 & 0.2 & 0.2 & 23.3 \\
\hline $\mathrm{LUMO}+4$ & 2.71 & 26.3 & 3.5 & 62.7 & 0.2 & 0.5 & 1.3 & 5.5 \\
\hline LUMO+3 & 2.54 & 0.1 & 0.2 & $9 \backslash 0.5$ & 0.5 & 0.0 & 97.6 & 0.6 \\
\hline LUMO+2 & 2.44 & 0.0 & 0.2 & 0.2 & 0.3 & 0.0 & 98.3 & 0.5 \\
\hline LUMO+1 & 2.16 & 0.1 & 2.8 & 23.6 & 14.4 & 4.3 & 49.2 & 4.6 \\
\hline LUMO & 2.00 & 0.4 & 6.1 & 16.2 & 17.0 & 8.5 & 46.3 & 4.1 \\
\hline HOMO & -0.12 & 0.1 & 2.9 & 63.8 & 3.4 & 14.0 & 12.1 & 2.1 \\
\hline HOMO-1 & -0.27 & 0.2 & 1.1 & 77.2 & 1.6 & 9.5 & 7.0 & 2.1 \\
\hline HOMO-2 & -0.60 & 0.1 & 3.4 & 92.8 & 0.5 & 0.0 & 0.0 & 2.5 \\
\hline HOMO-3 & -1.83 & 0.0 & 0.5 & 11.4 & 57.0 & 2.4 & 5.8 & 15.5 \\
\hline HOMO-4 & -2.09 & 2.0 & 2.8 & 2.2 & 11.1 & 0.9 & 1.3 & 78.5 \\
\hline HOMO-5 & -2.09 & 2.4 & 5.4 & 1.0 & 27.6 & 1.4 & 3.1 & 55.4 \\
\hline HOMO-6 & -2.35 & 0.2 & 7.0 & 5.9 & 2.8 & 1.1 & 1.6 & 80.7 \\
\hline \multicolumn{9}{|l|}{$[11]^{-}$} \\
\hline LUMO+8 & 3.18 & 0.1 & 5.2 & 17.7 & 3.8 & 6.2 & 60.2 & 5.0 \\
\hline LUMO+7 & 3.09 & 0.4 & 17.1 & 45.0 & 0.0 & 0.4 & 9.0 & 27.2 \\
\hline LUMO+6 & 3.07 & 0.4 & 10.0 & 21.2 & 9.4 & 12.4 & 36.8 & 7.8 \\
\hline LUMO+5 & 2.98 & 10.2 & 5.1 & 36.7 & 0.0 & 1.0 & 40.0 & 6.8 \\
\hline LUMO+4 & 2.71 & 21.4 & 2.5 & 19.1 & 0.8 & 1.4 & 51.0 & 3.2 \\
\hline LUMO+3 & 2.33 & 0.0 & 0.0 & 0.1 & 0.0 & 0.2 & 99.0 & 0.0 \\
\hline LUMO+2 & 2.32 & 0.0 & 0.1 & 0.1 & 0.0 & 0.2 & 99.0 & 0.0 \\
\hline LUMO+1 & 2.08 & 0.1 & 1.4 & 7.0 & 6.6 & 3.0 & 78.8 & 1.4 \\
\hline LUMO & 2.01 & 0.2 & 1.1 & 7.1 & 4.0 & 1.0 & 84.0 & 1.2 \\
\hline HOMO & -0.04 & 0.1 & 2.4 & 70.9 & 3.4 & 13.4 & 4.8 & 3.6 \\
\hline HOMO-1 & -0.11 & 0.1 & 1.0 & 80.4 & 2.2 & 10.0 & 2.4 & 2.4 \\
\hline HOMO-2 & -0.33 & 0.3 & 3.6 & 91.0 & 0.6 & 0.2 & 0.0 & 3.6 \\
\hline HOMO-3 & -1.92 & 0.4 & 0.1 & 8.4 & 31.4 & 1.4 & 4.0 & 50.4 \\
\hline HOMO-4 & -2.06 & 2.4 & 4.4 & 1.8 & 20.0 & 1.0 & 2.0 & 65.2 \\
\hline HOMO-5 & -2.18 & 1.7 & 2.5 & 3.7 & 32.4 & 2.0 & 5.8 & 49.0 \\
\hline HOMO-6 & -2.45 & 0.2 & 8.7 & 4.4 & 5.6 & 1.6 & 0.8 & 77.8 \\
\hline
\end{tabular}


Figure S1: Magnetic susceptibility data for $\left(\mathrm{C}_{5} \mathrm{Me}_{5}\right)_{2} \mathrm{U}\left[-\mathrm{N}=\mathrm{C}\left(\mathrm{CH}_{3}\right)\left(3-\mathrm{F}-\mathrm{C}_{6} \mathrm{H}_{4}\right)\right]_{2}$ (5) and $\left(\mathrm{C}_{5} \mathrm{Me}_{5}\right)_{2} \mathrm{U}\left[-\mathrm{N}=\mathrm{C}\left(\mathrm{CH}_{3}\right)\left(3,5-\mathrm{F}_{2}-\mathrm{C}_{6} \mathrm{H}_{3}\right)\right]_{2}(8)$ from 350 to $2 \mathrm{~K}$ at $5 \mathrm{~T}$. The small magnetic moments observed at low temperatures for $\mathbf{5}$ and $\mathbf{8}$ are consistent with the expected singlet ligand field ground states, however, small paramagnetic impurities reproducibly observed for both samples prevents observation of the LT temperature independent response for these complexes.

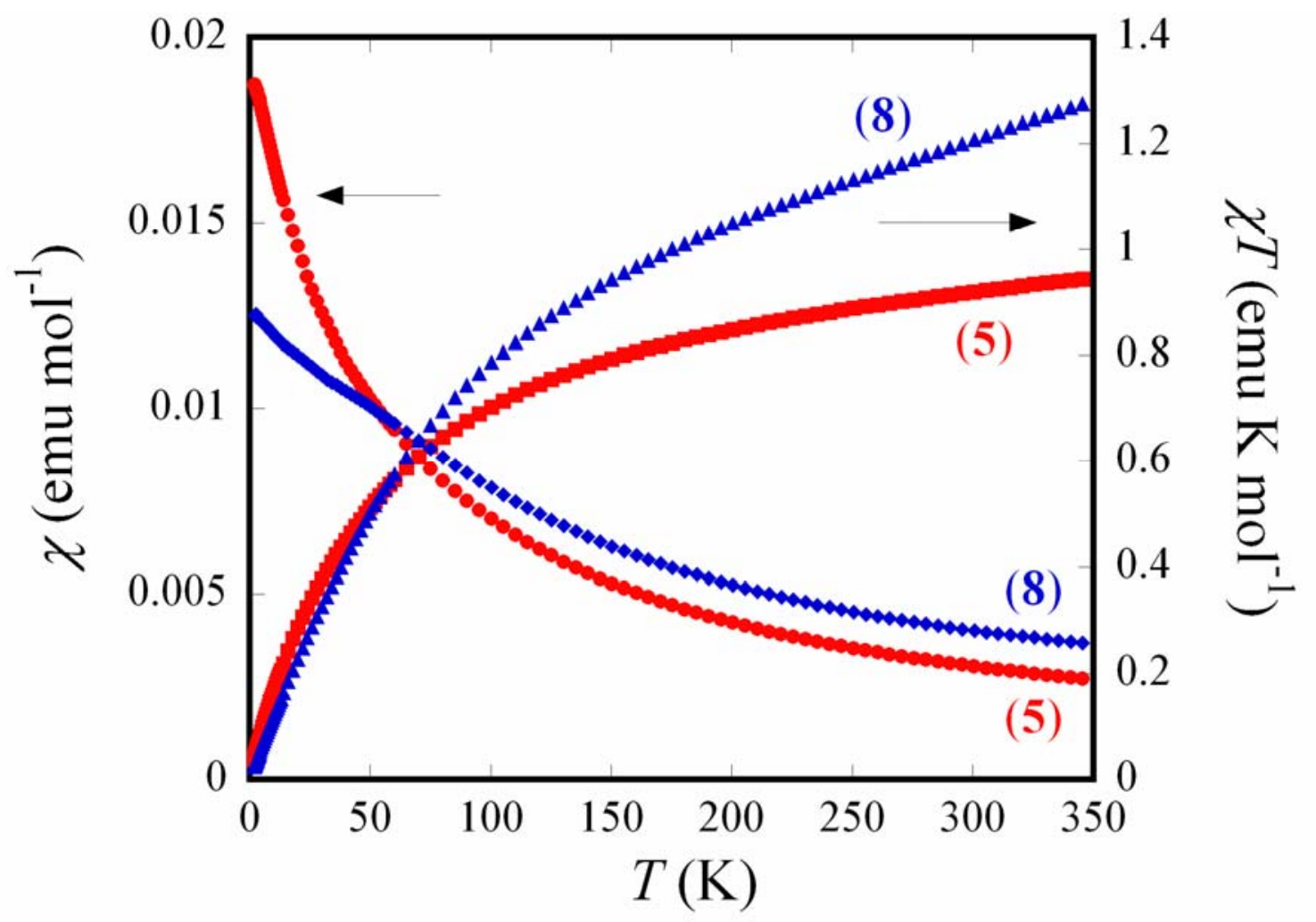


Figure S2: Thermal Ellipsoid Plot of $\left(\mathrm{C}_{5} \mathrm{Me}_{5}\right)_{2} \mathrm{U}\left[-\mathrm{N}=\mathrm{C}\left(\mathrm{CH}_{3}\right)\left(2-\mathrm{F}_{-} \mathrm{C}_{6} \mathrm{H}_{4}\right)\right]_{2}$ (4) with Ellipsoids Projected at the 50\% Probability Level.

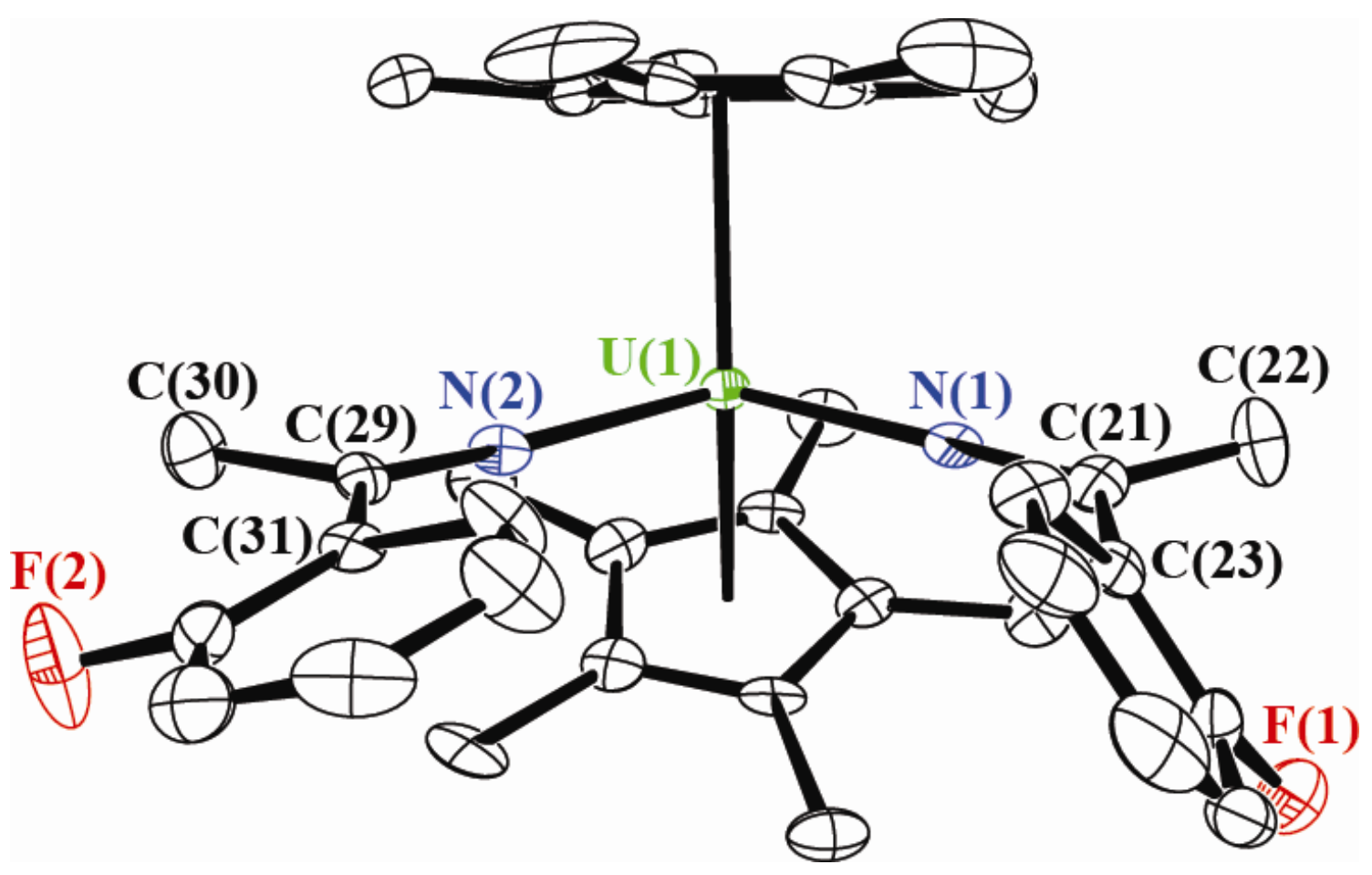


Table S3: Crystal Data and Structure Refinement for $\left(\mathrm{C}_{5} \mathrm{Me}_{5}\right)_{2} \mathrm{U}\left[-\mathrm{N}=\mathrm{C}\left(\mathrm{CH}_{3}\right)\left(2-\mathrm{F}-\mathrm{C}_{6} \mathrm{H}_{4}\right)\right]_{2}(4)$.

\begin{tabular}{|c|c|c|}
\hline Empirical formula & \multicolumn{2}{|l|}{$\mathrm{C}_{36} \mathrm{H}_{44} \mathrm{~F}_{2} \mathrm{~N}_{2} \mathrm{U}$} \\
\hline Formula weight & \multicolumn{2}{|l|}{780.76} \\
\hline Temperature & \multicolumn{2}{|l|}{$141(2) \mathrm{K}$} \\
\hline Wavelength & \multicolumn{2}{|l|}{$0.71073 \AA$} \\
\hline Crystal system & \multicolumn{2}{|l|}{ orthorhombic } \\
\hline Space group & \multicolumn{2}{|l|}{ P $2{ }_{1} 2_{1} 2_{1}$} \\
\hline \multirow[t]{3}{*}{ Unit cell dimensions } & $a=10.0173(5) \AA$ & $\alpha=90^{\circ}$ \\
\hline & $\mathrm{b}=17.6957(9) \AA$ & $\beta=90^{\circ}$ \\
\hline & $\mathrm{c}=18.322(1) \AA$ & $\gamma=90^{\circ}$ \\
\hline Volume & \multicolumn{2}{|l|}{$3247.8(3) \AA^{3}$} \\
\hline $\mathrm{Z}$ & \multicolumn{2}{|l|}{4} \\
\hline Density (calculated) & \multicolumn{2}{|l|}{$1.597 \mathrm{Mg} / \mathrm{m}^{3}$} \\
\hline Absorption coefficient & \multicolumn{2}{|l|}{$5.035 \mathrm{~mm}^{-1}$} \\
\hline $\mathrm{F}(000)$ & \multicolumn{2}{|l|}{1536} \\
\hline Crystal size & \multicolumn{2}{|c|}{$0.32 \times 0.14 \times 0.12 \mathrm{~mm}^{3}$} \\
\hline$\Theta$ Range for data collection & \multicolumn{2}{|l|}{1.60 to $29.00^{\circ}$} \\
\hline Index ranges & \multicolumn{2}{|c|}{$-13 \leq \mathrm{h} \leq 13,-23 \leq \mathrm{k} \leq 23,-24 \leq 1 \leq 24$} \\
\hline Reflections collected & \multicolumn{2}{|l|}{36595} \\
\hline Independent reflections & \multicolumn{2}{|c|}{$8028[\mathrm{R}(\mathrm{int})=0.0486]$} \\
\hline Completeness to $\Theta=25.00^{\circ}$ & \multicolumn{2}{|l|}{$100.0 \%$} \\
\hline Refinement method & \multicolumn{2}{|c|}{ Full-matrix least-squares on $\mathrm{F}^{2}$} \\
\hline Data / restraints / parameters & \multicolumn{2}{|l|}{$8028 / 0 / 370$} \\
\hline Goodness-of-fit on $\mathrm{F}^{2}$ & \multicolumn{2}{|l|}{1.339} \\
\hline Final $R$ indices $[\mathrm{I}>2 \sigma(\mathrm{I})]$ & \multicolumn{2}{|c|}{$\mathrm{R} 1=0.0316, \mathrm{wR} 2=0.0690$} \\
\hline $\mathrm{R}$ indices (all data) & \multicolumn{2}{|c|}{$\mathrm{R} 1=0.0353, \mathrm{wR} 2=0.0694$} \\
\hline Absolute structure parameter & \multicolumn{2}{|l|}{$0.021(7)$} \\
\hline Largest diff. peak and hole & \multicolumn{2}{|c|}{1.502 and -1.796 e. $\AA^{-3}$} \\
\hline
\end{tabular}


Table S4: Bond Distances $[\AA]$ and Angles $\left[{ }^{\circ}\right]$ for $\left(\mathrm{C}_{5} \mathrm{Me}_{5}\right)_{2} \mathrm{U}\left[-\mathrm{N}=\mathrm{C}\left(\mathrm{CH}_{3}\right)\left(2-\mathrm{F}_{-} \mathrm{C}_{6} \mathrm{H}_{4}\right)\right]_{2}$ (4).

\begin{tabular}{|c|c|c|c|}
\hline $\mathrm{U}(1)-\mathrm{N}(1)$ & $2.196(4)$ & $C(14)-C(19)$ & $1.487(8)$ \\
\hline $\mathrm{U}(1)-\mathrm{N}(2)$ & $2.200(4)$ & $C(15)-C(20)$ & $1.525(7)$ \\
\hline $\mathrm{U}(1)-\mathrm{C}(11)$ & $2.725(5)$ & $C(21)-C(23)$ & $1.505(8)$ \\
\hline $\mathrm{U}(1)-\mathrm{C}(15)$ & $2.731(5)$ & $C(21)-C(22)$ & $1.517(7)$ \\
\hline $\mathrm{U}(1)-\mathrm{C}(14)$ & $2.728(5)$ & $C(23)-C(24)$ & $1.373(8)$ \\
\hline $\mathrm{U}(1)-\mathrm{C}(2)$ & $2.737(5)$ & $C(23)-C(28)$ & $1.401(8)$ \\
\hline $\mathrm{U}(1)-\mathrm{C}(13)$ & $2.749(6)$ & $C(24)-C(25)$ & $1.386(10)$ \\
\hline $\mathrm{U}(1)-\mathrm{C}(5)$ & $2.738(5)$ & $C(25)-C(26)$ & $1.376(11)$ \\
\hline $\mathrm{U}(1)-\mathrm{C}(1)$ & $2.742(4)$ & $C(26)-C(27)$ & $1.385(9)$ \\
\hline $\mathrm{U}(1)-\mathrm{C}(3)$ & $2.744(5)$ & $\mathrm{C}(27)-\mathrm{C}(28)$ & $1.392(9)$ \\
\hline $\mathrm{U}(1)-\mathrm{C}(12)$ & $2.742(6)$ & $C(29)-C(31)$ & $1.525(7)$ \\
\hline $\mathrm{U}(1)-\mathrm{C}(4)$ & $2.776(6)$ & $C(29)-C(30)$ & $1.533(7)$ \\
\hline $\mathrm{F}(1)-\mathrm{C}(24)$ & $1.376(8)$ & $C(31)-C(32)$ & $1.372(8)$ \\
\hline $\mathrm{F}(2)-\mathrm{C}(32)$ & $1.345(7)$ & $C(31)-C(36)$ & $1.387(8)$ \\
\hline $\mathrm{N}(1)-\mathrm{C}(21)$ & $1.265(6)$ & $C(32)-C(33)$ & $1.394(9)$ \\
\hline $\mathrm{N}(2)-\mathrm{C}(29)$ & $1.245(6)$ & $\mathrm{C}(33)-\mathrm{C}(34)$ & $1.366(9)$ \\
\hline $\mathrm{C}(1)-\mathrm{C}(2)$ & $1.414(7)$ & $C(34)-C(35)$ & $1.355(9)$ \\
\hline$C(1)-C(5)$ & $1.428(7)$ & $C(35)-C(36)$ & $1.380(8)$ \\
\hline$C(1)-C(6)$ & $1.509(7)$ & & \\
\hline $\mathrm{C}(2)-\mathrm{C}(3)$ & $1.377(7)$ & $\mathrm{N}(1)-\mathrm{U}(1)-\mathrm{N}(2)$ & $109.92(15)$ \\
\hline$C(2)-C(7)$ & $1.511(7)$ & $\mathrm{N}(1)-\mathrm{U}(1)-\mathrm{C}(11)$ & $88.10(15)$ \\
\hline$C(3)-C(4)$ & $1.419(7)$ & $\mathrm{N}(2)-\mathrm{U}(1)-\mathrm{C}(11)$ & $126.64(15)$ \\
\hline$C(3)-C(8)$ & $1.536(7)$ & $\mathrm{N}(1)-\mathrm{U}(1)-\mathrm{C}(15)$ & $117.68(15)$ \\
\hline$C(4)-C(5)$ & $1.417(8)$ & $\mathrm{N}(2)-\mathrm{U}(1)-\mathrm{C}(15)$ & $110.61(15)$ \\
\hline$C(4)-C(9)$ & $1.502(8)$ & $C(11)-U(1)-C(15)$ & $29.59(15)$ \\
\hline$C(5)-C(10)$ & $1.525(7)$ & $\mathrm{N}(1)-\mathrm{U}(1)-\mathrm{C}(14)$ & $125.50(16)$ \\
\hline $\mathrm{C}(11)-\mathrm{C}(15)$ & $1.393(7)$ & $\mathrm{N}(2)-\mathrm{U}(1)-\mathrm{C}(14)$ & $81.81(16)$ \\
\hline $\mathrm{C}(11)-\mathrm{C}(12)$ & $1.423(7)$ & $C(11)-U(1)-C(14)$ & $49.29(17)$ \\
\hline$C(11)-C(16)$ & $1.509(7)$ & $C(15)-U(1)-C(14)$ & $29.55(15)$ \\
\hline $\mathrm{C}(12)-\mathrm{C}(13)$ & $1.381(8)$ & $\mathrm{N}(1)-\mathrm{U}(1)-\mathrm{C}(2)$ & $78.66(15)$ \\
\hline $\mathrm{C}(12)-\mathrm{C}(17)$ & $1.492(9)$ & $\mathrm{N}(2)-\mathrm{U}(1)-\mathrm{C}(2)$ & $122.01(15)$ \\
\hline$C(13)-C(14)$ & $1.452(8)$ & $\mathrm{C}(11)-\mathrm{U}(1)-\mathrm{C}(2)$ & $110.46(16)$ \\
\hline $\mathrm{C}(13)-\mathrm{C}(18)$ & $1.500(8)$ & $\mathrm{C}(15)-\mathrm{U}(1)-\mathrm{C}(2)$ & $114.66(15)$ \\
\hline$C(14)-C(15)$ & $1.392(7)$ & $C(14)-U(1)-C(2)$ & $140.67(17)$ \\
\hline
\end{tabular}




\begin{tabular}{|c|c|c|c|}
\hline $\mathrm{N}(1)-\mathrm{U}(1)-\mathrm{C}(13)$ & $97.54(18)$ & $C(2)-U(1)-C(12)$ & $132.67(18)$ \\
\hline $\mathrm{N}(2)-\mathrm{U}(1)-\mathrm{C}(13)$ & $78.27(16)$ & $\mathrm{C}(13)-\mathrm{U}(1)-\mathrm{C}(12)$ & $29.12(18)$ \\
\hline $\mathrm{C}(11)-\mathrm{U}(1)-\mathrm{C}(13)$ & $49.13(17)$ & $\mathrm{C}(5)-\mathrm{U}(1)-\mathrm{C}(12)$ & $145.22(17)$ \\
\hline $\mathrm{C}(15)-\mathrm{U}(1)-\mathrm{C}(13)$ & $49.23(16)$ & $\mathrm{C}(1)-\mathrm{U}(1)-\mathrm{C}(12)$ & $128.47(17)$ \\
\hline C(14)-U(1)-C(13) & $30.75(18)$ & $\mathrm{C}(3)-\mathrm{U}(1)-\mathrm{C}(12)$ & $154.59(18)$ \\
\hline $\mathrm{C}(2)-\mathrm{U}(1)-\mathrm{C}(13)$ & $159.56(17)$ & $\mathrm{N}(1)-\mathrm{U}(1)-\mathrm{C}(4)$ & $108.99(15)$ \\
\hline $\mathrm{N}(1)-\mathrm{U}(1)-\mathrm{C}(5)$ & $126.85(15)$ & $\mathrm{N}(2)-\mathrm{U}(1)-\mathrm{C}(4)$ & $75.81(15)$ \\
\hline $\mathrm{N}(2)-\mathrm{U}(1)-\mathrm{C}(5)$ & $92.00(16)$ & $\mathrm{C}(11)-\mathrm{U}(1)-\mathrm{C}(4)$ & $146.14(16)$ \\
\hline $\mathrm{C}(11)-\mathrm{U}(1)-\mathrm{C}(5)$ & $117.04(16)$ & $\mathrm{C}(15)-\mathrm{U}(1)-\mathrm{C}(4)$ & $125.48(16)$ \\
\hline $\mathrm{C}(15)-\mathrm{U}(1)-\mathrm{C}(5)$ & $96.56(15)$ & $\mathrm{C}(14)-\mathrm{U}(1)-\mathrm{C}(4)$ & $125.34(17)$ \\
\hline $\mathrm{C}(14)-\mathrm{U}(1)-\mathrm{C}(5)$ & $104.64(17)$ & $\mathrm{C}(2)-\mathrm{U}(1)-\mathrm{C}(4)$ & $48.84(15)$ \\
\hline $\mathrm{C}(2)-\mathrm{U}(1)-\mathrm{C}(5)$ & $49.41(15)$ & $\mathrm{C}(13)-\mathrm{U}(1)-\mathrm{C}(4)$ & $147.82(18)$ \\
\hline $\mathrm{C}(13)-\mathrm{U}(1)-\mathrm{C}(5)$ & $134.87(18)$ & $\mathrm{C}(5)-\mathrm{U}(1)-\mathrm{C}(4)$ & $29.77(16)$ \\
\hline $\mathrm{N}(1)-\mathrm{U}(1)-\mathrm{C}(1)$ & $105.76(14)$ & $\mathrm{C}(1)-\mathrm{U}(1)-\mathrm{C}(4)$ & $49.20(15)$ \\
\hline $\mathrm{N}(2)-\mathrm{U}(1)-\mathrm{C}(1)$ & $121.59(14)$ & $\mathrm{C}(3)-\mathrm{U}(1)-\mathrm{C}(4)$ & $29.79(15)$ \\
\hline $\mathrm{C}(11)-\mathrm{U}(1)-\mathrm{C}(1)$ & $98.71(16)$ & $\mathrm{C}(12)-\mathrm{U}(1)-\mathrm{C}(4)$ & $174.33(17)$ \\
\hline $\mathrm{C}(15)-\mathrm{U}(1)-\mathrm{C}(1)$ & $90.63(15)$ & $\mathrm{C}(21)-\mathrm{N}(1)-\mathrm{U}(1)$ & $174.1(4)$ \\
\hline $\mathrm{C}(14)-\mathrm{U}(1)-\mathrm{C}(1)$ & $112.06(17)$ & $\mathrm{C}(29)-\mathrm{N}(2)-\mathrm{U}(1)$ & $176.6(4)$ \\
\hline $\mathrm{C}(2)-\mathrm{U}(1)-\mathrm{C}(1)$ & 29.91(15) & $\mathrm{C}(2)-\mathrm{C}(1)-\mathrm{C}(5)$ & $107.3(4)$ \\
\hline $\mathrm{C}(13)-\mathrm{U}(1)-\mathrm{C}(1)$ & $139.75(18)$ & $\mathrm{C}(2)-\mathrm{C}(1)-\mathrm{C}(6)$ & $125.9(5)$ \\
\hline$C(5)-U(1)-C(1)$ & $30.20(15)$ & $\mathrm{C}(5)-\mathrm{C}(1)-\mathrm{C}(6)$ & $126.4(5)$ \\
\hline $\mathrm{N}(1)-\mathrm{U}(1)-\mathrm{C}(3)$ & $80.65(15)$ & $\mathrm{C}(2)-\mathrm{C}(1)-\mathrm{U}(1)$ & $74.8(3)$ \\
\hline $\mathrm{N}(2)-\mathrm{U}(1)-\mathrm{C}(3)$ & $93.75(16)$ & $\mathrm{C}(5)-\mathrm{C}(1)-\mathrm{U}(1)$ & $74.7(3)$ \\
\hline $\mathrm{C}(11)-\mathrm{U}(1)-\mathrm{C}(3)$ & $139.40(16)$ & $\mathrm{C}(6)-\mathrm{C}(1)-\mathrm{U}(1)$ & $121.9(3)$ \\
\hline $\mathrm{C}(15)-\mathrm{U}(1)-\mathrm{C}(3)$ & $139.48(15)$ & $\mathrm{C}(3)-\mathrm{C}(2)-\mathrm{C}(1)$ & $108.7(5)$ \\
\hline $\mathrm{C}(14)-\mathrm{U}(1)-\mathrm{C}(3)$ & $153.49(17)$ & $\mathrm{C}(3)-\mathrm{C}(2)-\mathrm{C}(7)$ & $126.6(5)$ \\
\hline $\mathrm{C}(2)-\mathrm{U}(1)-\mathrm{C}(3)$ & $29.11(15)$ & $\mathrm{C}(1)-\mathrm{C}(2)-\mathrm{C}(7)$ & $124.1(5)$ \\
\hline $\mathrm{C}(13)-\mathrm{U}(1)-\mathrm{C}(3)$ & $170.74(15)$ & $\mathrm{C}(3)-\mathrm{C}(2)-\mathrm{U}(1)$ & $75.7(3)$ \\
\hline $\mathrm{C}(5)-\mathrm{U}(1)-\mathrm{C}(3)$ & $49.17(15)$ & $\mathrm{C}(1)-\mathrm{C}(2)-\mathrm{U}(1)$ & $75.3(3)$ \\
\hline $\mathrm{C}(1)-\mathrm{U}(1)-\mathrm{C}(3)$ & $48.86(16)$ & $\mathrm{C}(7)-\mathrm{C}(2)-\mathrm{U}(1)$ & $122.3(3)$ \\
\hline $\mathrm{N}(1)-\mathrm{U}(1)-\mathrm{C}(12)$ & $76.43(16)$ & $\mathrm{C}(2)-\mathrm{C}(3)-\mathrm{C}(4)$ & $109.2(5)$ \\
\hline $\mathrm{N}(2)-\mathrm{U}(1)-\mathrm{C}(12)$ & $104.19(17)$ & $\mathrm{C}(2)-\mathrm{C}(3)-\mathrm{C}(8)$ & $126.7(5)$ \\
\hline $\mathrm{C}(11)-\mathrm{U}(1)-\mathrm{C}(12)$ & $30.16(16)$ & $\mathrm{C}(4)-\mathrm{C}(3)-\mathrm{C}(8)$ & $123.8(5)$ \\
\hline $\mathrm{C}(15)-\mathrm{U}(1)-\mathrm{C}(12)$ & $49.05(16)$ & $\mathrm{C}(2)-\mathrm{C}(3)-\mathrm{U}(1)$ & $75.2(3)$ \\
\hline $\mathrm{C}(14)-\mathrm{U}(1)-\mathrm{C}(12)$ & $49.43(17)$ & $\mathrm{C}(4)-\mathrm{C}(3)-\mathrm{U}(1)$ & $76.4(3)$ \\
\hline
\end{tabular}




\begin{tabular}{|c|c|c|c|}
\hline $\mathrm{C}(8)-\mathrm{C}(3)-\mathrm{U}(1)$ & $120.1(3)$ & $\mathrm{C}(13)-\mathrm{C}(14)-\mathrm{U}(1)$ & $75.4(3)$ \\
\hline$C(5)-C(4)-C(3)$ & $107.1(5)$ & $\mathrm{C}(19)-\mathrm{C}(14)-\mathrm{U}(1)$ & $121.9(4)$ \\
\hline$C(5)-C(4)-C(9)$ & $126.1(5)$ & $C(14)-C(15)-C(11)$ & $109.5(4)$ \\
\hline$C(3)-C(4)-C(9)$ & $126.8(5)$ & $C(14)-C(15)-C(20)$ & $124.2(5)$ \\
\hline$C(5)-C(4)-U(1)$ & $73.6(3)$ & $C(11)-C(15)-C(20)$ & $125.5(5)$ \\
\hline$C(3)-C(4)-U(1)$ & $73.9(3)$ & $\mathrm{C}(14)-\mathrm{C}(15)-\mathrm{U}(1)$ & $75.1(3)$ \\
\hline$C(9)-C(4)-U(1)$ & $120.5(4)$ & $\mathrm{C}(11)-\mathrm{C}(15)-\mathrm{U}(1)$ & $75.0(3)$ \\
\hline$C(4)-C(5)-C(1)$ & $107.7(4)$ & $\mathrm{C}(20)-\mathrm{C}(15)-\mathrm{U}(1)$ & $124.4(3)$ \\
\hline$C(4)-C(5)-C(10)$ & $126.0(5)$ & $\mathrm{N}(1)-\mathrm{C}(21)-\mathrm{C}(23)$ & $120.3(5)$ \\
\hline$C(1)-C(5)-C(10)$ & $126.1(5)$ & $\mathrm{N}(1)-\mathrm{C}(21)-\mathrm{C}(22)$ & $123.5(5)$ \\
\hline$C(4)-C(5)-U(1)$ & $76.6(3)$ & $C(23)-C(21)-C(22)$ & $116.0(5)$ \\
\hline$C(1)-C(5)-U(1)$ & 75.1(3) & $\mathrm{C}(24)-\mathrm{C}(23)-\mathrm{C}(28)$ & $115.5(6)$ \\
\hline $\mathrm{C}(10)-\mathrm{C}(5)-\mathrm{U}(1)$ & $117.8(3)$ & $\mathrm{C}(24)-\mathrm{C}(23)-\mathrm{C}(21)$ & $123.5(5)$ \\
\hline$C(15)-C(11)-C(12)$ & $107.6(5)$ & $\mathrm{C}(28)-\mathrm{C}(23)-\mathrm{C}(21)$ & $121.0(5)$ \\
\hline$C(15)-C(11)-C(16)$ & $127.8(5)$ & $\mathrm{C}(23)-\mathrm{C}(24)-\mathrm{C}(25)$ & $123.7(6)$ \\
\hline$C(12)-C(11)-C(16)$ & $124.6(5)$ & $\mathrm{C}(23)-\mathrm{C}(24)-\mathrm{F}(1)$ & $118.3(6)$ \\
\hline $\mathrm{C}(15)-\mathrm{C}(11)-\mathrm{U}(1)$ & 75.4(3) & $\mathrm{C}(25)-\mathrm{C}(24)-\mathrm{F}(1)$ & $118.0(6)$ \\
\hline $\mathrm{C}(12)-\mathrm{C}(11)-\mathrm{U}(1)$ & $75.6(3)$ & $\mathrm{C}(24)-\mathrm{C}(25)-\mathrm{C}(26)$ & 119.6(6) \\
\hline $\mathrm{C}(16)-\mathrm{C}(11)-\mathrm{U}(1)$ & $116.9(3)$ & $\mathrm{C}(27)-\mathrm{C}(26)-\mathrm{C}(25)$ & $118.9(7)$ \\
\hline $\mathrm{C}(13)-\mathrm{C}(12)-\mathrm{C}(11)$ & $108.6(6)$ & $\mathrm{C}(26)-\mathrm{C}(27)-\mathrm{C}(28)$ & $120.0(7)$ \\
\hline$C(13)-C(12)-C(17)$ & $126.7(7)$ & $\mathrm{C}(27)-\mathrm{C}(28)-\mathrm{C}(23)$ & $122.1(6)$ \\
\hline $\mathrm{C}(11)-\mathrm{C}(12)-\mathrm{C}(17)$ & $124.4(6)$ & $\mathrm{N}(2)-\mathrm{C}(29)-\mathrm{C}(31)$ & $121.0(4)$ \\
\hline $\mathrm{C}(13)-\mathrm{C}(12)-\mathrm{U}(1)$ & $75.7(4)$ & $\mathrm{N}(2)-\mathrm{C}(29)-\mathrm{C}(30)$ & $121.4(5)$ \\
\hline $\mathrm{C}(11)-\mathrm{C}(12)-\mathrm{U}(1)$ & $74.3(3)$ & $\mathrm{C}(31)-\mathrm{C}(29)-\mathrm{C}(30)$ & $117.5(4)$ \\
\hline $\mathrm{C}(17)-\mathrm{C}(12)-\mathrm{U}(1)$ & $121.2(4)$ & $\mathrm{C}(32)-\mathrm{C}(31)-\mathrm{C}(36)$ & $115.8(5)$ \\
\hline$C(12)-C(13)-C(14)$ & $107.7(6)$ & $\mathrm{C}(32)-\mathrm{C}(31)-\mathrm{C}(29)$ & $125.3(5)$ \\
\hline $\mathrm{C}(12)-\mathrm{C}(13)-\mathrm{C}(18)$ & $125.7(7)$ & $\mathrm{C}(36)-\mathrm{C}(31)-\mathrm{C}(29)$ & $118.9(5)$ \\
\hline $\mathrm{C}(14)-\mathrm{C}(13)-\mathrm{C}(18)$ & $126.2(6)$ & $\mathrm{F}(2)-\mathrm{C}(32)-\mathrm{C}(31)$ & $120.7(6)$ \\
\hline $\mathrm{C}(12)-\mathrm{C}(13)-\mathrm{U}(1)$ & $75.2(4)$ & $\mathrm{F}(2)-\mathrm{C}(32)-\mathrm{C}(33)$ & 116.1(6) \\
\hline $\mathrm{C}(14)-\mathrm{C}(13)-\mathrm{U}(1)$ & $73.8(3)$ & $\mathrm{C}(31)-\mathrm{C}(32)-\mathrm{C}(33)$ & $123.2(6)$ \\
\hline $\mathrm{C}(18)-\mathrm{C}(13)-\mathrm{U}(1)$ & $122.9(4)$ & $\mathrm{C}(34)-\mathrm{C}(33)-\mathrm{C}(32)$ & $118.0(6)$ \\
\hline$C(15)-C(14)-C(13)$ & $106.7(5)$ & $\mathrm{C}(35)-\mathrm{C}(34)-\mathrm{C}(33)$ & 121.1(6) \\
\hline$C(15)-C(14)-C(19)$ & $127.4(6)$ & $\mathrm{C}(34)-\mathrm{C}(35)-\mathrm{C}(36)$ & $119.4(6)$ \\
\hline$C(13)-C(14)-C(19)$ & $125.3(6)$ & $\mathrm{C}(35)-\mathrm{C}(36)-\mathrm{C}(31)$ & $122.3(6)$ \\
\hline $\mathrm{C}(15)-\mathrm{C}(14)-\mathrm{U}(1)$ & $75.3(3)$ & & \\
\hline
\end{tabular}


Figure S3: Thermal ellipsoid plot of one of the two disordered components in the structure of $\left(\mathrm{C}_{5} \mathrm{Me}_{5}\right)_{2} \mathrm{U}\left[-\mathrm{N}=\mathrm{C}\left(\mathrm{CH}_{3}\right)\left(4-\mathrm{F}-\mathrm{C}_{6} \mathrm{H}_{4}\right)\right]_{2}(6)$ with ellipsoids projected at the $50 \%$ probability level. The $\mathrm{U}(1)$ atom lies on a crystallographically imposed inversion center.

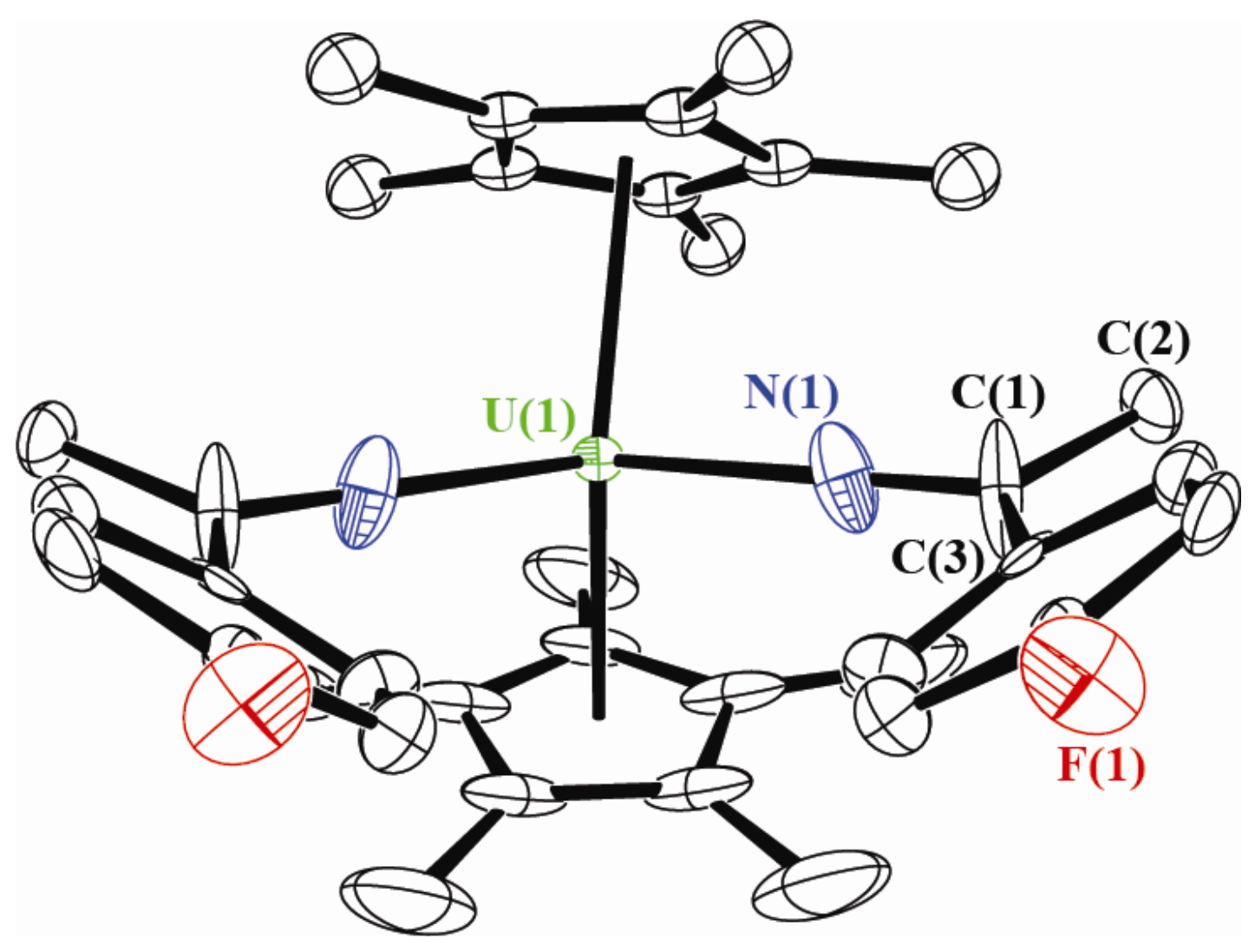


Table S5: Crystal Data and Structure Refinement for $\left(\mathrm{C}_{5} \mathrm{Me}_{5}\right)_{2} \mathrm{U}\left[-\mathrm{N}=\mathrm{C}\left(\mathrm{CH}_{3}\right)\left(4-\mathrm{F}-\mathrm{C}_{6} \mathrm{H}_{4}\right)\right]_{2}(6)$.

Empirical formula

Formula weight

Temperature

Wavelength

Crystal system

Space group

Unit cell dimensions

Volume

Z

Density (calculated)

Absorption coefficient

$\mathrm{F}(000)$

Crystal size

$\Theta$ Range for data collection

Index ranges

Reflections collected

Independent reflections

Completeness to $\Theta=25.00^{\circ}$

Max. and min. transmission

Refinement method

Data / restraints / parameters

Goodness-of-fit on $\mathrm{F}^{2}$

Final $R$ indices $[\mathrm{I}>2 \sigma(\mathrm{I})]$

$\mathrm{R}$ indices (all data)

Largest diff. peak and hole
$\mathrm{C}_{36} \mathrm{H}_{44} \mathrm{~F}_{2} \mathrm{~N}_{2} \mathrm{U}$

780.76

141(1) K

$0.71073 \AA$

orthorhombic

P nma

$\mathrm{a}=17.9790(19) \AA$

$\alpha=90^{\circ}$

$\mathrm{b}=18.0889(19) \AA$

$\beta=90^{\circ}$

$\mathrm{c}=9.9044(11) \AA$

$\gamma=90^{\circ}$

4

$1.610 \mathrm{Mg} / \mathrm{m}^{3}$

$5.077 \mathrm{~mm}^{-1}$

1536

$0.20 \times 0.20 \times 0.20 \mathrm{~mm}^{3}$

2.25 to $28.87^{\circ}$

$-23 \leq \mathrm{h} \leq 23,-24 \leq \mathrm{k} \leq 23,-13 \leq 1 \leq 13$

34385

$4194[\mathrm{R}($ int $)=0.0736]$

$100.0 \%$

0.4300 and 0.4300

Full-matrix least-squares on $\mathrm{F}^{2}$

4194 / 0 / 137

3.568

$\mathrm{R} 1=0.0735, \mathrm{wR} 2=0.1954$

$\mathrm{R} 1=0.0804, \mathrm{wR} 2=0.1960$

3.782 and -8.327 e. $\AA^{-3}$ 
Table S6: Bond Distances $[\AA]$ and Angles $\left[{ }^{\circ}\right]$ for $\left(\mathrm{C}_{5} \mathrm{Me}_{5}\right)_{2} \mathrm{U}\left[-\mathrm{N}=\mathrm{C}\left(\mathrm{CH}_{3}\right)\left(4-\mathrm{F}-\mathrm{C}_{6} \mathrm{H}_{4}\right)\right]_{2}(6)$.

\begin{tabular}{|c|c|c|c|}
\hline $\mathrm{U}(1)-\mathrm{N}(1) \# 1$ & $2.169(9)$ & $C(6)-C\left(5^{\prime}\right)$ & 1.1324 \\
\hline $\mathrm{U}(1)-\mathrm{N}(1)$ & $2.169(9)$ & $C(6)-C(7)$ & 1.3900 \\
\hline $\mathrm{U}(1)-\mathrm{C}(10)$ & $2.712(10)$ & $C(7)-C\left(6^{\prime}\right)$ & 0.7258 \\
\hline $\mathrm{U}(1)-\mathrm{C}(10) \# 1$ & $2.712(10)$ & $C(7)-C\left(7^{\prime}\right)$ & 0.7298 \\
\hline $\mathrm{U}(1)-\mathrm{C}(11)$ & $2.715(16)$ & $C(7)-C(8)$ & 1.3900 \\
\hline $\mathrm{U}(1)-\mathrm{C}(17) \# 1$ & $2.756(6)$ & $C(7)-C\left(5^{\prime}\right)$ & 1.8088 \\
\hline $\mathrm{U}(1)-\mathrm{C}(17)$ & $2.756(7)$ & $C(7)-C\left(8^{\prime}\right)$ & 1.8119 \\
\hline $\mathrm{U}(1)-\mathrm{C}(9)$ & $2.756(10)$ & $\mathrm{C}(8)-\mathrm{C}\left(8^{\prime}\right)$ & 0.5733 \\
\hline $\mathrm{U}(1)-\mathrm{C}(9) \# 1$ & $2.756(10)$ & $C(8)-C\left(7^{\prime}\right)$ & 1.1861 \\
\hline $\mathrm{U}(1)-\mathrm{C}(16)$ & $2.777(7)$ & $\mathrm{C}(8)-\mathrm{C}\left(3^{\prime}\right)$ & 1.2679 \\
\hline $\mathrm{U}(1)-\mathrm{C}(18)$ & $2.778(7)$ & $C\left(3^{\prime}\right)-C\left(4^{\prime}\right)$ & 1.3900 \\
\hline $\mathrm{U}(1)-\mathrm{C}(15)$ & $2.813(8)$ & $\mathrm{C}\left(3^{\prime}\right)-\mathrm{C}\left(8^{\prime}\right)$ & 1.3900 \\
\hline $\mathrm{F}(1)-\mathrm{F}\left(1^{\prime}\right)$ & $1.000(18)$ & $C\left(4^{\prime}\right)-C\left(5^{\prime}\right)$ & 1.3900 \\
\hline $\mathrm{F}(1)-\mathrm{C}(6)$ & $1.321(14)$ & $\mathrm{C}\left(5^{\prime}\right)-\mathrm{C}\left(6^{\prime}\right)$ & 1.3900 \\
\hline $\mathrm{F}(1)-\mathrm{C}\left(6^{\prime}\right)$ & $1.739(16)$ & $C\left(6^{\prime}\right)-C\left(7^{\prime}\right)$ & 1.3900 \\
\hline $\mathrm{F}\left(1^{\prime}\right)-\mathrm{C}\left(6^{\prime}\right)$ & $1.318(14)$ & $\mathrm{C}\left(7^{\prime}\right)-\mathrm{C}\left(8^{\prime}\right)$ & 1.3900 \\
\hline $\mathrm{F}\left(1^{\prime}\right)-\mathrm{C}(6)$ & $1.488(16)$ & $C(9)-C(10)$ & $1.426(15)$ \\
\hline $\mathrm{N}(1)-\mathrm{C}(1)$ & $1.289(13)$ & $\mathrm{C}(9)-\mathrm{C}(9) \# 1$ & $1.45(3)$ \\
\hline$C(1)-C\left(3^{\prime}\right)$ & $1.434(12)$ & $C(9)-C(12)$ & $1.49(2)$ \\
\hline$C(1)-C\left(2^{\prime}\right)$ & $1.57(2)$ & $C(10)-C(11)$ & $1.410(16)$ \\
\hline $\mathrm{C}(1)-\mathrm{C}(3)$ & $1.591(13)$ & $C(10)-C(13)$ & $1.54(2)$ \\
\hline $\mathrm{C}(1)-\mathrm{C}(2)$ & $1.64(2)$ & $\mathrm{C}(11)-\mathrm{C}(10) \# 1$ & $1.410(16)$ \\
\hline $\mathrm{C}(2)-\mathrm{C}\left(2^{\prime}\right)$ & $0.83(3)$ & $C(11)-C(14)$ & $1.52(3)$ \\
\hline $\mathrm{C}(3)-\mathrm{C}\left(3^{\prime}\right)$ & 0.6111 & $\mathrm{C}(15)-\mathrm{C}(18) \# 1$ & $1.135(12)$ \\
\hline$C(3)-C\left(4^{\prime}\right)$ & 1.1770 & $\mathrm{C}(15)-\mathrm{C}(17) \# 1$ & $1.193(12)$ \\
\hline $\mathrm{C}(3)-\mathrm{C}(4)$ & 1.3900 & $C(15)-C(19)$ & 1.4200 \\
\hline $\mathrm{C}(3)-\mathrm{C}(8)$ & 1.3900 & $\mathrm{C}(15)-\mathrm{C}(16)$ & 1.4200 \\
\hline $\mathrm{C}(3)-\mathrm{C}\left(8^{\prime}\right)$ & 1.6736 & $C(15)-C(20)$ & 1.5095 \\
\hline$C(4)-C\left(4^{\prime}\right)$ & 0.9293 & $\mathrm{C}(15)-\mathrm{C}(23) \# 1$ & $1.625(4)$ \\
\hline$C(4)-C(5)$ & 1.3900 & $\mathrm{C}(15)-\mathrm{C}(22) \# 1$ & $1.717(3)$ \\
\hline$C(4)-C\left(5^{\prime}\right)$ & 1.6839 & $\mathrm{C}(16)-\mathrm{C}(17) \# 1$ & $0.452(3)$ \\
\hline$C(5)-C\left(5^{\prime}\right)$ & 1.0316 & $\mathrm{C}(16)-\mathrm{C}(22) \# 1$ & $1.244(17)$ \\
\hline$C(5)-C(6)$ & 1.3900 & $\mathrm{C}(16)-\mathrm{C}(17)$ & 1.4200 \\
\hline$C(5)-C\left(4^{\prime}\right)$ & 1.7091 & $\mathrm{C}(16)-\mathrm{C}(16) \# 1$ & $1.440(17)$ \\
\hline
\end{tabular}




\begin{tabular}{|c|c|c|c|}
\hline $\mathrm{C}(6)-\mathrm{C}\left(6^{\prime}\right)$ & 0.8820 & $C(16)-C(21)$ & 1.5095 \\
\hline$C(16)-C(18) \# 1$ & 1.8446 & $\mathrm{~N}(1) \# 1-\mathrm{U}(1)-\mathrm{C}(17) \# 1$ & $120.5(3)$ \\
\hline$C(17)-C(16) \# 1$ & $0.452(3)$ & $\mathrm{N}(1)-\mathrm{U}(1)-\mathrm{C}(17) \# 1$ & $97.4(3)$ \\
\hline $\mathrm{C}(17)-\mathrm{C}(15) \# 1$ & $1.193(13)$ & $\mathrm{C}(10)-\mathrm{U}(1)-\mathrm{C}(17) \# 1$ & $107.7(3)$ \\
\hline $\mathrm{C}(17)-\mathrm{C}(17) \# 1$ & $1.259(16)$ & $\mathrm{C}(10) \# 1-\mathrm{U}(1)-\mathrm{C}(17) \# 1$ & 119.7(3) \\
\hline $\mathrm{C}(17)-\mathrm{C}(18)$ & 1.4200 & $\mathrm{C}(11)-\mathrm{U}(1)-\mathrm{C}(17) \# 1$ & $98.9(4)$ \\
\hline $\mathrm{C}(17)-\mathrm{C}(22)$ & 1.5095 & N(1)\#1-U(1)-C(17) & $97.4(3)$ \\
\hline$C(18)-C(19) \# 1$ & $0.4838(11)$ & $\mathrm{N}(1)-\mathrm{U}(1)-\mathrm{C}(17)$ & $120.5(3)$ \\
\hline$C(18)-C(15) \# 1$ & $1.135(13)$ & $\mathrm{C}(10)-\mathrm{U}(1)-\mathrm{C}(17)$ & $119.7(3)$ \\
\hline $\mathrm{C}(18)-\mathrm{C}(18) \# 1$ & $1.303(15)$ & $\mathrm{C}(10) \# 1-\mathrm{U}(1)-\mathrm{C}(17)$ & $107.7(3)$ \\
\hline $\mathrm{C}(18)-\mathrm{C}(19)$ & 1.4200 & $\mathrm{C}(11)-\mathrm{U}(1)-\mathrm{C}(17)$ & $98.9(4)$ \\
\hline $\mathrm{C}(18)-\mathrm{C}(23)$ & 1.5095 & $\mathrm{C}(17) \# 1-\mathrm{U}(1)-\mathrm{C}(17)$ & $26.4(3)$ \\
\hline$C(18)-C(16) \# 1$ & 1.8446 & N(1)\#1-U(1)-C(9) & $100.8(4)$ \\
\hline$C(19)-C(18) \# 1$ & $0.4838(11)$ & $\mathrm{N}(1)-\mathrm{U}(1)-\mathrm{C}(9)$ & $75.6(4)$ \\
\hline$C(19)-C(23) \# 1$ & $1.294(16)$ & $\mathrm{C}(10)-\mathrm{U}(1)-\mathrm{C}(9)$ & $30.2(3)$ \\
\hline$C(19)-C(19) \# 1$ & $1.367(16)$ & $\mathrm{C}(10) \# 1-\mathrm{U}(1)-\mathrm{C}(9)$ & $50.0(4)$ \\
\hline C(19)-C(24) & 1.5095 & $\mathrm{C}(11)-\mathrm{U}(1)-\mathrm{C}(9)$ & $50.1(4)$ \\
\hline$C(21)-C(22) \# 1$ & $1.175(10)$ & $\mathrm{C}(17) \# 1-\mathrm{U}(1)-\mathrm{C}(9)$ & $137.0(3)$ \\
\hline$C(22)-C(21) \# 1$ & $1.175(12)$ & $\mathrm{C}(17)-\mathrm{U}(1)-\mathrm{C}(9)$ & $148.4(3)$ \\
\hline$C(22)-C(16) \# 1$ & $1.244(15)$ & $\mathrm{N}(1) \# 1-\mathrm{U}(1)-\mathrm{C}(9) \# 1$ & $75.6(4)$ \\
\hline$C(22)-C(15) \# 1$ & $1.717(10)$ & $\mathrm{N}(1)-\mathrm{U}(1)-\mathrm{C}(9) \# 1$ & $100.8(4)$ \\
\hline$C(23)-C(19) \# 1$ & $1.294(15)$ & $\mathrm{C}(10)-\mathrm{U}(1)-\mathrm{C}(9) \# 1$ & $50.0(4)$ \\
\hline$C(23)-C(24) \# 1$ & $1.322(12)$ & $\mathrm{C}(10) \# 1-\mathrm{U}(1)-\mathrm{C}(9) \# 1$ & $30.2(3)$ \\
\hline$C(23)-C(15) \# 1$ & $1.625(10)$ & $\mathrm{C}(11)-\mathrm{U}(1)-\mathrm{C}(9) \# 1$ & $50.1(4)$ \\
\hline \multirow[t]{2}{*}{$\mathrm{C}(24)-\mathrm{C}(23) \# 1$} & $1.322(10)$ & $\mathrm{C}(17) \# 1-\mathrm{U}(1)-\mathrm{C}(9) \# 1$ & $148.4(3)$ \\
\hline & & $\mathrm{C}(17)-\mathrm{U}(1)-\mathrm{C}(9) \# 1$ & $137.0(3)$ \\
\hline $\mathrm{N}(1) \# 1-\mathrm{U}(1)-\mathrm{N}(1)$ & $112.0(5)$ & $\mathrm{C}(9)-\mathrm{U}(1)-\mathrm{C}(9) \# 1$ & $30.5(6)$ \\
\hline N(1)\#1-U(1)-C(10) & $125.5(4)$ & $\mathrm{N}(1) \# 1-\mathrm{U}(1)-\mathrm{C}(16)$ & $126.7(3)$ \\
\hline $\mathrm{N}(1)-\mathrm{U}(1)-\mathrm{C}(10)$ & $83.4(4)$ & $\mathrm{N}(1)-\mathrm{U}(1)-\mathrm{C}(16)$ & $99.7(3)$ \\
\hline $\mathrm{N}(1) \# 1-\mathrm{U}(1)-\mathrm{C}(10) \# 1$ & $83.4(4)$ & $\mathrm{C}(10)-\mathrm{U}(1)-\mathrm{C}(16)$ & $98.8(3)$ \\
\hline $\mathrm{N}(1)-\mathrm{U}(1)-\mathrm{C}(10) \# 1$ & $125.5(4)$ & $\mathrm{C}(10) \# 1-\mathrm{U}(1)-\mathrm{C}(16)$ & 111.7(3) \\
\hline $\mathrm{C}(10)-\mathrm{U}(1)-\mathrm{C}(10) \# 1$ & $49.6(6)$ & $\mathrm{C}(11)-\mathrm{U}(1)-\mathrm{C}(16)$ & $89.7(4)$ \\
\hline $\mathrm{N}(1) \# 1-\mathrm{U}(1)-\mathrm{C}(11)$ & 113.3(3) & $\mathrm{C}(17) \# 1-\mathrm{U}(1)-\mathrm{C}(16)$ & $9.36(7)$ \\
\hline $\mathrm{N}(1)-\mathrm{U}(1)-\mathrm{C}(11)$ & $113.3(3)$ & $\mathrm{C}(17)-\mathrm{U}(1)-\mathrm{C}(16)$ & 29.74(7) \\
\hline $\mathrm{C}(10)-\mathrm{U}(1)-\mathrm{C}(11)$ & $30.1(3)$ & $\mathrm{C}(9)-\mathrm{U}(1)-\mathrm{C}(16)$ & $128.6(3)$ \\
\hline
\end{tabular}




\begin{tabular}{|c|c|c|c|}
\hline $\mathrm{C}(10) \# 1-\mathrm{U}(1)-\mathrm{C}(11)$ & $30.1(3)$ & $\mathrm{C}(9) \# 1-\mathrm{U}(1)-\mathrm{C}(16)$ & $139.5(3)$ \\
\hline $\mathrm{N}(1) \# 1-\mathrm{U}(1)-\mathrm{C}(18)$ & $81.6(3)$ & & \\
\hline $\mathrm{N}(1)-\mathrm{U}(1)-\mathrm{C}(18)$ & 104.1(3) & $C\left(3^{\prime}\right)-C(1)-C(2)$ & $114.9(11)$ \\
\hline $\mathrm{C}(10)-\mathrm{U}(1)-\mathrm{C}(18)$ & $147.4(3)$ & $C\left(2^{\prime}\right)-C(1)-C(2)$ & $30.0(10)$ \\
\hline $\mathrm{C}(10) \# 1-\mathrm{U}(1)-\mathrm{C}(18)$ & $130.2(3)$ & $C(3)-C(1)-C(2)$ & $127.7(11)$ \\
\hline $\mathrm{C}(11)-\mathrm{U}(1)-\mathrm{C}(18)$ & $128.4(4)$ & $C\left(2^{\prime}\right)-C(2)-C(1)$ & $71(2)$ \\
\hline $\mathrm{C}(17) \# 1-\mathrm{U}(1)-\mathrm{C}(18)$ & $40.4(2)$ & $C(2)-C\left(2^{\prime}\right)-C(1)$ & $79(2)$ \\
\hline $\mathrm{C}(17)-\mathrm{U}(1)-\mathrm{C}(18)$ & $29.74(7)$ & $C\left(3^{\prime}\right)-C(3)-C\left(4^{\prime}\right)$ & 96.9 \\
\hline $\mathrm{C}(9)-\mathrm{U}(1)-\mathrm{C}(18)$ & $177.5(3)$ & $C\left(3^{\prime}\right)-C(3)-C(4)$ & 137.6 \\
\hline $\mathrm{C}(9) \# 1-\mathrm{U}(1)-\mathrm{C}(18)$ & $151.1(3)$ & $C\left(4^{\prime}\right)-C(3)-C(4)$ & 41.4 \\
\hline $\mathrm{C}(16)-\mathrm{U}(1)-\mathrm{C}(18)$ & $48.86(10)$ & $\mathrm{C}\left(3^{\prime}\right)-\mathrm{C}(3)-\mathrm{C}(8)$ & 65.7 \\
\hline $\mathrm{N}(1) \# 1-\mathrm{U}(1)-\mathrm{C}(15)$ & $127.5(3)$ & $\mathrm{C}\left(4^{\prime}\right)-\mathrm{C}(3)-\mathrm{C}(8)$ & 101.4 \\
\hline $\mathrm{N}(1)-\mathrm{U}(1)-\mathrm{C}(15)$ & $72.9(3)$ & $\mathrm{C}(4)-\mathrm{C}(3)-\mathrm{C}(8)$ & 120.0 \\
\hline $\mathrm{C}(10)-\mathrm{U}(1)-\mathrm{C}(15)$ & $107.0(3)$ & $\mathrm{C}\left(3^{\prime}\right)-\mathrm{C}(3)-\mathrm{C}(1)$ & $64.1(8)$ \\
\hline $\mathrm{C}(10) \# 1-\mathrm{U}(1)-\mathrm{C}(15)$ & $138.5(3)$ & $C\left(4^{\prime}\right)-C(3)-C(1)$ & $118.8(7)$ \\
\hline $\mathrm{C}(11)-\mathrm{U}(1)-\mathrm{C}(15)$ & $111.1(3)$ & $\mathrm{C}(4)-\mathrm{C}(3)-\mathrm{C}(1)$ & $121.6(6)$ \\
\hline $\mathrm{C}(17) \# 1-\mathrm{U}(1)-\mathrm{C}(15)$ & $24.7(2)$ & $C(8)-C(3)-C(1)$ & $117.8(7)$ \\
\hline C(17)-U(1)-C(15) & $48.73(10)$ & $\mathrm{C}\left(3^{\prime}\right)-\mathrm{C}(3)-\mathrm{C}\left(8^{\prime}\right)$ & 52.6 \\
\hline $\mathrm{C}(9)-\mathrm{U}(1)-\mathrm{C}(15)$ & $129.3(3)$ & $C\left(4^{\prime}\right)-C(3)-C\left(8^{\prime}\right)$ & 114.1 \\
\hline $\mathrm{C}(9) \# 1-\mathrm{U}(1)-\mathrm{C}(15)$ & $156.9(3)$ & $\mathrm{C}(4)-\mathrm{C}(3)-\mathrm{C}\left(8^{\prime}\right)$ & 138.7 \\
\hline $\mathrm{C}(16)-\mathrm{U}(1)-\mathrm{C}(15)$ & $29.42(7)$ & $\mathrm{C}(8)-\mathrm{C}(3)-\mathrm{C}\left(8^{\prime}\right)$ & 18.8 \\
\hline $\mathrm{C}(18)-\mathrm{U}(1)-\mathrm{C}(15)$ & $48.53(9)$ & $\mathrm{C}(1)-\mathrm{C}(3)-\mathrm{C}\left(8^{\prime}\right)$ & $99.0(7)$ \\
\hline$F\left(1^{\prime}\right)-F(1)-C(6)$ & $78.4(13)$ & $C\left(4^{\prime}\right)-C(4)-C(5)$ & 92.8 \\
\hline $\mathrm{F}\left(1^{\prime}\right)-\mathrm{F}(1)-\mathrm{C}\left(6^{\prime}\right)$ & $48.9(10)$ & $C\left(4^{\prime}\right)-C(4)-C(3)$ & 56.9 \\
\hline $\mathrm{C}(6)-\mathrm{F}(1)-\mathrm{C}\left(6^{\prime}\right)$ & $29.7(4)$ & $C(5)-C(4)-C(3)$ & 120.0 \\
\hline $\mathrm{F}(1)-\mathrm{F}\left(1^{\prime}\right)-\mathrm{C}\left(6^{\prime}\right)$ & $96.3(14)$ & $C\left(4^{\prime}\right)-C(4)-C\left(5^{\prime}\right)$ & 55.6 \\
\hline $\mathrm{F}(1)-\mathrm{F}\left(1^{\prime}\right)-\mathrm{C}(6)$ & $60.4(11)$ & $C(5)-C(4)-C\left(5^{\prime}\right)$ & 37.7 \\
\hline$C\left(6^{\prime}\right)-\mathrm{F}\left(1^{\prime}\right)-\mathrm{C}(6)$ & $36.0(4)$ & $\mathrm{C}(3)-\mathrm{C}(4)-\mathrm{C}\left(5^{\prime}\right)$ & 90.7 \\
\hline $\mathrm{C}(1)-\mathrm{N}(1)-\mathrm{U}(1)$ & $179.0(8)$ & $C\left(5^{\prime}\right)-C(5)-C(4)$ & 86.8 \\
\hline $\mathrm{N}(1)-\mathrm{C}(1)-\mathrm{C}\left(3^{\prime}\right)$ & $124.1(10)$ & $C\left(5^{\prime}\right)-C(5)-C(6)$ & 53.3 \\
\hline $\mathrm{N}(1)-\mathrm{C}(1)-\mathrm{C}\left(2^{\prime}\right)$ & $126.4(12)$ & $C(4)-C(5)-C(6)$ & 120.0 \\
\hline $\mathrm{C}\left(3^{\prime}\right)-\mathrm{C}(1)-\mathrm{C}\left(2^{\prime}\right)$ & $109.3(11)$ & $C\left(5^{\prime}\right)-C(5)-C\left(4^{\prime}\right)$ & 54.4 \\
\hline $\mathrm{N}(1)-\mathrm{C}(1)-\mathrm{C}(3)$ & 119.9(9) & $C(4)-C(5)-C\left(4^{\prime}\right)$ & 32.9 \\
\hline$C\left(3^{\prime}\right)-C(1)-C(3)$ & $22.5(2)$ & $C(6)-C(5)-C\left(4^{\prime}\right)$ & 97.8 \\
\hline \multirow[t]{2}{*}{$\mathrm{C}\left(2^{\prime}\right)-\mathrm{C}(1)-\mathrm{C}(3)$} & $110.7(12)$ & $\mathrm{C}\left(6^{\prime}\right)-\mathrm{C}(6)-\mathrm{C}\left(5^{\prime}\right)$ & 86.3 \\
\hline & & $C\left(6^{\prime}\right)-C(6)-F(1)$ & $102.4(9)$ \\
\hline
\end{tabular}




\begin{tabular}{|c|c|c|c|}
\hline $\mathrm{N}(1)-\mathrm{C}(1)-\mathrm{C}(2)$ & $1117(12)$ & $\mathrm{C}\left(5^{\prime}\right)-\mathrm{C}(6)-\mathrm{F}(1)$ & $131.7(10)$ \\
\hline $\mathrm{C}\left(6^{\prime}\right)-\mathrm{C}(6)-\mathrm{C}(7)$ & 27.1 & $\mathrm{C}\left(3^{\prime}\right)-\mathrm{C}(8)-\mathrm{C}(3)$ & 26.1 \\
\hline $\mathrm{C}\left(5^{\prime}\right)-\mathrm{C}(6)-\mathrm{C}(7)$ & 91.0 & $C(7)-C(8)-C(3)$ & 120.0 \\
\hline $\mathrm{F}(1)-\mathrm{C}(6)-\mathrm{C}(7)$ & $117.9(9)$ & $C(3)-C\left(3^{\prime}\right)-C(8)$ & 88.2 \\
\hline $\mathrm{C}\left(6^{\prime}\right)-\mathrm{C}(6)-\mathrm{C}(5)$ & 129.4 & $\mathrm{C}(3)-\mathrm{C}\left(3^{\prime}\right)-\mathrm{C}\left(4^{\prime}\right)$ & 57.2 \\
\hline $\mathrm{C}\left(5^{\prime}\right)-\mathrm{C}(6)-\mathrm{C}(5)$ & 469 & $\mathrm{C}(8)-\mathrm{C}\left(3^{\prime}\right)-\mathrm{C}\left(4^{\prime}\right)$ & 96.9 \\
\hline $\mathrm{F}(1)-\mathrm{C}(6)-\mathrm{C}(5)$ & 1221(9) & $\mathrm{C}(3)-\mathrm{C}\left(3^{\prime}\right)-\mathrm{C}\left(8^{\prime}\right)$ & 107.0 \\
\hline $\mathrm{C}(7)-\mathrm{C}(6)-\mathrm{C}(5)$ & 120.0 & $\mathrm{C}(8)-\mathrm{C}\left(3^{\prime}\right)-\mathrm{C}\left(8^{\prime}\right)$ & 24.4 \\
\hline$C\left(6^{\prime}\right)-C(6)-F\left(1^{\prime}\right)$ & $61.4(8)$ & $\mathrm{C}\left(4^{\prime}\right)-\mathrm{C}\left(3^{\prime}\right)-\mathrm{C}\left(8^{\prime}\right)$ & 120.0 \\
\hline $\mathrm{C}\left(5^{\prime}\right)-\mathrm{C}(6)-\mathrm{F}\left(1^{\prime}\right)$ & $127.1(8)$ & $C(3)-C\left(3^{\prime}\right)-C(1)$ & 93.3(9) \\
\hline $\mathrm{F}(1)-\mathrm{C}(6)-\mathrm{F}\left(1^{\prime}\right)$ & $41.2(8)$ & $\mathrm{C}(8)-\mathrm{C}\left(3^{\prime}\right)-\mathrm{C}(1)$ & $141.9(7)$ \\
\hline $\mathrm{C}(7)-\mathrm{C}(6)-\mathrm{F}\left(1^{\prime}\right)$ & $78.4(8)$ & $C\left(4^{\prime}\right)-C\left(3^{\prime}\right)-C(1)$ & $115.8(7)$ \\
\hline $\mathrm{C}(5)-\mathrm{C}(6)-\mathrm{F}\left(1^{\prime}\right)$ & $157.8(8)$ & $\mathrm{C}\left(8^{\prime}\right)-\mathrm{C}\left(3^{\prime}\right)-\mathrm{C}(1)$ & 123.1(7) \\
\hline$C\left(6^{\prime}\right)-C(7)-C\left(7^{\prime}\right)$ & 145.5 & $C(4)-C\left(4^{\prime}\right)-C(3)$ & 81.7 \\
\hline $\mathrm{C}\left(6^{\prime}\right)-\mathrm{C}(7)-\mathrm{C}(6)$ & 33.6 & $\mathrm{C}(4)-\mathrm{C}\left(4^{\prime}\right)-\mathrm{C}\left(3^{\prime}\right)$ & 107.2 \\
\hline$C\left(7^{\prime}\right)-C(7)-C(6)$ & 177.7 & $C(3)-C\left(4^{\prime}\right)-C\left(3^{\prime}\right)$ & 25.9 \\
\hline $\mathrm{C}\left(6^{\prime}\right)-\mathrm{C}(7)-\mathrm{C}(8)$ & 130.3 & $\mathrm{C}(4)-\mathrm{C}\left(4^{\prime}\right)-\mathrm{C}\left(5^{\prime}\right)$ & 90.9 \\
\hline $\mathrm{C}\left(7^{\prime}\right)-\mathrm{C}(7)-\mathrm{C}(8)$ & 58.6 & $C(3)-C\left(4^{\prime}\right)-C\left(5^{\prime}\right)$ & 117.4 \\
\hline $\mathrm{C}(6)-\mathrm{C}(7)-\mathrm{C}(8)$ & 120.0 & $\mathrm{C}\left(3^{\prime}\right)-\mathrm{C}\left(4^{\prime}\right)-\mathrm{C}\left(5^{\prime}\right)$ & 120.0 \\
\hline$C\left(6^{\prime}\right)-C(7)-C\left(5^{\prime}\right)$ & 44.7 & $C(4)-C\left(4^{\prime}\right)-C(5)$ & 54.3 \\
\hline $\mathrm{C}\left(7^{\prime}\right)-\mathrm{C}(7)-\mathrm{C}\left(5^{\prime}\right)$ & 139.0 & $C(3)-C\left(4^{\prime}\right)-C(5)$ & 111.7 \\
\hline $\mathrm{C}(6)-\mathrm{C}(7)-\mathrm{C}\left(5^{\prime}\right)$ & 388 & $C\left(3^{\prime}\right)-C\left(4^{\prime}\right)-C(5)$ & 131.2 \\
\hline $\mathrm{C}(8)-\mathrm{C}(7)-\mathrm{C}\left(5^{\prime}\right)$ & 88.2 & $C\left(5^{\prime}\right)-C\left(4^{\prime}\right)-C(5)$ & 37.1 \\
\hline $\mathrm{C}\left(6^{\prime}\right)-\mathrm{C}(7)-\mathrm{C}\left(8^{\prime}\right)$ & 139.0 & $C(5)-C\left(5^{\prime}\right)-C(6)$ & 79.8 \\
\hline $\mathrm{C}\left(7^{\prime}\right)-\mathrm{C}(7)-\mathrm{C}\left(8^{\prime}\right)$ & 44.6 & $\mathrm{C}(5)-\mathrm{C}\left(5^{\prime}\right)-\mathrm{C}\left(6^{\prime}\right)$ & 116.3 \\
\hline $\mathrm{C}(6)-\mathrm{C}(7)-\mathrm{C}\left(8^{\prime}\right)$ & 133.9 & $\mathrm{C}(6)-\mathrm{C}\left(5^{\prime}\right)-\mathrm{C}\left(6^{\prime}\right)$ & 39.3 \\
\hline $\mathrm{C}(8)-\mathrm{C}(7)-\mathrm{C}\left(8^{\prime}\right)$ & 14.0 & $C(5)-C\left(5^{\prime}\right)-C\left(4^{\prime}\right)$ & 88.5 \\
\hline $\mathrm{C}\left(5^{\prime}\right)-\mathrm{C}(7)-\mathrm{C}\left(8^{\prime}\right)$ & 100.3 & $C(6)-C\left(5^{\prime}\right)-C\left(4^{\prime}\right)$ & 136.6 \\
\hline $\mathrm{C}\left(8^{\prime}\right)-\mathrm{C}(8)-\mathrm{C}\left(7^{\prime}\right)$ & 98.3 & $C\left(6^{\prime}\right)-C\left(5^{\prime}\right)-C\left(4^{\prime}\right)$ & 120.0 \\
\hline $\mathrm{C}\left(8^{\prime}\right)-\mathrm{C}(8)-\mathrm{C}\left(3^{\prime}\right)$ & 89.8 & $C(5)-C\left(5^{\prime}\right)-C(4)$ & 55.5 \\
\hline $\mathrm{C}\left(7^{\prime}\right)-\mathrm{C}(8)-\mathrm{C}\left(3^{\prime}\right)$ & 157.7 & $C(6)-C\left(5^{\prime}\right)-C(4)$ & 116.1 \\
\hline $\mathrm{C}\left(8^{\prime}\right)-\mathrm{C}(8)-\mathrm{C}(7)$ & 129.9 & $C\left(6^{\prime}\right)-C\left(5^{\prime}\right)-C(4)$ & 125.8 \\
\hline $\mathrm{C}\left(7^{\prime}\right)-\mathrm{C}(8)-\mathrm{C}(7)$ & 31.7 & $C\left(4^{\prime}\right)-C\left(5^{\prime}\right)-C(4)$ & 33.5 \\
\hline $\mathrm{C}\left(3^{\prime}\right)-\mathrm{C}(8)-\mathrm{C}(7)$ & 134.9 & $C(5)-C\left(5^{\prime}\right)-C(7)$ & 113.0 \\
\hline $\mathrm{C}\left(8^{\prime}\right)-\mathrm{C}(8)-\mathrm{C}(3)$ & 109.8 & $\begin{array}{l}\mathrm{C}(6)-\mathrm{C}\left(5^{\prime}\right)-\mathrm{C}(7) \\
\mathrm{C}\left(6^{\prime}\right)-\mathrm{C}\left(5^{\prime}\right)-\mathrm{C}(7)\end{array}$ & $\begin{array}{l}50.2 \\
21.5\end{array}$ \\
\hline
\end{tabular}




\begin{tabular}{|c|c|c|c|}
\hline$C\left(7^{\prime}\right)-C(8)-C(3)$ & 151.6 & $\mathrm{C}(9) \# 1-\mathrm{C}(9)-\mathrm{C}(12)$ & $125.3(8)$ \\
\hline$C\left(4^{\prime}\right)-C\left(5^{\prime}\right)-C(7)$ & 99.4 & $\mathrm{C}(10)-\mathrm{C}(9)-\mathrm{U}(1)$ & $73.2(6)$ \\
\hline$C(4)-C\left(5^{\prime}\right)-C(7)$ & 105.4 & $\mathrm{C}(9) \# 1-\mathrm{C}(9)-\mathrm{U}(1)$ & $74.7(3)$ \\
\hline$C(7)-C\left(6^{\prime}\right)-C(6)$ & 119.3 & $\mathrm{C}(12)-\mathrm{C}(9)-\mathrm{U}(1)$ & $122.1(9)$ \\
\hline$C(7)-C\left(6^{\prime}\right)-F\left(1^{\prime}\right)$ & 123.3(9) & $C(11)-C(10)-C(9)$ & $109.5(12)$ \\
\hline$C(6)-C\left(6^{\prime}\right)-F\left(1^{\prime}\right)$ & $82.6(9)$ & $\mathrm{C}(11)-\mathrm{C}(10)-\mathrm{C}(13)$ & $127.4(12)$ \\
\hline$C(7)-C\left(6^{\prime}\right)-C\left(7^{\prime}\right)$ & 17.3 & $C(9)-C(10)-C(13)$ & $123.0(13)$ \\
\hline $\mathrm{C}(6)-\mathrm{C}\left(6^{\prime}\right)-\mathrm{C}\left(7^{\prime}\right)$ & 136.6 & $\mathrm{C}(11)-\mathrm{C}(10)-\mathrm{U}(1)$ & $75.1(7)$ \\
\hline $\mathrm{F}\left(1^{\prime}\right)-\mathrm{C}\left(6^{\prime}\right)-\mathrm{C}\left(7^{\prime}\right)$ & $119.5(9)$ & $\mathrm{C}(9)-\mathrm{C}(10)-\mathrm{U}(1)$ & $76.6(6)$ \\
\hline$C(7)-C\left(6^{\prime}\right)-C\left(5^{\prime}\right)$ & 113.8 & $\mathrm{C}(13)-\mathrm{C}(10)-\mathrm{U}(1)$ & $118.7(9)$ \\
\hline$C(6)-C\left(6^{\prime}\right)-C\left(5^{\prime}\right)$ & 54.4 & $\mathrm{C}(10)-\mathrm{C}(11)-\mathrm{C}(10) \# 1$ & $107.5(15)$ \\
\hline $\mathrm{F}\left(1^{\prime}\right)-\mathrm{C}\left(6^{\prime}\right)-\mathrm{C}\left(5^{\prime}\right)$ & $120.5(9)$ & $C(10)-C(11)-C(14)$ & $125.9(7)$ \\
\hline$C\left(7^{\prime}\right)-C\left(6^{\prime}\right)-C\left(5^{\prime}\right)$ & 120.0 & C(10)\#1-C(11)-C(14) & $125.9(7)$ \\
\hline$C(7)-C\left(6^{\prime}\right)-F(1)$ & $137.0(8)$ & $C(10)-C(11)-U(1)$ & $74.8(8)$ \\
\hline$C(6)-C\left(6^{\prime}\right)-F(1)$ & $47.9(7)$ & $\mathrm{C}(10) \# 1-\mathrm{C}(11)-\mathrm{U}(1)$ & $74.8(8)$ \\
\hline $\mathrm{F}\left(1^{\prime}\right)-\mathrm{C}\left(6^{\prime}\right)-\mathrm{F}(1)$ & $34.9(7)$ & $\mathrm{C}(14)-\mathrm{C}(11)-\mathrm{U}(1)$ & $124.2(12)$ \\
\hline$C\left(7^{\prime}\right)-C\left(6^{\prime}\right)-F(1)$ & $144.7(7)$ & $\mathrm{C}(18) \# 1-\mathrm{C}(15)-\mathrm{C}(17) \# 1$ & 75.1(9) \\
\hline$C\left(5^{\prime}\right)-C\left(6^{\prime}\right)-F(1)$ & $90.7(7)$ & $C(18) \# 1-C(15)-C(19)$ & $17.7(4)$ \\
\hline$C(7)-C\left(7^{\prime}\right)-C(8)$ & 89.8 & $\mathrm{C}(17) \# 1-\mathrm{C}(15)-\mathrm{C}(19)$ & $92.0(5)$ \\
\hline$C(7)-C\left(7^{\prime}\right)-C\left(8^{\prime}\right)$ & 113.8 & $C(18) \# 1-C(15)-C(16)$ & $91.8(5)$ \\
\hline $\mathrm{C}(8)-\mathrm{C}\left(7^{\prime}\right)-\mathrm{C}\left(8^{\prime}\right)$ & 24.1 & $C(17) \# 1-C(15)-C(16)$ & $17.3(4)$ \\
\hline$C(7)-C\left(7^{\prime}\right)-C\left(6^{\prime}\right)$ & 17.2 & $C(19)-C(15)-C(16)$ & 108.0 \\
\hline$C(8)-C\left(7^{\prime}\right)-C\left(6^{\prime}\right)$ & 97.4 & $C(18) \# 1-C(15)-C(20)$ & $141.6(5)$ \\
\hline$C\left(8^{\prime}\right)-C\left(7^{\prime}\right)-C\left(6^{\prime}\right)$ & 120.0 & $C(17) \# 1-C(15)-C(20)$ & $141.5(5)$ \\
\hline $\mathrm{C}(8)-\mathrm{C}\left(8^{\prime}\right)-\mathrm{C}\left(7^{\prime}\right)$ & 57.6 & $C(19)-C(15)-C(20)$ & 126.0 \\
\hline $\mathrm{C}(8)-\mathrm{C}\left(8^{\prime}\right)-\mathrm{C}\left(3^{\prime}\right)$ & 65.8 & $C(16)-C(15)-C(20)$ & 126.0 \\
\hline $\mathrm{C}\left(7^{\prime}\right)-\mathrm{C}\left(8^{\prime}\right)-\mathrm{C}\left(3^{\prime}\right)$ & 120.0 & $\mathrm{C}(18) \# 1-\mathrm{C}(15)-\mathrm{C}(23) \# 1$ & $63.4(2)$ \\
\hline $\mathrm{C}(8)-\mathrm{C}\left(8^{\prime}\right)-\mathrm{C}(3)$ & 51.4 & $\mathrm{C}(17) \# 1-\mathrm{C}(15)-\mathrm{C}(23) \# 1$ & $135.1(8)$ \\
\hline$C\left(7^{\prime}\right)-C\left(8^{\prime}\right)-C(3)$ & 108.9 & $C(19)-C(15)-C(23) \# 1$ & $49.7(6)$ \\
\hline$C\left(3^{\prime}\right)-C\left(8^{\prime}\right)-C(3)$ & 20.4 & $C(16)-C(15)-C(23) \# 1$ & $152.2(5)$ \\
\hline $\mathrm{C}(8)-\mathrm{C}\left(8^{\prime}\right)-\mathrm{C}(7)$ & 36.1 & $C(20)-C(15)-C(23) \# 1$ & $78.3(6)$ \\
\hline$C\left(7^{\prime}\right)-C\left(8^{\prime}\right)-C(7)$ & 21.6 & $\mathrm{C}(18) \# 1-\mathrm{C}(15)-\mathrm{C}(22) \# 1$ & $131.4(8)$ \\
\hline$C\left(3^{\prime}\right)-C\left(8^{\prime}\right)-C(7)$ & 99.3 & $\mathrm{C}(17) \# 1-\mathrm{C}(15)-\mathrm{C}(22) \# 1$ & $59.3(2)$ \\
\hline$C(3)-C\left(8^{\prime}\right)-C(7)$ & 87.3 & $C(19)-C(15)-C(22) \# 1$ & $149.1(5)$ \\
\hline $\mathrm{C}(10)-\mathrm{C}(9)-\mathrm{C}(9) \# 1$ & $106.8(8)$ & $\mathrm{C}(16)-\mathrm{C}(15)-\mathrm{C}(22) \# 1$ & 5(6) \\
\hline
\end{tabular}




\begin{tabular}{|c|c|c|c|}
\hline$C(10)-C(9)-C(12)$ & $127.7(13)$ & $\mathrm{C}(21)-\mathrm{C}(16)-\mathrm{U}(1)$ & $115.2(2)$ \\
\hline$C(20)-C(15)-C(22) \# 1$ & $82.3(6)$ & $\mathrm{C}(18) \# 1-\mathrm{C}(16)-\mathrm{U}(1)$ & $70.6(2)$ \\
\hline $\mathrm{C}(23) \# 1-\mathrm{C}(15)-\mathrm{C}(22) \# 1$ & $145.7(7)$ & $C(16) \# 1-C(17)-C(15) \# 1$ & 111(2) \\
\hline $\mathrm{C}(18) \# 1-\mathrm{C}(15)-\mathrm{U}(1)$ & $76.6(4)$ & $\mathrm{C}(16) \# 1-\mathrm{C}(17)-\mathrm{C}(17) \# 1$ & $102(2)$ \\
\hline $\mathrm{C}(17) \# 1-\mathrm{C}(15)-\mathrm{U}(1)$ & $75.0(4)$ & $\mathrm{C}(15) \# 1-\mathrm{C}(17)-\mathrm{C}(17) \# 1$ & 139.1(6) \\
\hline $\mathrm{C}(19)-\mathrm{C}(15)-\mathrm{U}(1)$ & $75.4(2)$ & $\mathrm{C}(16) \# 1-\mathrm{C}(17)-\mathrm{C}(16)$ & $83(2)$ \\
\hline $\mathrm{C}(16)-\mathrm{C}(15)-\mathrm{U}(1)$ & $73.9(3)$ & C(15)\#1-C(17)-C(16) & $151.8(7)$ \\
\hline $\mathrm{C}(20)-\mathrm{C}(15)-\mathrm{U}(1)$ & $116.8(3)$ & $\mathrm{C}(17) \# 1-\mathrm{C}(17)-\mathrm{C}(16)$ & 18.2 \\
\hline $\mathrm{C}(23) \# 1-\mathrm{C}(15)-\mathrm{U}(1)$ & 109.3(4) & C(16)\#1-C(17)-C(18) & 157.1(11) \\
\hline $\mathrm{C}(22) \# 1-\mathrm{C}(15)-\mathrm{U}(1)$ & $104.6(4)$ & $C(15) \# 1-C(17)-C(18)$ & $50.6(6)$ \\
\hline $\mathrm{C}(17) \# 1-\mathrm{C}(16)-\mathrm{C}(22) \# 1$ & $118(2)$ & C(17)\#1-C(17)-C(18) & 90.9 \\
\hline $\mathrm{C}(17) \# 1-\mathrm{C}(16)-\mathrm{C}(17)$ & $60(2)$ & $C(16)-C(17)-C(18)$ & 108.0 \\
\hline $\mathrm{C}(22) \# 1-\mathrm{C}(16)-\mathrm{C}(17)$ & $160.0(3)$ & $\mathrm{C}(16) \# 1-\mathrm{C}(17)-\mathrm{C}(22)$ & $46.7(19)$ \\
\hline$C(17) \# 1-C(16)-C(15)$ & $51.6(18)$ & $C(15) \# 1-C(17)-C(22)$ & $77.9(6)$ \\
\hline $\mathrm{C}(22) \# 1-\mathrm{C}(16)-\mathrm{C}(15)$ & $80.0(4)$ & $\mathrm{C}(17) \# 1-\mathrm{C}(17)-\mathrm{C}(22)$ & 142.6 \\
\hline$C(17)-C(16)-C(15)$ & 108.0 & $C(16)-C(17)-C(22)$ & 126.0 \\
\hline $\mathrm{C}(17) \# 1-\mathrm{C}(16)-\mathrm{C}(16) \# 1$ & $78(2)$ & $\mathrm{C}(18)-\mathrm{C}(17)-\mathrm{C}(22)$ & 126.0 \\
\hline $\mathrm{C}(22) \# 1-\mathrm{C}(16)-\mathrm{C}(16) \# 1$ & $153.1(4)$ & $\mathrm{C}(16) \# 1-\mathrm{C}(17)-\mathrm{U}(1)$ & $88.1(5)$ \\
\hline $\mathrm{C}(17)-\mathrm{C}(16)-\mathrm{C}(16) \# 1$ & 18.2 & $\mathrm{C}(15) \# 1-\mathrm{C}(17)-\mathrm{U}(1)$ & $80.3(5)$ \\
\hline$C(15)-C(16)-C(16) \# 1$ & 124.9 & $\mathrm{C}(17) \# 1-\mathrm{C}(17)-\mathrm{U}(1)$ & $76.8(3)$ \\
\hline $\mathrm{C}(17) \# 1-\mathrm{C}(16)-\mathrm{C}(21)$ & $161.8(9)$ & $\mathrm{C}(16)-\mathrm{C}(17)-\mathrm{U}(1)$ & $76.0(3)$ \\
\hline $\mathrm{C}(22) \# 1-\mathrm{C}(16)-\mathrm{C}(21)$ & $49.4(3)$ & $\mathrm{C}(18)-\mathrm{C}(17)-\mathrm{U}(1)$ & $76.0(2)$ \\
\hline$C(17)-C(16)-C(21)$ & 126.0 & $\mathrm{C}(22)-\mathrm{C}(17)-\mathrm{U}(1)$ & 114.3(3) \\
\hline$C(15)-C(16)-C(21)$ & 126.0 & $\mathrm{C}(19) \# 1-\mathrm{C}(18)-\mathrm{C}(15) \# 1$ & $117(2)$ \\
\hline$C(16) \# 1-C(16)-C(21)$ & 108.8 & C(19)\#1-C(18)-C(18)\#1 & $93.8(19)$ \\
\hline $\mathrm{C}(17) \# 1-\mathrm{C}(16)-\mathrm{C}(18) \# 1$ & $17.5(8)$ & $\mathrm{C}(15) \# 1-\mathrm{C}(18)-\mathrm{C}(18) \# 1$ & $140.8(6)$ \\
\hline $\mathrm{C}(22) \# 1-\mathrm{C}(16)-\mathrm{C}(18) \# 1$ & $114.0(7)$ & $\mathrm{C}(19) \# 1-\mathrm{C}(18)-\mathrm{C}(19)$ & $73.9(19)$ \\
\hline $\mathrm{C}(17)-\mathrm{C}(16)-\mathrm{C}(18) \# 1$ & $70.3(5)$ & $\mathrm{C}(15) \# 1-\mathrm{C}(18)-\mathrm{C}(19)$ & $154.2(7)$ \\
\hline$C(15)-C(16)-C(18) \# 1$ & $37.9(5)$ & $\mathrm{C}(18) \# 1-\mathrm{C}(18)-\mathrm{C}(19)$ & 19.9 \\
\hline $\mathrm{C}(16) \# 1-\mathrm{C}(16)-\mathrm{C}(18) \# 1$ & $87.9(5)$ & $\mathrm{C}(19) \# 1-\mathrm{C}(18)-\mathrm{C}(17)$ & $162.0(3)$ \\
\hline $\mathrm{C}(21)-\mathrm{C}(16)-\mathrm{C}(18) \# 1$ & $163.1(5)$ & $\mathrm{C}(15) \# 1-\mathrm{C}(18)-\mathrm{C}(17)$ & $54.3(6)$ \\
\hline $\mathrm{C}(17) \# 1-\mathrm{C}(16)-\mathrm{U}(1)$ & $82.6(5)$ & $\mathrm{C}(18) \# 1-\mathrm{C}(18)-\mathrm{C}(17)$ & 89.1 \\
\hline $\mathrm{C}(22) \# 1-\mathrm{C}(16)-\mathrm{U}(1)$ & $125.8(4)$ & $\mathrm{C}(19)-\mathrm{C}(18)-\mathrm{C}(17)$ & 108.0 \\
\hline$C(17)-C(16)-U(1)$ & $74.3(3)$ & $\mathrm{C}(19) \# 1-\mathrm{C}(18)-\mathrm{C}(23)$ & $55.0(18)$ \\
\hline$C(15)-C(16)-U(1)$ & $76.7(3)$ & $\mathrm{C}(15) \# 1-\mathrm{C}(18)-\mathrm{C}(23)$ & $74.4(6)$ \\
\hline
\end{tabular}




\begin{tabular}{|c|c|c|c|}
\hline $\mathrm{C}(16) \# 1-\mathrm{C}(16)-\mathrm{U}(1)$ & $75.0(3)$ & $\mathrm{C}(15)-\mathrm{C}(19)-\mathrm{U}(1)$ & $75.4(2)$ \\
\hline $\mathrm{C}(18) \# 1-\mathrm{C}(18)-\mathrm{C}(23)$ & 144.4 & $\mathrm{C}(24)-\mathrm{C}(19)-\mathrm{U}(1)$ & $116.8(2)$ \\
\hline$C(19)-C(18)-C(23)$ & 126.0 & $C(22) \# 1-C(21)-C(16)$ & $53.5(7)$ \\
\hline$C(17)-C(18)-C(23)$ & 126.0 & $C(21) \# 1-C(22)-C(16) \# 1$ & $77.2(11)$ \\
\hline $\mathrm{C}(19) \# 1-\mathrm{C}(18)-\mathrm{C}(16) \# 1$ & $156.9(6)$ & $\mathrm{C}(21) \# 1-\mathrm{C}(22)-\mathrm{C}(17)$ & $91.9(9)$ \\
\hline$C(15) \# 1-C(18)-C(16) \# 1$ & $50.30(17)$ & $C(16) \# 1-C(22)-C(17)$ & $15.34(19)$ \\
\hline$C(18) \# 1-C(18)-C(16) \# 1$ & $92.1(5)$ & $\mathrm{C}(21) \# 1-\mathrm{C}(22)-\mathrm{C}(15) \# 1$ & $128.0(11)$ \\
\hline$C(19)-C(18)-C(16) \# 1$ & $110.4(5)$ & $C(16) \# 1-C(22)-C(15) \# 1$ & $54.5(2)$ \\
\hline$C(17)-C(18)-C(16) \# 1$ & $5.5(3)$ & $C(17)-C(22)-C(15) \# 1$ & $42.8(5)$ \\
\hline$C(23)-C(18)-C(16) \# 1$ & $123.4(5)$ & $\mathrm{C}(19) \# 1-\mathrm{C}(23)-\mathrm{C}(24) \# 1$ & $70.5(9)$ \\
\hline $\mathrm{C}(19) \# 1-\mathrm{C}(18)-\mathrm{U}(1)$ & $89.2(5)$ & $\mathrm{C}(19) \# 1-\mathrm{C}(23)-\mathrm{C}(18)$ & $17.83(16)$ \\
\hline $\mathrm{C}(15) \# 1-\mathrm{C}(18)-\mathrm{U}(1)$ & $80.0(5)$ & $\mathrm{C}(24) \# 1-\mathrm{C}(23)-\mathrm{C}(18)$ & $87.9(7)$ \\
\hline $\mathrm{C}(18) \# 1-\mathrm{C}(18)-\mathrm{U}(1)$ & $76.4(3)$ & $\mathrm{C}(19) \# 1-\mathrm{C}(23)-\mathrm{C}(15) \# 1$ & $56.9(3)$ \\
\hline $\mathrm{C}(19)-\mathrm{C}(18)-\mathrm{U}(1)$ & $76.7(3)$ & $\mathrm{C}(24) \# 1-\mathrm{C}(23)-\mathrm{C}(15) \# 1$ & $124.3(11)$ \\
\hline $\mathrm{C}(17)-\mathrm{C}(18)-\mathrm{U}(1)$ & $74.3(2)$ & $\mathrm{C}(18)-\mathrm{C}(23)-\mathrm{C}(15) \# 1$ & $42.2(5)$ \\
\hline $\mathrm{C}(23)-\mathrm{C}(18)-\mathrm{U}(1)$ & $115.3(2)$ & $C(23) \# 1-C(24)-C(19)$ & $53.9(6)$ \\
\hline $\mathrm{C}(16) \# 1-\mathrm{C}(18)-\mathrm{U}(1)$ & $70.6(3)$ & $\mathrm{C}(18)-\mathrm{C}(19)-\mathrm{U}(1)$ & $73.9(3)$ \\
\hline $\mathrm{C}(18) \# 1-\mathrm{C}(19)-\mathrm{C}(23) \# 1$ & 107.2(19) & & \\
\hline $\mathrm{C}(18) \# 1-\mathrm{C}(19)-\mathrm{C}(19) \# 1$ & $86.2(18)$ & & \\
\hline $\mathrm{C}(23) \# 1-\mathrm{C}(19)-\mathrm{C}(19) \# 1$ & $157.5(3)$ & & \\
\hline $\mathrm{C}(18) \# 1-\mathrm{C}(19)-\mathrm{C}(18)$ & $66.3(18)$ & & \\
\hline $\mathrm{C}(23) \# 1-\mathrm{C}(19)-\mathrm{C}(18)$ & $161.56(16)$ & & \\
\hline $\mathrm{C}(19) \# 1-\mathrm{C}(19)-\mathrm{C}(18)$ & 19.9 & & \\
\hline $\mathrm{C}(18) \# 1-\mathrm{C}(19)-\mathrm{C}(15)$ & $45.5(17)$ & & \\
\hline$C(23) \# 1-C(19)-C(15)$ & $73.4(3)$ & & \\
\hline $\mathrm{C}(19) \# 1-\mathrm{C}(19)-\mathrm{C}(15)$ & 126.7 & & \\
\hline $\mathrm{C}(18)-\mathrm{C}(19)-\mathrm{C}(15)$ & 108.0 & & \\
\hline $\mathrm{C}(18) \# 1-\mathrm{C}(19)-\mathrm{C}(24)$ & $159.7(12)$ & & \\
\hline $\mathrm{C}(23) \# 1-\mathrm{C}(19)-\mathrm{C}(24)$ & $55.7(2)$ & & \\
\hline $\mathrm{C}(19) \# 1-\mathrm{C}(19)-\mathrm{C}(24)$ & 107.0 & & \\
\hline$C(18)-C(19)-C(24)$ & 126.0 & & \\
\hline$C(15)-C(19)-C(24)$ & 126.0 & & \\
\hline $\mathrm{C}(18) \# 1-\mathrm{C}(19)-\mathrm{U}(1)$ & $80.9(5)$ & & \\
\hline $\mathrm{C}(23) \# 1-\mathrm{C}(19)-\mathrm{U}(1)$ & $123.2(4)$ & & \\
\hline $\mathrm{C}(19) \# 1-\mathrm{C}(19)-\mathrm{U}(1)$ & $75.9(3)$ & & \\
\hline
\end{tabular}


Figure S4: Thermal ellipsoid plot of one of the two symmetry-independent molecules in the asymmetric unit of $\left(\mathrm{C}_{5} \mathrm{Me}_{5}\right)_{2} \mathrm{U}\left[-\mathrm{N}=\mathrm{C}\left(\mathrm{CH}_{3}\right)\left(2,6-\mathrm{F}_{2}-\mathrm{C}_{6} \mathrm{H}_{3}\right)\right]_{2}(7)$ with ellipsoids projected at the $50 \%$ probability level.

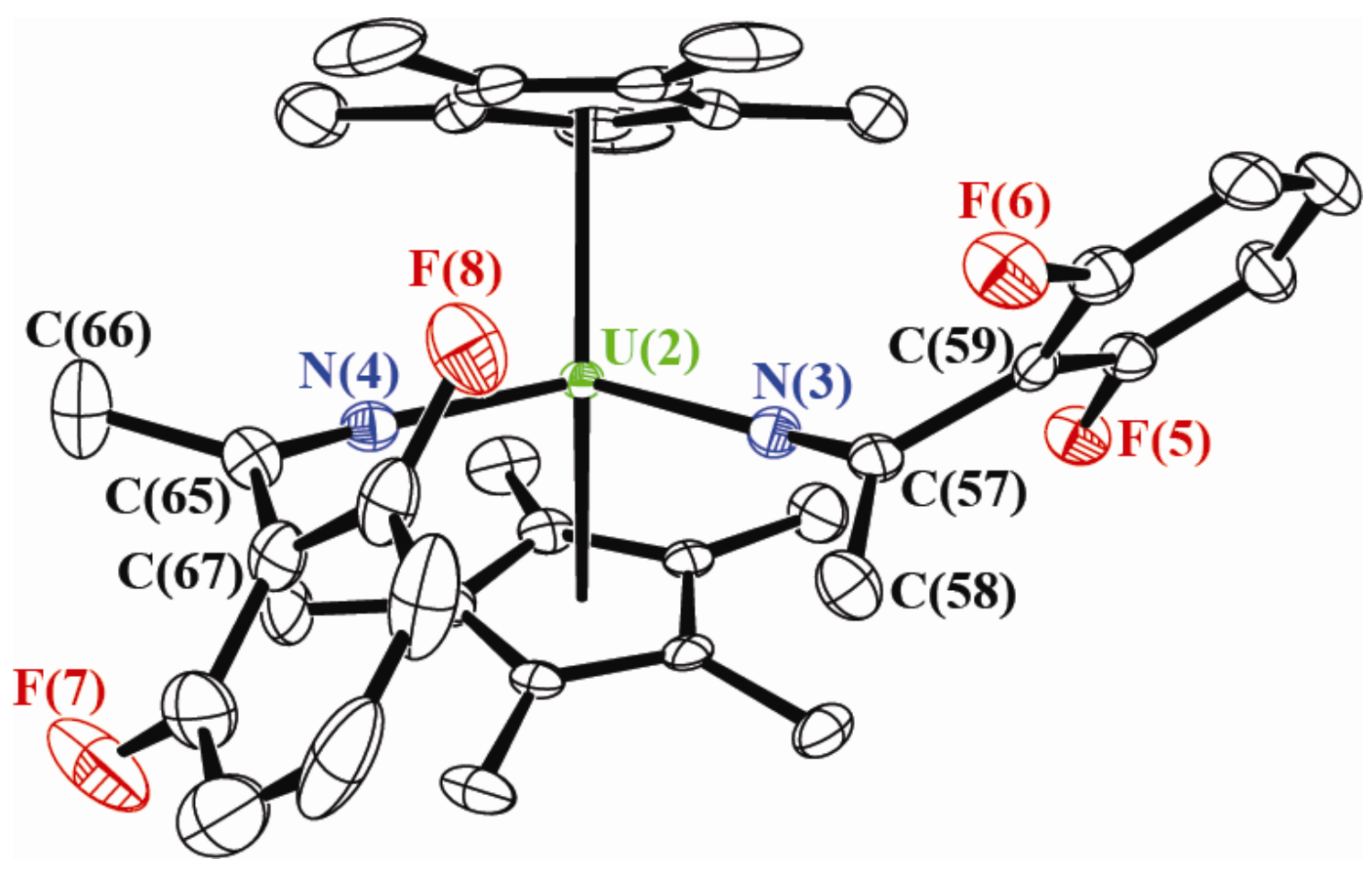


Table S7: Crystal Data and Structure Refinement for $\left(\mathrm{C}_{5} \mathrm{Me}_{5}\right)_{2} \mathrm{U}\left[-\mathrm{N}=\mathrm{C}\left(\mathrm{CH}_{3}\right)\left(2,6-\mathrm{F}_{2}-\mathrm{C}_{6} \mathrm{H}_{4}\right)\right]_{2}(7)$.

\begin{tabular}{|c|c|c|}
\hline Empirical formula & \multicolumn{2}{|l|}{$\mathrm{C}_{36} \mathrm{H}_{42} \mathrm{~F}_{4} \mathrm{~N}_{2} \mathrm{U}$} \\
\hline Formula weight & \multicolumn{2}{|l|}{816.75} \\
\hline Temperature & \multicolumn{2}{|l|}{$141(2) \mathrm{K}$} \\
\hline Wavelength & \multicolumn{2}{|l|}{$0.71073 \AA$} \\
\hline Crystal system & \multicolumn{2}{|l|}{ triclinic } \\
\hline Space group & \multicolumn{2}{|l|}{$\mathrm{P} \overline{1}$} \\
\hline \multirow[t]{3}{*}{ Unit cell dimensions } & $a=10.6877(6) \AA$ & $\alpha=108.793(1)^{\circ}$ \\
\hline & $\mathrm{b}=17.5713(9) \AA$ & $\beta=90.476(1)^{\circ}$ \\
\hline & $\mathrm{c}=19.3511(10) \AA$ & $\gamma=102.700(1)^{\circ}$ \\
\hline Volume & \multicolumn{2}{|l|}{$3343.8(3) \AA^{3}$} \\
\hline $\mathrm{Z}$ & \multicolumn{2}{|l|}{4} \\
\hline Density (calculated) & \multicolumn{2}{|l|}{$1.622 \mathrm{Mg} / \mathrm{m}^{3}$} \\
\hline Absorption coefficient & \multicolumn{2}{|l|}{$4.903 \mathrm{~mm}^{-1}$} \\
\hline $\mathrm{F}(000)$ & \multicolumn{2}{|l|}{1600} \\
\hline Crystal size & \multicolumn{2}{|c|}{$0.28 \times 0.12 \times 0.08 \mathrm{~mm}^{3}$} \\
\hline$\Theta$ Range for data collection & \multicolumn{2}{|l|}{1.12 to $28.95^{\circ}$} \\
\hline Index ranges & \multicolumn{2}{|c|}{$-14 \leq \mathrm{h} \leq 13,-23 \leq \mathrm{k} \leq 23,-26 \leq 1 \leq 26$} \\
\hline Reflections collected & \multicolumn{2}{|l|}{32791} \\
\hline Independent reflections & \multicolumn{2}{|c|}{$15667[\mathrm{R}(\mathrm{int})=0.0240]$} \\
\hline Completeness to $\Theta=25.00^{\circ}$ & \multicolumn{2}{|l|}{$99.3 \%$} \\
\hline Max. and min. transmission & \multicolumn{2}{|l|}{0.6951 and 0.3406} \\
\hline Refinement method & \multicolumn{2}{|c|}{ Full-matrix least-squares on $\mathrm{F}^{2}$} \\
\hline Data / restraints / parameters & \multicolumn{2}{|l|}{$15667 / 0 / 775$} \\
\hline Goodness-of-fit on $\mathrm{F}^{2}$ & \multicolumn{2}{|l|}{1.259} \\
\hline Final $R$ indices $[\mathrm{I}>2 \sigma(\mathrm{I})]$ & \multicolumn{2}{|c|}{$\mathrm{R} 1=0.0286, \mathrm{wR} 2=0.0689$} \\
\hline $\mathrm{R}$ indices (all data) & \multicolumn{2}{|c|}{$\mathrm{R} 1=0.0395, \mathrm{wR} 2=0.0757$} \\
\hline Largest diff. peak and hole & \multicolumn{2}{|c|}{1.724 and -1.041 e. $. \AA-3$} \\
\hline
\end{tabular}


Table S8: Bond Distances $[\AA]$ and Angles $\left[^{\circ}\right]$ for $\left(\mathrm{C}_{5} \mathrm{Me}_{5}\right)_{2} \mathrm{U}\left[-\mathrm{N}=\mathrm{C}\left(\mathrm{CH}_{3}\right)\left(2,6-\mathrm{F}_{2}-\mathrm{C}_{6} \mathrm{H}_{4}\right)\right]_{2}(7)$.

\begin{tabular}{|c|c|c|c|}
\hline $\mathrm{U}(1)-\mathrm{N}(1)$ & $2.185(3)$ & $\mathrm{N}(3)-\mathrm{C}(57)$ & $1.258(5)$ \\
\hline $\mathrm{U}(1)-\mathrm{N}(2)$ & $2.220(3)$ & $\mathrm{N}(4)-\mathrm{C}(65)$ & $1.265(5)$ \\
\hline $\mathrm{U}(1)-\mathrm{C}(1)$ & $2.704(4)$ & $C(1)-C(5)$ & $1.397(6)$ \\
\hline $\mathrm{U}(1)-\mathrm{C}(11)$ & $2.720(4)$ & $C(1)-C(2)$ & $1.407(7)$ \\
\hline $\mathrm{U}(1)-\mathrm{C}(2)$ & $2.720(4)$ & $C(1)-C(6)$ & $1.527(7)$ \\
\hline $\mathrm{U}(1)-\mathrm{C}(5)$ & $2.721(4)$ & $C(2)-C(3)$ & $1.407(8)$ \\
\hline $\mathrm{U}(1)-\mathrm{C}(12)$ & $2.733(4)$ & $C(2)-C(7)$ & $1.532(7)$ \\
\hline $\mathrm{U}(1)-\mathrm{C}(3)$ & $2.727(4)$ & $C(3)-C(4)$ & $1.408(8)$ \\
\hline $\mathrm{U}(1)-\mathrm{C}(4)$ & $2.737(4)$ & $C(3)-C(8)$ & $1.524(7)$ \\
\hline $\mathrm{U}(1)-\mathrm{C}(15)$ & $2.741(4)$ & $C(4)-C(5)$ & $1.381(6)$ \\
\hline $\mathrm{U}(1)-\mathrm{C}(13)$ & $2.747(4)$ & $C(4)-C(9)$ & $1.523(7)$ \\
\hline $\mathrm{U}(1)-\mathrm{C}(14)$ & $2.755(5)$ & $C(5)-C(10)$ & $1.503(7)$ \\
\hline $\mathrm{U}(2)-\mathrm{N}(4)$ & $2.185(3)$ & $\mathrm{C}(11)-\mathrm{C}(15)$ & $1.371(8)$ \\
\hline $\mathrm{U}(2)-\mathrm{N}(3)$ & $2.190(3)$ & $\mathrm{C}(11)-\mathrm{C}(12)$ & $1.461(9)$ \\
\hline $\mathrm{U}(2)-\mathrm{C}(39)$ & $2.732(4)$ & $\mathrm{C}(11)-\mathrm{C}(16)$ & $1.495(8)$ \\
\hline $\mathrm{U}(2)-\mathrm{C}(38)$ & $2.740(3)$ & $\mathrm{C}(12)-\mathrm{C}(13)$ & $1.440(9)$ \\
\hline $\mathrm{U}(2)-\mathrm{C}(49)$ & $2.738(4)$ & $\mathrm{C}(12)-\mathrm{C}(17)$ & $1.491(8)$ \\
\hline $\mathrm{U}(2)-\mathrm{C}(48)$ & $2.737(4)$ & $\mathrm{C}(13)-\mathrm{C}(14)$ & $1.323(8)$ \\
\hline $\mathrm{U}(2)-\mathrm{C}(37)$ & $2.743(4)$ & $\mathrm{C}(13)-\mathrm{C}(18)$ & $1.514(8)$ \\
\hline $\mathrm{U}(2)-\mathrm{C}(47)$ & $2.743(4)$ & $\mathrm{C}(14)-\mathrm{C}(15)$ & $1.305(7)$ \\
\hline $\mathrm{U}(2)-\mathrm{C}(50)$ & $2.750(4)$ & C(14)-C(19) & $1.526(7)$ \\
\hline $\mathrm{U}(2)-\mathrm{C}(40)$ & $2.752(4)$ & $C(15)-C(20)$ & $1.547(8)$ \\
\hline $\mathrm{U}(2)-\mathrm{C}(41)$ & $2.752(4)$ & $\mathrm{C}(21)-\mathrm{C}(22)$ & $1.502(6)$ \\
\hline $\mathrm{U}(2)-\mathrm{C}(51)$ & $2.759(4)$ & $\mathrm{C}(21)-\mathrm{C}(23)$ & $1.519(6)$ \\
\hline $\mathrm{F}(1)-\mathrm{C}(24)$ & $1.365(6)$ & $\mathrm{C}(23)-\mathrm{C}(28)$ & $1.381(6)$ \\
\hline $\mathrm{F}(2)-\mathrm{C}(28)$ & $1.343(5)$ & $\mathrm{C}(23)-\mathrm{C}(24)$ & $1.390(6)$ \\
\hline $\mathrm{F}(3)-\mathrm{C}(32)$ & $1.347(5)$ & $\mathrm{C}(24)-\mathrm{C}(25)$ & $1.380(7)$ \\
\hline F(4)-C(36) & $1.367(6)$ & $C(25)-C(26)$ & $1.345(8)$ \\
\hline $\mathrm{F}(5)-\mathrm{C}(60)$ & $1.363(4)$ & $\mathrm{C}(26)-\mathrm{C}(27)$ & $1.383(8)$ \\
\hline $\mathrm{F}(6)-\mathrm{C}(64)$ & $1.370(4)$ & $\mathrm{C}(27)-\mathrm{C}(28)$ & $1.406(7)$ \\
\hline $\mathrm{F}(7)-\mathrm{C}(68)$ & $1.373(6)$ & C(29)-C(30) & $1.519(6)$ \\
\hline $\mathrm{F}(8)-\mathrm{C}(72)$ & $1.353(5)$ & C(29)-C(31) & $1.524(5)$ \\
\hline $\mathrm{N}(1)-\mathrm{C}(21)$ & $1.271(5)$ & $\mathrm{C}(31)-\mathrm{C}(32)$ & $1.369(6)$ \\
\hline $\mathrm{N}(2)-\mathrm{C}(29)$ & $1.251(5)$ & $\mathrm{C}(31)-\mathrm{C}(36)$ & $1.377(6)$ \\
\hline
\end{tabular}




\begin{tabular}{|c|c|c|c|}
\hline C(32)-C(33) & $1.385(6)$ & $C(67)-C(68)$ & $1.383(6)$ \\
\hline C(33)-C(34) & $1.360(7)$ & $\mathrm{C}(68)-\mathrm{C}(69)$ & $1.372(7)$ \\
\hline C(34)-C(35) & $1.372(7)$ & $\mathrm{C}(69)-\mathrm{C}(70)$ & $1.385(9)$ \\
\hline C(35)-C(36) & $1.362(7)$ & $\mathrm{C}(70)-\mathrm{C}(71)$ & $1.353(8)$ \\
\hline C(37)-C(38) & $1.407(5)$ & $\mathrm{C}(71)-\mathrm{C}(72)$ & $1.379(6)$ \\
\hline C(37)-C(41) & $1.410(5)$ & & \\
\hline C(37)-C(42) & $1.511(5)$ & $\mathrm{N}(1)-\mathrm{U}(1)-\mathrm{N}(2)$ & $107.51(12)$ \\
\hline C(38)-C(39) & $1.416(5)$ & $\mathrm{N}(1)-\mathrm{U}(1)-\mathrm{C}(1)$ & $122.61(14)$ \\
\hline C(38)-C(43) & $1.507(5)$ & $\mathrm{N}(2)-\mathrm{U}(1)-\mathrm{C}(1)$ & $100.94(14)$ \\
\hline C(39)-C(40) & $1.412(5)$ & $\mathrm{N}(1)-\mathrm{U}(1)-\mathrm{C}(11)$ & $90.93(18)$ \\
\hline C(39)-C(44) & $1.502(5)$ & $\mathrm{N}(2)-\mathrm{U}(1)-\mathrm{C}(11)$ & $127.11(13)$ \\
\hline $\mathrm{C}(40)-\mathrm{C}(41)$ & $1.419(5)$ & $\mathrm{C}(1)-\mathrm{U}(1)-\mathrm{C}(11)$ & 109.81(17) \\
\hline C(40)-C(45) & $1.501(5)$ & $\mathrm{N}(1)-\mathrm{U}(1)-\mathrm{C}(2)$ & $97.00(17)$ \\
\hline $\mathrm{C}(41)-\mathrm{C}(46)$ & $1.521(6)$ & $\mathrm{N}(2)-\mathrm{U}(1)-\mathrm{C}(2)$ & $127.45(13)$ \\
\hline $\mathrm{C}(47)-\mathrm{C}(51)$ & $1.415(6)$ & $C(1)-U(1)-C(2)$ & $30.07(15)$ \\
\hline $\mathrm{C}(47)-\mathrm{C}(48)$ & $1.416(6)$ & $\mathrm{C}(11)-\mathrm{U}(1)-\mathrm{C}(2)$ & $97.26(16)$ \\
\hline $\mathrm{C}(47)-\mathrm{C}(52)$ & $1.513(6)$ & $\mathrm{N}(1)-\mathrm{U}(1)-\mathrm{C}(5)$ & $111.85(13)$ \\
\hline C(48)-C(49) & $1.407(6)$ & $\mathrm{N}(2)-\mathrm{U}(1)-\mathrm{C}(5)$ & $78.66(12)$ \\
\hline $\mathrm{C}(48)-\mathrm{C}(53)$ & $1.508(6)$ & $\mathrm{C}(1)-\mathrm{U}(1)-\mathrm{C}(5)$ & $29.85(12)$ \\
\hline C(49)-C(50) & $1.405(6)$ & $\mathrm{C}(11)-\mathrm{U}(1)-\mathrm{C}(5)$ & $139.65(17)$ \\
\hline C(49)-C(54) & $1.515(6)$ & $C(2)-U(1)-C(5)$ & $48.95(13)$ \\
\hline $\mathrm{C}(50)-\mathrm{C}(51)$ & $1.409(6)$ & $\mathrm{N}(1)-\mathrm{U}(1)-\mathrm{C}(12)$ & $122.0(2)$ \\
\hline C(50)-C(55) & $1.499(6)$ & $\mathrm{N}(2)-\mathrm{U}(1)-\mathrm{C}(12)$ & $109.7(2)$ \\
\hline $\mathrm{C}(51)-\mathrm{C}(56)$ & $1.513(6)$ & $\mathrm{C}(1)-\mathrm{U}(1)-\mathrm{C}(12)$ & $91.70(13)$ \\
\hline C(57)-C(59) & $1.515(5)$ & $C(11)-U(1)-C(12)$ & $31.1(2)$ \\
\hline $\mathrm{C}(57)-\mathrm{C}(58)$ & $1.516(5)$ & $\mathrm{C}(2)-\mathrm{U}(1)-\mathrm{C}(12)$ & $94.02(18)$ \\
\hline C(59)-C(64) & $1.387(5)$ & $\mathrm{C}(5)-\mathrm{U}(1)-\mathrm{C}(12)$ & $117.91(15)$ \\
\hline $\mathrm{C}(59)-\mathrm{C}(60)$ & $1.390(5)$ & $\mathrm{N}(1)-\mathrm{U}(1)-\mathrm{C}(3)$ & $73.12(14)$ \\
\hline$C(60)-C(61)$ & $1.372(6)$ & $\mathrm{N}(2)-\mathrm{U}(1)-\mathrm{C}(3)$ & $117.95(17)$ \\
\hline $\mathrm{C}(61)-\mathrm{C}(62)$ & $1.380(6)$ & $C(1)-U(1)-C(3)$ & $49.51(15)$ \\
\hline$C(62)-C(63)$ & $1.382(6)$ & $\mathrm{C}(11)-\mathrm{U}(1)-\mathrm{C}(3)$ & $114.78(19)$ \\
\hline $\mathrm{C}(63)-\mathrm{C}(64)$ & $1.360(6)$ & $C(2)-U(1)-C(3)$ & $29.93(18)$ \\
\hline$C(65)-C(67)$ & $1.510(5)$ & $C(5)-U(1)-C(3)$ & $48.72(14)$ \\
\hline $\mathrm{C}(65)-\mathrm{C}(66)$ & $1.514(6)$ & $\mathrm{C}(12)-\mathrm{U}(1)-\mathrm{C}(3)$ & $121.9(2)$ \\
\hline \multirow[t]{2}{*}{$\mathrm{C}(67)-\mathrm{C}(72)$} & $1.375(6)$ & $\mathrm{N}(1)-\mathrm{U}(1)-\mathrm{C}(4)$ & $82.55(14)$ \\
\hline & & $\mathrm{N}(2)-\mathrm{U}(1)-\mathrm{C}(4)$ & $88.14(14)$ \\
\hline
\end{tabular}




\begin{tabular}{|c|c|c|c|}
\hline $\mathrm{C}(1)-\mathrm{U}(1)-\mathrm{C}(4)$ & $49.38(13)$ & $\mathrm{C}(13)-\mathrm{U}(1)-\mathrm{C}(14)$ & $27.83(16)$ \\
\hline $\mathrm{C}(11)-\mathrm{U}(1)-\mathrm{C}(4)$ & $144.22(15)$ & $\mathrm{N}(4)-\mathrm{U}(2)-\mathrm{N}(3)$ & $104.02(12)$ \\
\hline $\mathrm{C}(2)-\mathrm{U}(1)-\mathrm{C}(4)$ & $49.32(15)$ & N(4)-U(2)-C(39) & $124.22(12)$ \\
\hline$C(5)-U(1)-C(4)$ & $29.32(14)$ & $\mathrm{N}(3)-\mathrm{U}(2)-\mathrm{C}(39)$ & $92.91(12)$ \\
\hline $\mathrm{C}(12)-\mathrm{U}(1)-\mathrm{C}(4)$ & $140.35(14)$ & $\mathrm{N}(4)-\mathrm{U}(2)-\mathrm{C}(38)$ & $105.59(12)$ \\
\hline $\mathrm{C}(3)-\mathrm{U}(1)-\mathrm{C}(4)$ & $29.86(17)$ & $\mathrm{N}(3)-\mathrm{U}(2)-\mathrm{C}(38)$ & $74.33(11)$ \\
\hline N(1)-U(1)-C(15) & $79.21(14)$ & $\mathrm{C}(39)-\mathrm{U}(2)-\mathrm{C}(38)$ & $29.99(11)$ \\
\hline N(2)-U(1)-C(15) & $105.38(15)$ & $\mathrm{N}(4)-\mathrm{U}(2)-\mathrm{C}(49)$ & $78.94(12)$ \\
\hline C(1)-U(1)-C(15) & $138.17(14)$ & $\mathrm{N}(3)-\mathrm{U}(2)-\mathrm{C}(49)$ & $104.84(13)$ \\
\hline $\mathrm{C}(11)-\mathrm{U}(1)-\mathrm{C}(15)$ & $29.07(17)$ & $\mathrm{C}(39)-\mathrm{U}(2)-\mathrm{C}(49)$ & $146.73(12)$ \\
\hline C(2)-U(1)-C(15) & $124.79(15)$ & $\mathrm{C}(38)-\mathrm{U}(2)-\mathrm{C}(49)$ & $175.47(12)$ \\
\hline $\mathrm{C}(5)-\mathrm{U}(1)-\mathrm{C}(15)$ & $166.87(14)$ & N(4)-U(2)-C(48) & $101.61(13)$ \\
\hline $\mathrm{C}(12)-\mathrm{U}(1)-\mathrm{C}(15)$ & $48.98(15)$ & $\mathrm{N}(3)-\mathrm{U}(2)-\mathrm{C}(48)$ & $80.82(12)$ \\
\hline $\mathrm{C}(3)-\mathrm{U}(1)-\mathrm{C}(15)$ & $133.68(18)$ & $\mathrm{C}(39)-\mathrm{U}(2)-\mathrm{C}(48)$ & $133.71(13)$ \\
\hline $\mathrm{C}(4)-\mathrm{U}(1)-\mathrm{C}(15)$ & $159.88(17)$ & $\mathrm{C}(38)-\mathrm{U}(2)-\mathrm{C}(48)$ & $146.72(13)$ \\
\hline N(1)-U(1)-C(13) & $124.46(14)$ & $\mathrm{C}(49)-\mathrm{U}(2)-\mathrm{C}(48)$ & $29.77(13)$ \\
\hline N(2)-U(1)-C(13) & $81.28(14)$ & $\mathrm{N}(4)-\mathrm{U}(2)-\mathrm{C}(37)$ & $77.53(12)$ \\
\hline $\mathrm{C}(1)-\mathrm{U}(1)-\mathrm{C}(13)$ & $108.18(17)$ & $\mathrm{N}(3)-\mathrm{U}(2)-\mathrm{C}(37)$ & $90.39(12)$ \\
\hline $\mathrm{C}(11)-\mathrm{U}(1)-\mathrm{C}(13)$ & $48.97(16)$ & $\mathrm{C}(39)-\mathrm{U}(2)-\mathrm{C}(37)$ & $49.18(11)$ \\
\hline $\mathrm{C}(2)-\mathrm{U}(1)-\mathrm{C}(13)$ & 121.1(2) & $\mathrm{C}(38)-\mathrm{U}(2)-\mathrm{C}(37)$ & $29.73(11)$ \\
\hline C(5)-U(1)-C(13) & $123.56(15)$ & $\mathrm{C}(49)-\mathrm{U}(2)-\mathrm{C}(37)$ & $154.47(12)$ \\
\hline $\mathrm{C}(12)-\mathrm{U}(1)-\mathrm{C}(13)$ & $30.47(19)$ & $\mathrm{C}(48)-\mathrm{U}(2)-\mathrm{C}(37)$ & $170.73(12)$ \\
\hline $\mathrm{C}(3)-\mathrm{U}(1)-\mathrm{C}(13)$ & $150.8(2)$ & $\mathrm{N}(4)-\mathrm{U}(2)-\mathrm{C}(47)$ & $127.91(12)$ \\
\hline $\mathrm{C}(4)-\mathrm{U}(1)-\mathrm{C}(13)$ & $152.85(16)$ & $\mathrm{N}(3)-\mathrm{U}(2)-\mathrm{C}(47)$ & $88.60(13)$ \\
\hline $\mathrm{C}(15)-\mathrm{U}(1)-\mathrm{C}(13)$ & $46.55(16)$ & $\mathrm{C}(39)-\mathrm{U}(2)-\mathrm{C}(47)$ & $104.82(12)$ \\
\hline N(1)-U(1)-C(14) & $97.83(16)$ & $\mathrm{C}(38)-\mathrm{U}(2)-\mathrm{C}(47)$ & $126.41(12)$ \\
\hline N(2)-U(1)-C(14) & $80.63(14)$ & $\mathrm{C}(49)-\mathrm{U}(2)-\mathrm{C}(47)$ & $49.07(12)$ \\
\hline C(1)-U(1)-C(14) & $135.86(16)$ & $\mathrm{C}(48)-\mathrm{U}(2)-\mathrm{C}(47)$ & $29.96(13)$ \\
\hline $\mathrm{C}(11)-\mathrm{U}(1)-\mathrm{C}(14)$ & $47.38(15)$ & $\mathrm{C}(37)-\mathrm{U}(2)-\mathrm{C}(47)$ & $153.89(12)$ \\
\hline $\mathrm{C}(2)-\mathrm{U}(1)-\mathrm{C}(14)$ & $141.59(16)$ & $\mathrm{N}(4)-\mathrm{U}(2)-\mathrm{C}(50)$ & $88.49(12)$ \\
\hline C(5)-U(1)-C(14) & $147.74(16)$ & $\mathrm{N}(3)-\mathrm{U}(2)-\mathrm{C}(50)$ & $130.01(12)$ \\
\hline $\mathrm{C}(12)-\mathrm{U}(1)-\mathrm{C}(14)$ & $48.43(15)$ & $\mathrm{C}(39)-\mathrm{U}(2)-\mathrm{C}(50)$ & $119.66(12)$ \\
\hline $\mathrm{C}(3)-\mathrm{U}(1)-\mathrm{C}(14)$ & $160.86(18)$ & $\mathrm{C}(38)-\mathrm{U}(2)-\mathrm{C}(50)$ & $148.71(12)$ \\
\hline $\mathrm{C}(4)-\mathrm{U}(1)-\mathrm{C}(14)$ & $168.35(16)$ & $\mathrm{C}(49)-\mathrm{U}(2)-\mathrm{C}(50)$ & $29.66(13)$ \\
\hline $\mathrm{C}(15)-\mathrm{U}(1)-\mathrm{C}(14)$ & $27.47(16)$ & $\mathrm{C}(48)-\mathrm{U}(2)-\mathrm{C}(50)$ & $49.18(13)$ \\
\hline
\end{tabular}




\begin{tabular}{|c|c|c|c|}
\hline $\mathrm{C}(37)-\mathrm{U}(2)-\mathrm{C}(50)$ & $139.52(12)$ & $\mathrm{C}(65)-\mathrm{N}(4)-\mathrm{U}(2)$ & 171.7(3) \\
\hline $\mathrm{C}(47)-\mathrm{U}(2)-\mathrm{C}(50)$ & $49.07(12)$ & $\mathrm{C}(5)-\mathrm{C}(1)-\mathrm{C}(2)$ & $107.0(4)$ \\
\hline $\mathrm{N}(4)-\mathrm{U}(2)-\mathrm{C}(40)$ & 104.73(12) & $C(5)-C(1)-C(6)$ & $122.7(5)$ \\
\hline $\mathrm{N}(3)-\mathrm{U}(2)-\mathrm{C}(40)$ & $121.52(11)$ & $C(2)-C(1)-C(6)$ & $130.0(5)$ \\
\hline $\mathrm{C}(39)-\mathrm{U}(2)-\mathrm{C}(40)$ & 29.83(11) & $\mathrm{C}(5)-\mathrm{C}(1)-\mathrm{U}(1)$ & $75.8(2)$ \\
\hline $\mathrm{C}(38)-\mathrm{U}(2)-\mathrm{C}(40)$ & $49.30(11)$ & $\mathrm{C}(2)-\mathrm{C}(1)-\mathrm{U}(1)$ & $75.6(2)$ \\
\hline $\mathrm{C}(49)-\mathrm{U}(2)-\mathrm{C}(40)$ & 130.01(12) & $\mathrm{C}(6)-\mathrm{C}(1)-\mathrm{U}(1)$ & 119.7(3) \\
\hline $\mathrm{C}(48)-\mathrm{U}(2)-\mathrm{C}(40)$ & $138.91(12)$ & $\mathrm{C}(3)-\mathrm{C}(2)-\mathrm{C}(1)$ & $107.9(4)$ \\
\hline $\mathrm{C}(37)-\mathrm{U}(2)-\mathrm{C}(40)$ & $49.09(11)$ & $C(3)-C(2)-C(7)$ & $128.4(7)$ \\
\hline $\mathrm{C}(47)-\mathrm{U}(2)-\mathrm{C}(40)$ & $110.83(13)$ & $\mathrm{C}(1)-\mathrm{C}(2)-\mathrm{C}(7)$ & $123.5(7)$ \\
\hline $\mathrm{C}(50)-\mathrm{U}(2)-\mathrm{C}(40)$ & $100.50(12)$ & $\mathrm{C}(3)-\mathrm{C}(2)-\mathrm{U}(1)$ & 75.3(3) \\
\hline $\mathrm{N}(4)-\mathrm{U}(2)-\mathrm{C}(41)$ & $76.81(12)$ & $\mathrm{C}(1)-\mathrm{C}(2)-\mathrm{U}(1)$ & $74.3(2)$ \\
\hline $\mathrm{N}(3)-\mathrm{U}(2)-\mathrm{C}(41)$ & $119.68(12)$ & $\mathrm{C}(7)-\mathrm{C}(2)-\mathrm{U}(1)$ & $120.4(3)$ \\
\hline $\mathrm{C}(39)-\mathrm{U}(2)-\mathrm{C}(41)$ & $49.32(11)$ & $C(2)-C(3)-C(4)$ & $108.0(4)$ \\
\hline $\mathrm{C}(38)-\mathrm{U}(2)-\mathrm{C}(41)$ & $49.26(11)$ & $\mathrm{C}(2)-\mathrm{C}(3)-\mathrm{C}(8)$ & $125.5(7)$ \\
\hline $\mathrm{C}(49)-\mathrm{U}(2)-\mathrm{C}(41)$ & $133.09(13)$ & $\mathrm{C}(4)-\mathrm{C}(3)-\mathrm{C}(8)$ & $126.1(8)$ \\
\hline $\mathrm{C}(48)-\mathrm{U}(2)-\mathrm{C}(41)$ & $159.38(12)$ & $\mathrm{C}(2)-\mathrm{C}(3)-\mathrm{U}(1)$ & $74.7(3)$ \\
\hline $\mathrm{C}(37)-\mathrm{U}(2)-\mathrm{C}(41)$ & $29.73(11)$ & $\mathrm{C}(4)-\mathrm{C}(3)-\mathrm{U}(1)$ & $75.5(2)$ \\
\hline $\mathrm{C}(47)-\mathrm{U}(2)-\mathrm{C}(41)$ & $138.84(13)$ & $\mathrm{C}(8)-\mathrm{C}(3)-\mathrm{U}(1)$ & 121.7(3) \\
\hline $\mathrm{C}(50)-\mathrm{U}(2)-\mathrm{C}(41)$ & $110.27(12)$ & $C(5)-C(4)-C(3)$ & $107.4(4)$ \\
\hline $\mathrm{C}(40)-\mathrm{U}(2)-\mathrm{C}(41)$ & 29.88(11) & $\mathrm{C}(5)-\mathrm{C}(4)-\mathrm{C}(9)$ & $124.9(6)$ \\
\hline $\mathrm{N}(4)-\mathrm{U}(2)-\mathrm{C}(51)$ & $118.06(12)$ & $\mathrm{C}(3)-\mathrm{C}(4)-\mathrm{C}(9)$ & $127.7(6)$ \\
\hline $\mathrm{N}(3)-\mathrm{U}(2)-\mathrm{C}(51)$ & 118.12(13) & $\mathrm{C}(5)-\mathrm{C}(4)-\mathrm{U}(1)$ & $74.7(2)$ \\
\hline $\mathrm{C}(39)-\mathrm{U}(2)-\mathrm{C}(51)$ & $97.88(11)$ & $\mathrm{C}(3)-\mathrm{C}(4)-\mathrm{U}(1)$ & $74.7(3)$ \\
\hline $\mathrm{C}(38)-\mathrm{U}(2)-\mathrm{C}(51)$ & $127.32(12)$ & $\mathrm{C}(9)-\mathrm{C}(4)-\mathrm{U}(1)$ & 117.1(3) \\
\hline $\mathrm{C}(49)-\mathrm{U}(2)-\mathrm{C}(51)$ & $48.95(12)$ & $C(4)-C(5)-C(1)$ & $109.8(4)$ \\
\hline $\mathrm{C}(48)-\mathrm{U}(2)-\mathrm{C}(51)$ & $49.28(12)$ & $C(4)-C(5)-C(10)$ & $124.5(5)$ \\
\hline $\mathrm{C}(37)-\mathrm{U}(2)-\mathrm{C}(51)$ & $139.29(12)$ & $C(1)-C(5)-C(10)$ & $125.5(5)$ \\
\hline $\mathrm{C}(47)-\mathrm{U}(2)-\mathrm{C}(51)$ & $29.80(13)$ & $\mathrm{C}(4)-\mathrm{C}(5)-\mathrm{U}(1)$ & $76.0(2)$ \\
\hline $\mathrm{C}(50)-\mathrm{U}(2)-\mathrm{C}(51)$ & 29.64(12) & $\mathrm{C}(1)-\mathrm{C}(5)-\mathrm{U}(1)$ & $74.4(2)$ \\
\hline $\mathrm{C}(40)-\mathrm{U}(2)-\mathrm{C}(51)$ & $90.25(12)$ & $C(10)-C(5)-U(1)$ & 121.1(3) \\
\hline $\mathrm{C}(41)-\mathrm{U}(2)-\mathrm{C}(51)$ & $112.88(12)$ & $\mathrm{C}(15)-\mathrm{C}(11)-\mathrm{C}(12)$ & $106.5(5)$ \\
\hline $\mathrm{C}(21)-\mathrm{N}(1)-\mathrm{U}(1)$ & $172.8(3)$ & $C(15)-C(11)-C(16)$ & $126.9(8)$ \\
\hline $\mathrm{C}(29)-\mathrm{N}(2)-\mathrm{U}(1)$ & $176.8(3)$ & $\mathrm{C}(12)-\mathrm{C}(11)-\mathrm{C}(16)$ & $126.3(8)$ \\
\hline$C(57)-N(3)-U(2)$ & $169.5(3)$ & $\mathrm{C}(15)-\mathrm{C}(11)-\mathrm{U}(1)$ & $76.3(3)$ \\
\hline
\end{tabular}




\begin{tabular}{|c|c|c|c|}
\hline $\mathrm{C}(12)-\mathrm{C}(11)-\mathrm{U}(1)$ & $75.0(2)$ & & \\
\hline$C(16)-C(11)-U(1)$ & $119.4(4)$ & $C(26)-C(25)-C(24)$ & $119.0(5)$ \\
\hline $\mathrm{C}(13)-\mathrm{C}(12)-\mathrm{C}(11)$ & $102.7(4)$ & $C(25)-C(26)-C(27)$ & $121.2(5)$ \\
\hline$C(13)-C(12)-C(17)$ & $130.0(11)$ & $\mathrm{C}(26)-\mathrm{C}(27)-\mathrm{C}(28)$ & $118.0(5)$ \\
\hline $\mathrm{C}(11)-\mathrm{C}(12)-\mathrm{C}(17)$ & $126.0(10)$ & $F(2)-C(28)-C(23)$ & $118.2(4)$ \\
\hline $\mathrm{C}(13)-\mathrm{C}(12)-\mathrm{U}(1)$ & $75.3(2)$ & $\mathrm{F}(2)-\mathrm{C}(28)-\mathrm{C}(27)$ & $118.8(5)$ \\
\hline $\mathrm{C}(11)-\mathrm{C}(12)-\mathrm{U}(1)$ & $74.0(3)$ & $\mathrm{C}(23)-\mathrm{C}(28)-\mathrm{C}(27)$ & $123.1(5)$ \\
\hline $\mathrm{C}(17)-\mathrm{C}(12)-\mathrm{U}(1)$ & $124.5(4)$ & $\mathrm{N}(2)-\mathrm{C}(29)-\mathrm{C}(30)$ & $125.7(4)$ \\
\hline$C(14)-C(13)-C(12)$ & $109.0(5)$ & $\mathrm{N}(2)-\mathrm{C}(29)-\mathrm{C}(31)$ & $120.9(4)$ \\
\hline$C(14)-C(13)-C(18)$ & $127.4(8)$ & $C(30)-C(29)-C(31)$ & $113.4(3)$ \\
\hline$C(12)-C(13)-C(18)$ & $123.4(8)$ & $C(32)-C(31)-C(36)$ & $113.2(4)$ \\
\hline $\mathrm{C}(14)-\mathrm{C}(13)-\mathrm{U}(1)$ & $76.4(3)$ & $\mathrm{C}(32)-\mathrm{C}(31)-\mathrm{C}(29)$ & $124.5(4)$ \\
\hline $\mathrm{C}(12)-\mathrm{C}(13)-\mathrm{U}(1)$ & $74.2(3)$ & $C(36)-C(31)-C(29)$ & $122.3(4)$ \\
\hline $\mathrm{C}(18)-\mathrm{C}(13)-\mathrm{U}(1)$ & $118.8(3)$ & $\mathrm{F}(3)-\mathrm{C}(32)-\mathrm{C}(31)$ & $117.5(4)$ \\
\hline$C(15)-C(14)-C(13)$ & $111.2(5)$ & $\mathrm{F}(3)-\mathrm{C}(32)-\mathrm{C}(33)$ & $117.8(4)$ \\
\hline$C(15)-C(14)-C(19)$ & $124.1(6)$ & $\mathrm{C}(31)-\mathrm{C}(32)-\mathrm{C}(33)$ & $124.7(4)$ \\
\hline$C(13)-C(14)-C(19)$ & $124.5(6)$ & $\mathrm{C}(34)-\mathrm{C}(33)-\mathrm{C}(32)$ & $118.1(4)$ \\
\hline $\mathrm{C}(15)-\mathrm{C}(14)-\mathrm{U}(1)$ & $75.7(3)$ & $\mathrm{C}(33)-\mathrm{C}(34)-\mathrm{C}(35)$ & $120.4(4)$ \\
\hline $\mathrm{C}(13)-\mathrm{C}(14)-\mathrm{U}(1)$ & $75.8(3)$ & $\mathrm{C}(36)-\mathrm{C}(35)-\mathrm{C}(34)$ & $118.2(5)$ \\
\hline $\mathrm{C}(19)-\mathrm{C}(14)-\mathrm{U}(1)$ & $118.9(3)$ & $\mathrm{F}(4)-\mathrm{C}(36)-\mathrm{C}(35)$ & $119.0(4)$ \\
\hline$C(14)-C(15)-C(11)$ & $110.6(5)$ & $\mathrm{F}(4)-\mathrm{C}(36)-\mathrm{C}(31)$ & $115.7(4)$ \\
\hline$C(14)-C(15)-C(20)$ & $127.7(6)$ & $\mathrm{C}(35)-\mathrm{C}(36)-\mathrm{C}(31)$ & $125.3(5)$ \\
\hline $\mathrm{C}(11)-\mathrm{C}(15)-\mathrm{C}(20)$ & $121.6(6)$ & $\mathrm{C}(38)-\mathrm{C}(37)-\mathrm{C}(41)$ & $108.7(3)$ \\
\hline $\mathrm{C}(14)-\mathrm{C}(15)-\mathrm{U}(1)$ & $76.8(3)$ & $\mathrm{C}(38)-\mathrm{C}(37)-\mathrm{C}(42)$ & $125.3(4)$ \\
\hline $\mathrm{C}(11)-\mathrm{C}(15)-\mathrm{U}(1)$ & $74.6(3)$ & $\mathrm{C}(41)-\mathrm{C}(37)-\mathrm{C}(42)$ & $125.8(4)$ \\
\hline $\mathrm{C}(20)-\mathrm{C}(15)-\mathrm{U}(1)$ & 119.1(3) & $\mathrm{C}(38)-\mathrm{C}(37)-\mathrm{U}(2)$ & $75.0(2)$ \\
\hline $\mathrm{N}(1)-\mathrm{C}(21)-\mathrm{C}(22)$ & $124.3(4)$ & $\mathrm{C}(41)-\mathrm{C}(37)-\mathrm{U}(2)$ & $75.5(2)$ \\
\hline $\mathrm{N}(1)-\mathrm{C}(21)-\mathrm{C}(23)$ & 119.2(4) & $\mathrm{C}(42)-\mathrm{C}(37)-\mathrm{U}(2)$ & $119.5(3)$ \\
\hline $\mathrm{C}(22)-\mathrm{C}(21)-\mathrm{C}(23)$ & $116.4(4)$ & $\mathrm{C}(37)-\mathrm{C}(38)-\mathrm{C}(39)$ & $107.6(3)$ \\
\hline $\mathrm{C}(28)-\mathrm{C}(23)-\mathrm{C}(24)$ & $114.8(4)$ & $\mathrm{C}(37)-\mathrm{C}(38)-\mathrm{C}(43)$ & $125.8(4)$ \\
\hline $\mathrm{C}(28)-\mathrm{C}(23)-\mathrm{C}(21)$ & $121.9(4)$ & $\mathrm{C}(39)-\mathrm{C}(38)-\mathrm{C}(43)$ & $126.1(4)$ \\
\hline$C(24)-C(23)-C(21)$ & $123.3(4)$ & $\mathrm{C}(37)-\mathrm{C}(38)-\mathrm{U}(2)$ & $75.2(2)$ \\
\hline $\mathrm{F}(1)-\mathrm{C}(24)-\mathrm{C}(25)$ & $118.7(5)$ & $\mathrm{C}(39)-\mathrm{C}(38)-\mathrm{U}(2)$ & $74.7(2)$ \\
\hline $\mathrm{F}(1)-\mathrm{C}(24)-\mathrm{C}(23)$ & $117.3(4)$ & $\mathrm{C}(43)-\mathrm{C}(38)-\mathrm{U}(2)$ & $122.5(3)$ \\
\hline$C(25)-C(24)-C(23)$ & $124.0(5)$ & $\mathrm{C}(40)-\mathrm{C}(39)-\mathrm{C}(38)$ & $108.2(3)$ \\
\hline
\end{tabular}




\begin{tabular}{|c|c|c|c|}
\hline$C(40)-C(39)-C(44)$ & $127.0(4)$ & $\mathrm{C}(49)-\mathrm{C}(50)-\mathrm{C}(51)$ & $108.1(4)$ \\
\hline $\mathrm{C}(38)-\mathrm{C}(39)-\mathrm{C}(44)$ & $124.7(4)$ & $\mathrm{C}(49)-\mathrm{C}(50)-\mathrm{C}(55)$ & $125.8(4)$ \\
\hline $\mathrm{C}(40)-\mathrm{C}(39)-\mathrm{U}(2)$ & $75.9(2)$ & $\mathrm{C}(51)-\mathrm{C}(50)-\mathrm{C}(55)$ & $126.1(4)$ \\
\hline $\mathrm{C}(38)-\mathrm{C}(39)-\mathrm{U}(2)$ & $75.3(2)$ & $\mathrm{C}(49)-\mathrm{C}(50)-\mathrm{U}(2)$ & $74.7(2)$ \\
\hline $\mathrm{C}(44)-\mathrm{C}(39)-\mathrm{U}(2)$ & $118.2(3)$ & $\mathrm{C}(51)-\mathrm{C}(50)-\mathrm{U}(2)$ & $75.5(2)$ \\
\hline $\mathrm{C}(39)-\mathrm{C}(40)-\mathrm{C}(41)$ & $107.8(3)$ & $\mathrm{C}(55)-\mathrm{C}(50)-\mathrm{U}(2)$ & $118.4(3)$ \\
\hline$C(39)-C(40)-C(45)$ & $126.4(4)$ & $\mathrm{C}(50)-\mathrm{C}(51)-\mathrm{C}(47)$ & $107.7(4)$ \\
\hline$C(41)-C(40)-C(45)$ & $125.0(4)$ & $\mathrm{C}(50)-\mathrm{C}(51)-\mathrm{C}(56)$ & $127.1(5)$ \\
\hline $\mathrm{C}(39)-\mathrm{C}(40)-\mathrm{U}(2)$ & $74.3(2)$ & $\mathrm{C}(47)-\mathrm{C}(51)-\mathrm{C}(56)$ & $124.6(5)$ \\
\hline $\mathrm{C}(41)-\mathrm{C}(40)-\mathrm{U}(2)$ & $75.0(2)$ & $\mathrm{C}(50)-\mathrm{C}(51)-\mathrm{U}(2)$ & $74.8(2)$ \\
\hline $\mathrm{C}(45)-\mathrm{C}(40)-\mathrm{U}(2)$ & $124.8(3)$ & $\mathrm{C}(47)-\mathrm{C}(51)-\mathrm{U}(2)$ & $74.5(2)$ \\
\hline $\mathrm{C}(37)-\mathrm{C}(41)-\mathrm{C}(40)$ & $107.6(3)$ & $\mathrm{C}(56)-\mathrm{C}(51)-\mathrm{U}(2)$ & $123.2(3)$ \\
\hline $\mathrm{C}(37)-\mathrm{C}(41)-\mathrm{C}(46)$ & $126.8(4)$ & $\mathrm{N}(3)-\mathrm{C}(57)-\mathrm{C}(59)$ & 121.1(3) \\
\hline $\mathrm{C}(40)-\mathrm{C}(41)-\mathrm{C}(46)$ & $125.4(4)$ & $\mathrm{N}(3)-\mathrm{C}(57)-\mathrm{C}(58)$ & $122.8(4)$ \\
\hline $\mathrm{C}(37)-\mathrm{C}(41)-\mathrm{U}(2)$ & $74.8(2)$ & $\mathrm{C}(59)-\mathrm{C}(57)-\mathrm{C}(58)$ & 116.1(3) \\
\hline $\mathrm{C}(40)-\mathrm{C}(41)-\mathrm{U}(2)$ & $75.1(2)$ & $\mathrm{C}(64)-\mathrm{C}(59)-\mathrm{C}(60)$ & $113.7(4)$ \\
\hline $\mathrm{C}(46)-\mathrm{C}(41)-\mathrm{U}(2)$ & $120.8(3)$ & $C(64)-C(59)-C(57)$ & $123.3(3)$ \\
\hline $\mathrm{C}(51)-\mathrm{C}(47)-\mathrm{C}(48)$ & $108.1(4)$ & $\mathrm{C}(60)-\mathrm{C}(59)-\mathrm{C}(57)$ & $122.8(4)$ \\
\hline$C(51)-C(47)-C(52)$ & $126.3(5)$ & $\mathrm{F}(5)-\mathrm{C}(60)-\mathrm{C}(61)$ & $118.0(4)$ \\
\hline $\mathrm{C}(48)-\mathrm{C}(47)-\mathrm{C}(52)$ & $125.6(5)$ & $\mathrm{F}(5)-\mathrm{C}(60)-\mathrm{C}(59)$ & $118.2(4)$ \\
\hline $\mathrm{C}(51)-\mathrm{C}(47)-\mathrm{U}(2)$ & $75.7(2)$ & $\mathrm{C}(61)-\mathrm{C}(60)-\mathrm{C}(59)$ & $123.8(4)$ \\
\hline $\mathrm{C}(48)-\mathrm{C}(47)-\mathrm{U}(2)$ & $74.8(2)$ & $\mathrm{C}(60)-\mathrm{C}(61)-\mathrm{C}(62)$ & $118.6(4)$ \\
\hline $\mathrm{C}(52)-\mathrm{C}(47)-\mathrm{U}(2)$ & $116.4(3)$ & $\mathrm{C}(61)-\mathrm{C}(62)-\mathrm{C}(63)$ & $120.8(4)$ \\
\hline $\mathrm{C}(49)-\mathrm{C}(48)-\mathrm{C}(47)$ & $107.5(4)$ & $\mathrm{C}(64)-\mathrm{C}(63)-\mathrm{C}(62)$ & $117.4(4)$ \\
\hline$C(49)-C(48)-C(53)$ & $124.4(5)$ & $\mathrm{C}(63)-\mathrm{C}(64)-\mathrm{F}(6)$ & $117.0(4)$ \\
\hline$C(47)-C(48)-C(53)$ & $128.0(5)$ & $\mathrm{C}(63)-\mathrm{C}(64)-\mathrm{C}(59)$ & $125.7(4)$ \\
\hline $\mathrm{C}(49)-\mathrm{C}(48)-\mathrm{U}(2)$ & $75.2(2)$ & $\mathrm{F}(6)-\mathrm{C}(64)-\mathrm{C}(59)$ & $117.3(4)$ \\
\hline $\mathrm{C}(47)-\mathrm{C}(48)-\mathrm{U}(2)$ & $75.2(2)$ & $\mathrm{N}(4)-\mathrm{C}(65)-\mathrm{C}(67)$ & $119.4(4)$ \\
\hline $\mathrm{C}(53)-\mathrm{C}(48)-\mathrm{U}(2)$ & $118.9(3)$ & $\mathrm{N}(4)-\mathrm{C}(65)-\mathrm{C}(66)$ & $123.6(4)$ \\
\hline $\mathrm{C}(50)-\mathrm{C}(49)-\mathrm{C}(48)$ & $108.6(4)$ & $\mathrm{C}(67)-\mathrm{C}(65)-\mathrm{C}(66)$ & $117.0(4)$ \\
\hline$C(50)-C(49)-C(54)$ & $126.7(5)$ & $\mathrm{C}(72)-\mathrm{C}(67)-\mathrm{C}(68)$ & $114.9(4)$ \\
\hline $\mathrm{C}(48)-\mathrm{C}(49)-\mathrm{C}(54)$ & $124.4(5)$ & $\mathrm{C}(72)-\mathrm{C}(67)-\mathrm{C}(65)$ & $121.8(4)$ \\
\hline $\mathrm{C}(50)-\mathrm{C}(49)-\mathrm{U}(2)$ & $75.6(2)$ & $\mathrm{C}(68)-\mathrm{C}(67)-\mathrm{C}(65)$ & $123.3(4)$ \\
\hline $\mathrm{C}(48)-\mathrm{C}(49)-\mathrm{U}(2)$ & $75.1(2)$ & $\mathrm{F}(7)-\mathrm{C}(68)-\mathrm{C}(69)$ & $119.1(5)$ \\
\hline $\mathrm{C}(54)-\mathrm{C}(49)-\mathrm{U}(2)$ & $120.1(3)$ & $\mathrm{F}(7)-\mathrm{C}(68)-\mathrm{C}(67)$ & $117.0(4)$ \\
\hline
\end{tabular}




\begin{tabular}{|l|lr|}
\hline & $\mathrm{C}(69)-\mathrm{C}(68)-\mathrm{C}(67)$ & $123.8(6)$ \\
& $\mathrm{C}(68)-\mathrm{C}(69)-\mathrm{C}(70)$ & $118.2(5)$ \\
& $\mathrm{C}(71)-\mathrm{C}(70)-\mathrm{C}(69)$ & $120.4(5)$ \\
& $\mathrm{C}(70)-\mathrm{C}(71)-\mathrm{C}(72)$ & $119.3(6)$ \\
& $\mathrm{F}(8)-\mathrm{C}(72)-\mathrm{C}(67)$ & $118.9(4)$ \\
& $\mathrm{F}(8)-\mathrm{C}(72)-\mathrm{C}(71)$ & $117.7(5)$ \\
& $\mathrm{C}(67)-\mathrm{C}(72)-\mathrm{C}(71)$ & $123.3(5)$ \\
\hline
\end{tabular}


Table S9: Crystal Data and Structure Refinement for $\left(\mathrm{C}_{5} \mathrm{Me}_{5}\right)_{2} \mathrm{U}\left[-\mathrm{N}=\mathrm{C}\left(\mathrm{CH}_{3}\right)\left(2,4,6-\mathrm{F}_{3}-\mathrm{C}_{6} \mathrm{H}_{3}\right)\right]_{2}$ (9).

\begin{tabular}{|c|c|}
\hline Empirical formula & $\mathrm{C}_{36} \mathrm{H}_{40} \mathrm{~F}_{6} \mathrm{~N}_{2} \mathrm{U}$ \\
\hline Formula weight & 852.73 \\
\hline Temperature & $141(2) \mathrm{K}$ \\
\hline Wavelength & $0.71073 \AA$ \\
\hline Crystal system & monoclinic \\
\hline Space group & $\mathrm{C} 2 / \mathrm{c}$ \\
\hline \multirow[t]{3}{*}{ Unit cell dimensions } & $\mathrm{a}=13.5252(5) \AA$ \\
\hline & $\mathrm{b}=14.5744(5) \AA$ \\
\hline & $\mathrm{c}=17.1466(6) \AA$ \\
\hline Volume & $3358.7(2) \AA^{3}$ \\
\hline $\mathrm{Z}$ & 4 \\
\hline Density (calculated) & $1.686 \mathrm{Mg} / \mathrm{m}^{3}$ \\
\hline Absorption coefficient & $4.893 \mathrm{~mm}^{-1}$ \\
\hline $\mathrm{F}(000)$ & 1664 \\
\hline Crystal size & $0.28 \times 0.10 \times 0.08 \mathrm{~mm}^{3}$ \\
\hline$\Theta$ Range for data collection & 2.06 to $28.98^{\circ}$ \\
\hline Index ranges & $-18 \leq \mathrm{h} \leq 18,-19 \leq \mathrm{k} \leq 19,-22 \leq 1 \leq 23$ \\
\hline Reflections collected & 16395 \\
\hline Independent reflections & $4131[\mathrm{R}(\mathrm{int})=0.0323]$ \\
\hline Completeness to $\Theta=25.00^{\circ}$ & $100.0 \%$ \\
\hline Max. and min. transmission & 0.6956 and 0.3412 \\
\hline Refinement method & Full-matrix least-squares on $\mathrm{F}^{2}$ \\
\hline Data / restraints / parameters & $4131 / 0 / 210$ \\
\hline Goodness-of-fit on $\mathrm{F}^{2}$ & 1.118 \\
\hline Final $R$ indices $[\mathrm{I}>2 \sigma(\mathrm{I})]$ & $\mathrm{R} 1=0.0197, \mathrm{wR} 2=0.0492$ \\
\hline $\mathrm{R}$ indices (all data) & $\mathrm{R} 1=0.0223, \mathrm{wR} 2=0.0501$ \\
\hline Largest diff. peak and hole & 1.044 and -0.527 e..$\AA^{-3}$ \\
\hline
\end{tabular}


Table S10: Bond Distances $[\AA]$ and Angles $\left[^{\circ}\right]$ for $\left(\mathrm{C}_{5} \mathrm{Me}_{5}\right)_{2} \mathrm{U}\left[-\mathrm{N}=\mathrm{C}\left(\mathrm{CH}_{3}\right)\left(2,4,6-\mathrm{F}_{3}-\mathrm{C}_{6} \mathrm{H}_{3}\right)\right]_{2}(\mathbf{9})$.

\begin{tabular}{|c|c|c|c|}
\hline $\mathrm{U}(1)-\mathrm{N}(1) \# 1$ & $2.193(2)$ & $\mathrm{N}(1) \# 1-\mathrm{U}(1)-\mathrm{N}(1)$ & $105.49(11)$ \\
\hline $\mathrm{U}(1)-\mathrm{N}(1)$ & $2.193(2)$ & $\mathrm{N}(1) \# 1-\mathrm{U}(1)-\mathrm{C}(3) \# 1$ & $79.82(7)$ \\
\hline $\mathrm{U}(1)-\mathrm{C}(3) \# 1$ & $2.739(2)$ & $\mathrm{N}(1)-\mathrm{U}(1)-\mathrm{C}(3) \# 1$ & $124.62(7)$ \\
\hline $\mathrm{U}(1)-\mathrm{C}(3)$ & $2.739(2)$ & $\mathrm{N}(1) \# 1-\mathrm{U}(1)-\mathrm{C}(3)$ & $124.62(7)$ \\
\hline $\mathrm{U}(1)-\mathrm{C}(5)$ & $2.740(2)$ & $\mathrm{N}(1)-\mathrm{U}(1)-\mathrm{C}(3)$ & $79.82(7)$ \\
\hline $\mathrm{U}(1)-\mathrm{C}(5) \# 1$ & $2.740(2)$ & $\mathrm{C}(3) \# 1-\mathrm{U}(1)-\mathrm{C}(3)$ & $142.28(10)$ \\
\hline $\mathrm{U}(1)-\mathrm{C}(1)$ & $2.742(2)$ & $\mathrm{N}(1) \# 1-\mathrm{U}(1)-\mathrm{C}(5)$ & $77.69(7)$ \\
\hline $\mathrm{U}(1)-\mathrm{C}(1) \# 1$ & $2.742(2)$ & $\mathrm{N}(1)-\mathrm{U}(1)-\mathrm{C}(5)$ & $107.24(7)$ \\
\hline $\mathrm{U}(1)-\mathrm{C}(4) \# 1$ & $2.747(2)$ & $C(3) \# 1-U(1)-C(5)$ & $127.24(7)$ \\
\hline $\mathrm{U}(1)-\mathrm{C}(4)$ & $2.747(2)$ & $\mathrm{C}(3)-\mathrm{U}(1)-\mathrm{C}(5)$ & $49.49(7)$ \\
\hline $\mathrm{U}(1)-\mathrm{C}(2) \# 1$ & $2.747(2)$ & $\mathrm{N}(1) \# 1-\mathrm{U}(1)-\mathrm{C}(5) \# 1$ & $107.24(7)$ \\
\hline $\mathrm{U}(1)-\mathrm{C}(2)$ & $2.747(2)$ & $\mathrm{N}(1)-\mathrm{U}(1)-\mathrm{C}(5) \# 1$ & $77.69(7)$ \\
\hline $\mathrm{F}(1)-\mathrm{C}(14)$ & $1.354(3)$ & $\mathrm{C}(3) \# 1-\mathrm{U}(1)-\mathrm{C}(5) \# 1$ & $49.49(7)$ \\
\hline $\mathrm{F}(2)-\mathrm{C}(16)$ & $1.354(3)$ & $\mathrm{C}(3)-\mathrm{U}(1)-\mathrm{C}(5) \# 1$ & $127.24(7)$ \\
\hline $\mathrm{F}(3)-\mathrm{C}(18)$ & $1.350(3)$ & $C(5)-U(1)-C(5) \# 1$ & $172.13(10)$ \\
\hline $\mathrm{N}(1)-\mathrm{C}(11)$ & $1.257(3)$ & $\mathrm{N}(1) \# 1-\mathrm{U}(1)-\mathrm{C}(1)$ & $93.81(7)$ \\
\hline$C(1)-C(5)$ & $1.419(3)$ & $\mathrm{N}(1)-\mathrm{U}(1)-\mathrm{C}(1)$ & $127.06(7)$ \\
\hline $\mathrm{C}(1)-\mathrm{C}(2)$ & $1.419(3)$ & $\mathrm{C}(3) \# 1-\mathrm{U}(1)-\mathrm{C}(1)$ & $106.90(7)$ \\
\hline$C(1)-C(6)$ & $1.502(3)$ & $\mathrm{C}(3)-\mathrm{U}(1)-\mathrm{C}(1)$ & $49.61(7)$ \\
\hline$C(2)-C(3)$ & $1.426(3)$ & $\mathrm{C}(5)-\mathrm{U}(1)-\mathrm{C}(1)$ & $30.00(7)$ \\
\hline$C(2)-C(7)$ & $1.508(3)$ & $C(5) \# 1-U(1)-C(1)$ & $142.20(7)$ \\
\hline $\mathrm{C}(3)-\mathrm{C}(4)$ & $1.426(3)$ & $\mathrm{N}(1) \# 1-\mathrm{U}(1)-\mathrm{C}(1) \# 1$ & $127.06(7)$ \\
\hline $\mathrm{C}(3)-\mathrm{C}(8)$ & $1.503(3)$ & $\mathrm{N}(1)-\mathrm{U}(1)-\mathrm{C}(1) \# 1$ & $93.81(7)$ \\
\hline $\mathrm{C}(4)-\mathrm{C}(5)$ & $1.424(3)$ & $\mathrm{C}(3) \# 1-\mathrm{U}(1)-\mathrm{C}(1) \# 1$ & $49.61(7)$ \\
\hline $\mathrm{C}(4)-\mathrm{C}(9)$ & $1.501(3)$ & $\mathrm{C}(3)-\mathrm{U}(1)-\mathrm{C}(1) \# 1$ & $106.90(7)$ \\
\hline$C(5)-C(10)$ & $1.510(3)$ & $\mathrm{C}(5)-\mathrm{U}(1)-\mathrm{C}(1) \# 1$ & $142.20(7)$ \\
\hline $\mathrm{C}(11)-\mathrm{C}(13)$ & $1.518(3)$ & $\mathrm{C}(5) \# 1-\mathrm{U}(1)-\mathrm{C}(1) \# 1$ & $30.00(7)$ \\
\hline $\mathrm{C}(11)-\mathrm{C}(12)$ & $1.520(3)$ & $\mathrm{C}(1)-\mathrm{U}(1)-\mathrm{C}(1) \# 1$ & $112.89(10)$ \\
\hline$C(13)-C(14)$ & $1.388(3)$ & $\mathrm{N}(1) \# 1-\mathrm{U}(1)-\mathrm{C}(4) \# 1$ & $79.20(7)$ \\
\hline$C(13)-C(18)$ & $1.393(3)$ & $\mathrm{N}(1)-\mathrm{U}(1)-\mathrm{C}(4) \# 1$ & $95.39(7)$ \\
\hline $\mathrm{C}(14)-\mathrm{C}(15)$ & $1.373(3)$ & $\mathrm{C}(3) \# 1-\mathrm{U}(1)-\mathrm{C}(4) \# 1$ & $30.14(7)$ \\
\hline$C(15)-C(16)$ & $1.377(4)$ & $\mathrm{C}(3)-\mathrm{U}(1)-\mathrm{C}(4) \# 1$ & $156.17(7)$ \\
\hline$C(16)-C(17)$ & $1.375(4)$ & $\mathrm{C}(5)-\mathrm{U}(1)-\mathrm{C}(4) \# 1$ & $151.15(7)$ \\
\hline $\mathrm{C}(17)-\mathrm{C}(18)$ & $1.376(3)$ & $\mathrm{C}(5) \# 1-\mathrm{U}(1)-\mathrm{C}(4) \# 1$ & $30.09(7)$ \\
\hline
\end{tabular}




\begin{tabular}{|c|c|c|c|}
\hline $\mathrm{C}(1)-\mathrm{U}(1)-\mathrm{C}(4) \# 1$ & $136.98(7)$ & $\mathrm{C}(2)-\mathrm{C}(1)-\mathrm{C}(6)$ & $127.2(2)$ \\
\hline $\mathrm{C}(1) \# 1-\mathrm{U}(1)-\mathrm{C}(4) \# 1$ & $49.78(7)$ & $\mathrm{C}(5)-\mathrm{C}(1)-\mathrm{U}(1)$ & $74.92(13)$ \\
\hline $\mathrm{N}(1) \# 1-\mathrm{U}(1)-\mathrm{C}(4)$ & $95.39(7)$ & $\mathrm{C}(2)-\mathrm{C}(1)-\mathrm{U}(1)$ & $75.23(12)$ \\
\hline $\mathrm{N}(1)-\mathrm{U}(1)-\mathrm{C}(4)$ & $79.20(7)$ & $\mathrm{C}(6)-\mathrm{C}(1)-\mathrm{U}(1)$ & $118.93(15)$ \\
\hline $\mathrm{C}(3) \# 1-\mathrm{U}(1)-\mathrm{C}(4)$ & $156.17(7)$ & $\mathrm{C}(1)-\mathrm{C}(2)-\mathrm{C}(3)$ & $107.8(2)$ \\
\hline $\mathrm{C}(3)-\mathrm{U}(1)-\mathrm{C}(4)$ & $30.14(7)$ & $\mathrm{C}(1)-\mathrm{C}(2)-\mathrm{C}(7)$ & $127.9(2)$ \\
\hline $\mathrm{C}(5)-\mathrm{U}(1)-\mathrm{C}(4)$ & $30.09(7)$ & $\mathrm{C}(3)-\mathrm{C}(2)-\mathrm{C}(7)$ & $123.7(2)$ \\
\hline $\mathrm{C}(5) \# 1-\mathrm{U}(1)-\mathrm{C}(4)$ & $151.15(7)$ & $\mathrm{C}(1)-\mathrm{C}(2)-\mathrm{U}(1)$ & $74.81(13)$ \\
\hline $\mathrm{C}(1)-\mathrm{U}(1)-\mathrm{C}(4)$ & $49.78(7)$ & $\mathrm{C}(3)-\mathrm{C}(2)-\mathrm{U}(1)$ & $74.61(13)$ \\
\hline $\mathrm{C}(1) \# 1-\mathrm{U}(1)-\mathrm{C}(4)$ & $136.98(7)$ & $\mathrm{C}(7)-\mathrm{C}(2)-\mathrm{U}(1)$ & $123.00(15)$ \\
\hline $\mathrm{C}(4) \# 1-\mathrm{U}(1)-\mathrm{C}(4)$ & $171.14(10)$ & $\mathrm{C}(4)-\mathrm{C}(3)-\mathrm{C}(2)$ & $108.4(2)$ \\
\hline $\mathrm{N}(1) \# 1-\mathrm{U}(1)-\mathrm{C}(2) \# 1$ & $108.15(7)$ & $\mathrm{C}(4)-\mathrm{C}(3)-\mathrm{C}(8)$ & $126.6(2)$ \\
\hline $\mathrm{N}(1)-\mathrm{U}(1)-\mathrm{C}(2) \# 1$ & $123.33(7)$ & $\mathrm{C}(2)-\mathrm{C}(3)-\mathrm{C}(8)$ & $124.9(2)$ \\
\hline $\mathrm{C}(3) \# 1-\mathrm{U}(1)-\mathrm{C}(2) \# 1$ & $30.13(7)$ & $\mathrm{C}(4)-\mathrm{C}(3)-\mathrm{U}(1)$ & $75.25(13)$ \\
\hline $\mathrm{C}(3)-\mathrm{U}(1)-\mathrm{C}(2) \# 1$ & $113.90(7)$ & $\mathrm{C}(2)-\mathrm{C}(3)-\mathrm{U}(1)$ & $75.26(13)$ \\
\hline $\mathrm{C}(5)-\mathrm{U}(1)-\mathrm{C}(2) \# 1$ & $123.54(7)$ & $\mathrm{C}(8)-\mathrm{C}(3)-\mathrm{U}(1)$ & $119.79(15)$ \\
\hline $\mathrm{C}(5) \# 1-\mathrm{U}(1)-\mathrm{C}(2) \# 1$ & $49.45(7)$ & $C(5)-C(4)-C(3)$ & 107.1(2) \\
\hline $\mathrm{C}(1)-\mathrm{U}(1)-\mathrm{C}(2) \# 1$ & $94.60(7)$ & $\mathrm{C}(5)-\mathrm{C}(4)-\mathrm{C}(9)$ & $125.9(2)$ \\
\hline $\mathrm{C}(1) \# 1-\mathrm{U}(1)-\mathrm{C}(2) \# 1$ & $29.96(6)$ & $C(3)-C(4)-C(9)$ & $126.8(2)$ \\
\hline $\mathrm{C}(4) \# 1-\mathrm{U}(1)-\mathrm{C}(2) \# 1$ & $49.79(7)$ & $\mathrm{C}(5)-\mathrm{C}(4)-\mathrm{U}(1)$ & $74.68(14)$ \\
\hline $\mathrm{C}(4)-\mathrm{U}(1)-\mathrm{C}(2) \# 1$ & $139.06(7)$ & $\mathrm{C}(3)-\mathrm{C}(4)-\mathrm{U}(1)$ & $74.61(13)$ \\
\hline N(1)\#1-U(1)-C(2) & $123.33(7)$ & $\mathrm{C}(9)-\mathrm{C}(4)-\mathrm{U}(1)$ & $120.72(15)$ \\
\hline $\mathrm{N}(1)-\mathrm{U}(1)-\mathrm{C}(2)$ & $108.15(7)$ & $C(1)-C(5)-C(4)$ & $108.7(2)$ \\
\hline $\mathrm{C}(3) \# 1-\mathrm{U}(1)-\mathrm{C}(2)$ & $113.90(7)$ & $C(1)-C(5)-C(10)$ & $125.3(2)$ \\
\hline $\mathrm{C}(3)-\mathrm{U}(1)-\mathrm{C}(2)$ & $30.13(7)$ & $C(4)-C(5)-C(10)$ & $125.6(2)$ \\
\hline $\mathrm{C}(5)-\mathrm{U}(1)-\mathrm{C}(2)$ & $49.45(7)$ & $\mathrm{C}(1)-\mathrm{C}(5)-\mathrm{U}(1)$ & $75.08(13)$ \\
\hline $\mathrm{C}(5) \# 1-\mathrm{U}(1)-\mathrm{C}(2)$ & $123.54(7)$ & $\mathrm{C}(4)-\mathrm{C}(5)-\mathrm{U}(1)$ & $75.24(14)$ \\
\hline $\mathrm{C}(1)-\mathrm{U}(1)-\mathrm{C}(2)$ & $29.96(6)$ & $\mathrm{C}(10)-\mathrm{C}(5)-\mathrm{U}(1)$ & $121.49(15)$ \\
\hline $\mathrm{C}(1) \# 1-\mathrm{U}(1)-\mathrm{C}(2)$ & $94.60(7)$ & $\mathrm{N}(1)-\mathrm{C}(11)-\mathrm{C}(13)$ & $121.2(2)$ \\
\hline $\mathrm{C}(4) \# 1-\mathrm{U}(1)-\mathrm{C}(2)$ & $139.06(7)$ & $\mathrm{N}(1)-\mathrm{C}(11)-\mathrm{C}(12)$ & $124.1(2)$ \\
\hline $\mathrm{C}(4)-\mathrm{U}(1)-\mathrm{C}(2)$ & $49.79(7)$ & $\mathrm{C}(13)-\mathrm{C}(11)-\mathrm{C}(12)$ & $114.7(2)$ \\
\hline $\mathrm{C}(2) \# 1-\mathrm{U}(1)-\mathrm{C}(2)$ & $89.34(10)$ & $C(14)-C(13)-C(18)$ & $114.3(2)$ \\
\hline $\mathrm{C}(11)-\mathrm{N}(1)-\mathrm{U}(1)$ & $176.78(19)$ & $\mathrm{C}(14)-\mathrm{C}(13)-\mathrm{C}(11)$ & $123.4(2)$ \\
\hline$C(5)-C(1)-C(2)$ & $108.0(2)$ & $C(18)-C(13)-C(11)$ & $122.3(2)$ \\
\hline$C(5)-C(1)-C(6)$ & $124.8(2)$ & $\mathrm{F}(1)-\mathrm{C}(14)-\mathrm{C}(15)$ & $117.9(2)$ \\
\hline
\end{tabular}




\begin{tabular}{|ll|l|}
\hline$F(1)-C(14)-C(13)$ & $117.7(2)$ & \\
$C(15)-C(14)-C(13)$ & $124.5(2)$ & \\
$C(14)-C(15)-C(16)$ & $117.0(2)$ & \\
F(2)-C(16)-C(17) & $118.5(2)$ & \\
F(2)-C(16)-C(15) & $118.5(2)$ & \\
C(17)-C(16)-C(15) & $122.9(2)$ & \\
C(18)-C(17)-C(16) & $116.6(2)$ & \\
F(3)-C(18)-C(17) & $117.1(2)$ & \\
F(3)-C(18)-C(13) & $118.3(2)$ & \\
C(17)-C(18)-C(13) & $124.6(2)$ & \\
\hline
\end{tabular}


Table S11: Crystal Data and Structure Refinement for $\left(\mathrm{C}_{5} \mathrm{Me}_{5}\right)_{2} \mathrm{U}\left[-\mathrm{N}=\mathrm{C}\left(\mathrm{CH}_{3}\right)\left(3,4,5-\mathrm{F}_{3}-\mathrm{C}_{6} \mathrm{H}_{3}\right)\right]_{2}(\mathbf{1 0})$.

\begin{tabular}{|c|c|}
\hline Empirical formula & $\mathrm{C}_{36} \mathrm{H}_{40} \mathrm{~F}_{6} \mathrm{~N}_{2} \mathrm{U}$ \\
\hline Formula weight & 852.73 \\
\hline Temperature & $141(2) \mathrm{K}$ \\
\hline Wavelength & $0.71073 \AA$ \\
\hline Crystal system & monoclinic \\
\hline Space group & $\mathrm{C} 2 / \mathrm{c}$ \\
\hline \multirow[t]{3}{*}{ Unit cell dimensions } & $a=12.9529(6) \AA$ \\
\hline & $\mathrm{b}=13.8105(6) \AA$ \\
\hline & $\mathrm{c}=18.5471(8) \AA$ \\
\hline Volume & $3269.6(3) \AA^{3}$ \\
\hline $\mathrm{Z}$ & 4 \\
\hline Density (calculated) & $1.732 \mathrm{Mg} / \mathrm{m}^{3}$ \\
\hline Absorption coefficient & $5.027 \mathrm{~mm}^{-1}$ \\
\hline $\mathrm{F}(000)$ & 1664 \\
\hline Crystal size & $0.20 \times 0.16 \times 0.10 \mathrm{~mm}^{3}$ \\
\hline$\Theta$ Range for data collection & 2.17 to $28.99^{\circ}$ \\
\hline Index ranges & $-17 \leq \mathrm{h} \leq 16,-18 \leq \mathrm{k} \leq 18,-24 \leq 1 \leq 25$ \\
\hline Reflections collected & 18097 \\
\hline Independent reflections & $4024[\mathrm{R}($ int $)=0.0243]$ \\
\hline Completeness to $\Theta=28.99^{\circ}$ & $92.8 \%$ \\
\hline Max. and min. transmission & 0.6333 and 0.4330 \\
\hline Refinement method & Full-matrix least-squares on $\mathrm{F}^{2}$ \\
\hline Data / restraints / parameters & 4024 / 0 / 204 \\
\hline Goodness-of-fit on $\mathrm{F}^{2}$ & 1.346 \\
\hline Final $R$ indices $[I>2 \sigma(I)]$ & $\mathrm{R} 1=0.0182, \mathrm{wR} 2=0.0495$ \\
\hline $\mathrm{R}$ indices (all data) & $\mathrm{R} 1=0.0192, \mathrm{wR} 2=0.0498$ \\
\hline Largest diff. peak and hole & 0.869 and -0.504 e. $. \AA-3$ \\
\hline
\end{tabular}


Table S12: Bond Distances $[\AA]$ and Angles $\left[{ }^{\circ}\right]$ for $\left(\mathrm{C}_{5} \mathrm{Me}_{5}\right)_{2} \mathrm{U}\left[-\mathrm{N}=\mathrm{C}\left(\mathrm{CH}_{3}\right)\left(3,4,5-\mathrm{F}_{3}-\mathrm{C}_{6} \mathrm{H}_{3}\right)\right]_{2}(\mathbf{1 0})$.

\begin{tabular}{|c|c|c|c|}
\hline $\mathrm{U}(1)-\mathrm{N}(1)$ & $2.1974(18)$ & $\mathrm{N}(1)-\mathrm{U}(1)-\mathrm{N}(1) \# 1$ & $111.83(10)$ \\
\hline $\mathrm{U}(1)-\mathrm{N}(1) \# 1$ & $2.1974(18)$ & $\mathrm{N}(1)-\mathrm{U}(1)-\mathrm{C}(3) \# 1$ & $119.58(7)$ \\
\hline $\mathrm{U}(1)-\mathrm{C}(3) \# 1$ & $2.735(2)$ & $\mathrm{N}(1) \# 1-\mathrm{U}(1)-\mathrm{C}(3) \# 1$ & $106.49(7)$ \\
\hline $\mathrm{U}(1)-\mathrm{C}(3)$ & $2.735(2)$ & $\mathrm{N}(1)-\mathrm{U}(1)-\mathrm{C}(3)$ & $106.49(7)$ \\
\hline $\mathrm{U}(1)-\mathrm{C}(4) \# 1$ & $2.738(2)$ & $\mathrm{N}(1) \# 1-\mathrm{U}(1)-\mathrm{C}(3)$ & $119.58(7)$ \\
\hline $\mathrm{U}(1)-\mathrm{C}(4)$ & $2.738(2)$ & $\mathrm{C}(3) \# 1-\mathrm{U}(1)-\mathrm{C}(3)$ & $92.16(9)$ \\
\hline $\mathrm{U}(1)-\mathrm{C}(2) \# 1$ & $2.743(2)$ & $\mathrm{N}(1)-\mathrm{U}(1)-\mathrm{C}(4) \# 1$ & $123.41(7)$ \\
\hline $\mathrm{U}(1)-\mathrm{C}(2)$ & $2.743(2)$ & $\mathrm{N}(1) \# 1-\mathrm{U}(1)-\mathrm{C}(4) \# 1$ & $78.46(7)$ \\
\hline $\mathrm{U}(1)-\mathrm{C}(5)$ & $2.745(2)$ & $\mathrm{C}(3) \# 1-\mathrm{U}(1)-\mathrm{C}(4) \# 1$ & $29.97(7)$ \\
\hline $\mathrm{U}(1)-\mathrm{C}(5) \# 1$ & $2.745(2)$ & $\mathrm{C}(3)-\mathrm{U}(1)-\mathrm{C}(4) \# 1$ & $115.62(7)$ \\
\hline $\mathrm{U}(1)-\mathrm{C}(1)$ & $2.753(2)$ & $\mathrm{N}(1)-\mathrm{U}(1)-\mathrm{C}(4)$ & $78.46(7)$ \\
\hline $\mathrm{U}(1)-\mathrm{C}(1) \# 1$ & $2.753(2)$ & $\mathrm{N}(1) \# 1-\mathrm{U}(1)-\mathrm{C}(4)$ & $123.41(7)$ \\
\hline $\mathrm{F}(1)-\mathrm{C}(15)$ & $1.349(3)$ & $\mathrm{C}(3) \# 1-\mathrm{U}(1)-\mathrm{C}(4)$ & $115.62(7)$ \\
\hline $\mathrm{F}(2)-\mathrm{C}(16)$ & $1.351(3)$ & $\mathrm{C}(3)-\mathrm{U}(1)-\mathrm{C}(4)$ & $29.97(7)$ \\
\hline $\mathrm{F}(3)-\mathrm{C}(17)$ & $1.349(3)$ & C(4)\#1-U(1)-C(4) & $143.55(10)$ \\
\hline $\mathrm{N}(1)-\mathrm{C}(11)$ & $1.264(3)$ & $\mathrm{N}(1)-\mathrm{U}(1)-\mathrm{C}(2) \# 1$ & $89.79(7)$ \\
\hline$C(1)-C(5)$ & $1.419(3)$ & $\mathrm{N}(1) \# 1-\mathrm{U}(1)-\mathrm{C}(2) \# 1$ & $126.00(7)$ \\
\hline$C(1)-C(2)$ & $1.432(3)$ & $\mathrm{C}(3) \# 1-\mathrm{U}(1)-\mathrm{C}(2) \# 1$ & $30.01(6)$ \\
\hline$C(1)-C(6)$ & $1.502(3)$ & $\mathrm{C}(3)-\mathrm{U}(1)-\mathrm{C}(2) \# 1$ & $98.22(7)$ \\
\hline $\mathrm{C}(2)-\mathrm{C}(3)$ & $1.418(3)$ & $\mathrm{C}(4) \# 1-\mathrm{U}(1)-\mathrm{C}(2) \# 1$ & $49.59(6)$ \\
\hline$C(2)-C(7)$ & $1.499(3)$ & $\mathrm{C}(4)-\mathrm{U}(1)-\mathrm{C}(2) \# 1$ & $108.80(7)$ \\
\hline$C(3)-C(4)$ & $1.415(3)$ & $\mathrm{N}(1)-\mathrm{U}(1)-\mathrm{C}(2)$ & $126.00(7)$ \\
\hline $\mathrm{C}(3)-\mathrm{C}(8)$ & $1.517(3)$ & $\mathrm{N}(1) \# 1-\mathrm{U}(1)-\mathrm{C}(2)$ & $89.79(7)$ \\
\hline $\mathrm{C}(4)-\mathrm{C}(5)$ & $1.422(3)$ & $\mathrm{C}(3) \# 1-\mathrm{U}(1)-\mathrm{C}(2)$ & $98.22(6)$ \\
\hline $\mathrm{C}(4)-\mathrm{C}(9)$ & $1.498(3)$ & $\mathrm{C}(3)-\mathrm{U}(1)-\mathrm{C}(2)$ & $30.01(6)$ \\
\hline$C(5)-C(10)$ & $1.509(3)$ & $\mathrm{C}(4) \# 1-\mathrm{U}(1)-\mathrm{C}(2)$ & $108.80(7)$ \\
\hline $\mathrm{C}(11)-\mathrm{C}(13)$ & $1.507(3)$ & $\mathrm{C}(4)-\mathrm{U}(1)-\mathrm{C}(2)$ & $49.59(6)$ \\
\hline $\mathrm{C}(11)-\mathrm{C}(12)$ & $1.524(3)$ & $\mathrm{C}(2) \# 1-\mathrm{U}(1)-\mathrm{C}(2)$ & $117.18(9)$ \\
\hline$C(13)-C(14)$ & $1.400(3)$ & $\mathrm{N}(1)-\mathrm{U}(1)-\mathrm{C}(5)$ & $78.52(7)$ \\
\hline$C(13)-C(18)$ & $1.407(3)$ & $\mathrm{N}(1) \# 1-\mathrm{U}(1)-\mathrm{C}(5)$ & $95.05(7)$ \\
\hline $\mathrm{C}(14)-\mathrm{C}(15)$ & $1.375(3)$ & $\mathrm{C}(3) \# 1-\mathrm{U}(1)-\mathrm{C}(5)$ & $141.77(7)$ \\
\hline$C(15)-C(16)$ & $1.379(4)$ & $\mathrm{C}(3)-\mathrm{U}(1)-\mathrm{C}(5)$ & $49.61(7)$ \\
\hline$C(16)-C(17)$ & $1.374(4)$ & $\mathrm{C}(4) \# 1-\mathrm{U}(1)-\mathrm{C}(5)$ & $158.04(7)$ \\
\hline $\mathrm{C}(17)-\mathrm{C}(18)$ & $1.367(3)$ & $\mathrm{C}(4)-\mathrm{U}(1)-\mathrm{C}(5)$ & $30.07(7)$ \\
\hline
\end{tabular}




\begin{tabular}{|c|c|c|c|}
\hline $\mathrm{C}(2) \# 1-\mathrm{U}(1)-\mathrm{C}(5)$ & $138.54(7)$ & $\mathrm{C}(2)-\mathrm{C}(1)-\mathrm{C}(6)$ & $126.2(2)$ \\
\hline$C(2)-U(1)-C(5)$ & $49.75(7)$ & $\mathrm{C}(5)-\mathrm{C}(1)-\mathrm{U}(1)$ & $74.73(13)$ \\
\hline $\mathrm{N}(1)-\mathrm{U}(1)-\mathrm{C}(5) \# 1$ & $95.05(7)$ & $\mathrm{C}(2)-\mathrm{C}(1)-\mathrm{U}(1)$ & $74.49(13)$ \\
\hline $\mathrm{N}(1) \# 1-\mathrm{U}(1)-\mathrm{C}(5) \# 1$ & $78.52(7)$ & $\mathrm{C}(6)-\mathrm{C}(1)-\mathrm{U}(1)$ & $122.35(15)$ \\
\hline $\mathrm{C}(3) \# 1-\mathrm{U}(1)-\mathrm{C}(5) \# 1$ & $49.61(7)$ & $\mathrm{C}(3)-\mathrm{C}(2)-\mathrm{C}(1)$ & $107.44(19)$ \\
\hline $\mathrm{C}(3)-\mathrm{U}(1)-\mathrm{C}(5) \# 1$ & $141.77(7)$ & $\mathrm{C}(3)-\mathrm{C}(2)-\mathrm{C}(7)$ & $127.2(2)$ \\
\hline $\mathrm{C}(4) \# 1-\mathrm{U}(1)-\mathrm{C}(5) \# 1$ & $30.07(7)$ & $\mathrm{C}(1)-\mathrm{C}(2)-\mathrm{C}(7)$ & $125.2(2)$ \\
\hline $\mathrm{C}(4)-\mathrm{U}(1)-\mathrm{C}(5) \# 1$ & $158.04(7)$ & $\mathrm{C}(3)-\mathrm{C}(2)-\mathrm{U}(1)$ & $74.69(12)$ \\
\hline $\mathrm{C}(2) \# 1-\mathrm{U}(1)-\mathrm{C}(5) \# 1$ & $49.75(7)$ & $\mathrm{C}(1)-\mathrm{C}(2)-\mathrm{U}(1)$ & $75.31(12)$ \\
\hline $\mathrm{C}(2)-\mathrm{U}(1)-\mathrm{C}(5) \# 1$ & $138.54(7)$ & $\mathrm{C}(7)-\mathrm{C}(2)-\mathrm{U}(1)$ & $119.77(15)$ \\
\hline $\mathrm{C}(5)-\mathrm{U}(1)-\mathrm{C}(5) \# 1$ & 168.62(9) & $\mathrm{C}(4)-\mathrm{C}(3)-\mathrm{C}(2)$ & $108.43(19)$ \\
\hline $\mathrm{N}(1)-\mathrm{U}(1)-\mathrm{C}(1)$ & $106.62(7)$ & $\mathrm{C}(4)-\mathrm{C}(3)-\mathrm{C}(8)$ & $124.7(2)$ \\
\hline $\mathrm{N}(1) \# 1-\mathrm{U}(1)-\mathrm{C}(1)$ & $75.36(7)$ & $C(2)-C(3)-C(8)$ & $126.3(2)$ \\
\hline $\mathrm{C}(3) \# 1-\mathrm{U}(1)-\mathrm{C}(1)$ & $127.47(6)$ & $\mathrm{C}(4)-\mathrm{C}(3)-\mathrm{U}(1)$ & $75.15(12)$ \\
\hline$C(3)-U(1)-C(1)$ & $49.49(6)$ & $\mathrm{C}(2)-\mathrm{C}(3)-\mathrm{U}(1)$ & $75.30(12)$ \\
\hline $\mathrm{C}(4) \# 1-\mathrm{U}(1)-\mathrm{C}(1)$ & $129.21(7)$ & $\mathrm{C}(8)-\mathrm{C}(3)-\mathrm{U}(1)$ & $122.75(15)$ \\
\hline $\mathrm{C}(4)-\mathrm{U}(1)-\mathrm{C}(1)$ & $49.40(7)$ & $C(3)-C(4)-C(5)$ & $108.22(19)$ \\
\hline $\mathrm{C}(2) \# 1-\mathrm{U}(1)-\mathrm{C}(1)$ & $146.45(6)$ & $\mathrm{C}(3)-\mathrm{C}(4)-\mathrm{C}(9)$ & $125.1(2)$ \\
\hline$C(2)-U(1)-C(1)$ & $30.20(6)$ & $C(5)-C(4)-C(9)$ & $126.4(2)$ \\
\hline $\mathrm{C}(5)-\mathrm{U}(1)-\mathrm{C}(1)$ & $29.91(7)$ & $\mathrm{C}(3)-\mathrm{C}(4)-\mathrm{U}(1)$ & $74.88(12)$ \\
\hline $\mathrm{C}(5) \# 1-\mathrm{U}(1)-\mathrm{C}(1)$ & $150.77(7)$ & $\mathrm{C}(5)-\mathrm{C}(4)-\mathrm{U}(1)$ & $75.24(12)$ \\
\hline $\mathrm{N}(1)-\mathrm{U}(1)-\mathrm{C}(1) \# 1$ & $75.36(7)$ & $\mathrm{C}(9)-\mathrm{C}(4)-\mathrm{U}(1)$ & $120.79(15)$ \\
\hline $\mathrm{N}(1) \# 1-\mathrm{U}(1)-\mathrm{C}(1) \# 1$ & 106.62(7) & $C(1)-C(5)-C(4)$ & 107.74(19) \\
\hline $\mathrm{C}(3) \# 1-\mathrm{U}(1)-\mathrm{C}(1) \# 1$ & $49.49(6)$ & $C(1)-C(5)-C(10)$ & $125.3(2)$ \\
\hline $\mathrm{C}(3)-\mathrm{U}(1)-\mathrm{C}(1) \# 1$ & $127.47(6)$ & $C(4)-C(5)-C(10)$ & $126.9(2)$ \\
\hline $\mathrm{C}(4) \# 1-\mathrm{U}(1)-\mathrm{C}(1) \# 1$ & $49.40(7)$ & $\mathrm{C}(1)-\mathrm{C}(5)-\mathrm{U}(1)$ & $75.36(13)$ \\
\hline $\mathrm{C}(4)-\mathrm{U}(1)-\mathrm{C}(1) \# 1$ & $129.21(7)$ & $\mathrm{C}(4)-\mathrm{C}(5)-\mathrm{U}(1)$ & $74.70(13)$ \\
\hline $\mathrm{C}(2) \# 1-\mathrm{U}(1)-\mathrm{C}(1) \# 1$ & $30.20(6)$ & $\mathrm{C}(10)-\mathrm{C}(5)-\mathrm{U}(1)$ & $119.17(15)$ \\
\hline$C(2)-U(1)-C(1) \# 1$ & $146.45(6)$ & $\mathrm{N}(1)-\mathrm{C}(11)-\mathrm{C}(13)$ & $121.9(2)$ \\
\hline $\mathrm{C}(5)-\mathrm{U}(1)-\mathrm{C}(1) \# 1$ & $150.77(7)$ & $\mathrm{N}(1)-\mathrm{C}(11)-\mathrm{C}(12)$ & $122.2(2)$ \\
\hline $\mathrm{C}(5) \# 1-\mathrm{U}(1)-\mathrm{C}(1) \# 1$ & 29.91(7) & $\mathrm{C}(13)-\mathrm{C}(11)-\mathrm{C}(12)$ & $115.98(19)$ \\
\hline $\mathrm{C}(1)-\mathrm{U}(1)-\mathrm{C}(1) \# 1$ & $176.60(8)$ & $C(14)-C(13)-C(18)$ & $118.8(2)$ \\
\hline $\mathrm{C}(11)-\mathrm{N}(1)-\mathrm{U}(1)$ & $172.48(17)$ & $\mathrm{C}(14)-\mathrm{C}(13)-\mathrm{C}(11)$ & $119.0(2)$ \\
\hline$C(5)-C(1)-C(2)$ & $108.16(19)$ & $C(18)-C(13)-C(11)$ & $122.2(2)$ \\
\hline$C(5)-C(1)-C(6)$ & $125.31(19)$ & $\mathrm{C}(15)-\mathrm{C}(14)-\mathrm{C}(13)$ & $119.3(2)$ \\
\hline
\end{tabular}




\begin{tabular}{|ll|l|}
\hline$F(1)-C(15)-C(14)$ & $120.3(2)$ & \\
$F(1)-C(15)-C(16)$ & $118.0(2)$ & \\
$C(14)-C(15)-C(16)$ & $121.7(2)$ & \\
$F(2)-C(16)-C(17)$ & $121.1(2)$ & \\
$F(2)-C(16)-C(15)$ & $120.1(2)$ & \\
C(17)-C(16)-C(15) & $118.8(2)$ & \\
F(3)-C(17)-C(18) & $120.0(2)$ & \\
F(3)-C(17)-C(16) & $118.5(2)$ & \\
C(18)-C(17)-C(16) & $121.5(2)$ & \\
C(17)-C(18)-C(13) & \\
\hline
\end{tabular}


Figure S5: Thermal Ellipsoid Plot of $\left(\mathrm{C}_{5} \mathrm{Me}_{5}\right)_{2} \mathrm{U}\left[-\mathrm{N}=\mathrm{C}\left(\mathrm{CH}_{3}\right)\left(\mathrm{C}_{6} \mathrm{~F}_{5}\right)\right]_{2}(\mathbf{1 1})$ with Ellipsoids Projected at the $50 \%$ Probability Level.

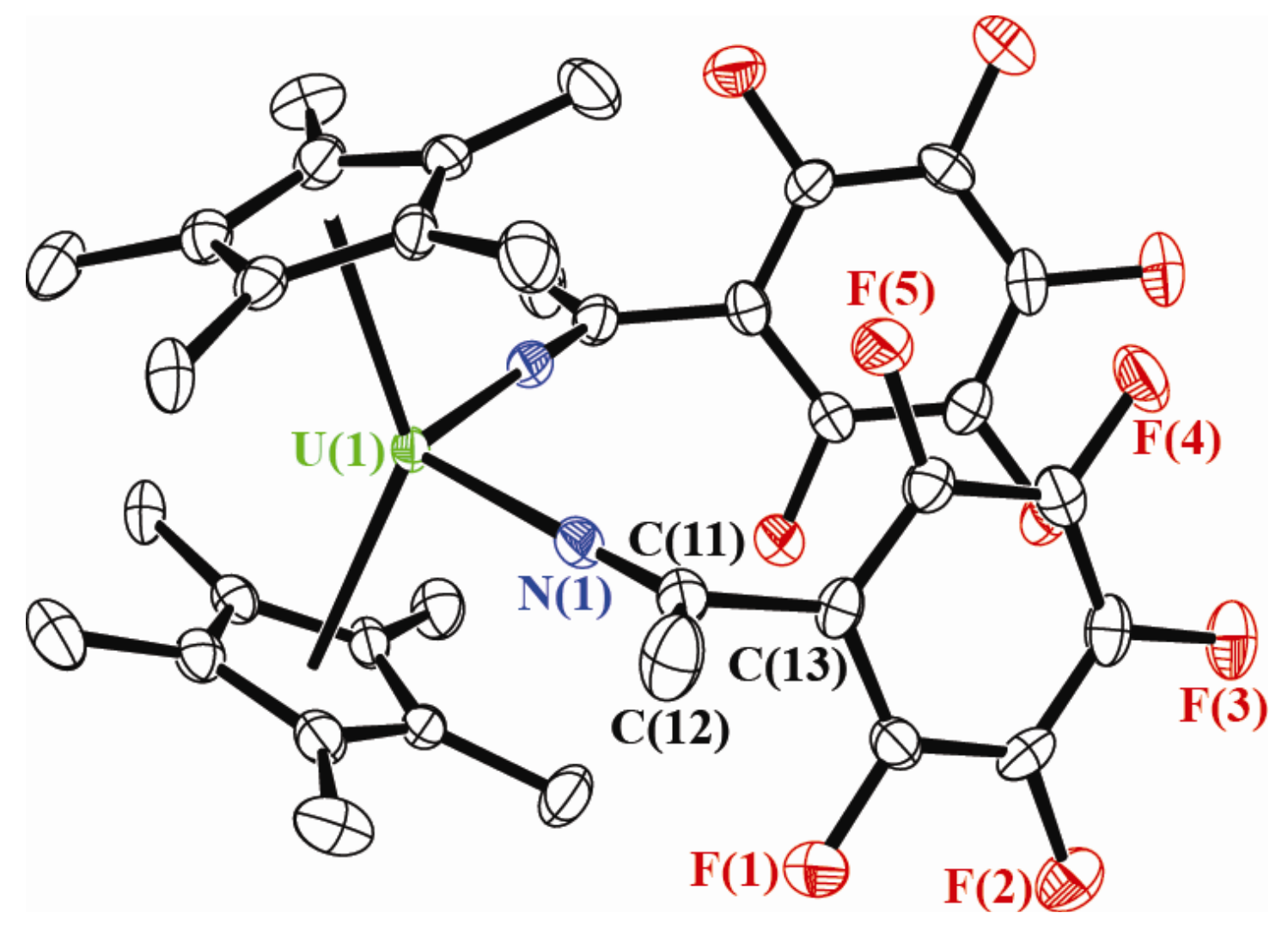


Table S13: Crystal Data and Structure Refinement for $\left(\mathrm{C}_{5} \mathrm{Me}_{5}\right)_{2} \mathrm{U}\left[-\mathrm{N}=\mathrm{C}\left(\mathrm{CH}_{3}\right)\left(\mathrm{C}_{6} \mathrm{~F}_{5}\right)\right]_{2}(\mathbf{1 1})$.

\begin{tabular}{|c|c|c|}
\hline Empirical formula & \multicolumn{2}{|l|}{$\mathrm{C}_{36} \mathrm{H}_{36} \mathrm{~F}_{10} \mathrm{~N}_{2} \mathrm{U}$} \\
\hline Formula weight & \multicolumn{2}{|l|}{924.70} \\
\hline Temperature & \multicolumn{2}{|l|}{$141(2) \mathrm{K}$} \\
\hline Wavelength & \multicolumn{2}{|l|}{$0.71073 \AA$} \\
\hline Crystal system & \multicolumn{2}{|l|}{ orthorhombic } \\
\hline Space group & \multicolumn{2}{|l|}{$\mathrm{P} \operatorname{ccn}$} \\
\hline \multirow[t]{3}{*}{ Unit cell dimensions } & $a=15.9821(4) \AA$ & $\alpha=90^{\circ}$ \\
\hline & $\mathrm{b}=16.3139(4) \AA$ & $\beta=90^{\circ}$ \\
\hline & $c=26.9285(6) \AA$ & $\gamma=90^{\circ}$ \\
\hline Volume & \multicolumn{2}{|l|}{$7021.1(3) \AA^{3}$} \\
\hline $\mathrm{Z}$ & \multicolumn{2}{|l|}{8} \\
\hline Density (calculated) & \multicolumn{2}{|l|}{$1.750 \mathrm{Mg} / \mathrm{m}^{3}$} \\
\hline Absorption coefficient & \multicolumn{2}{|l|}{$4.705 \mathrm{~mm}^{-1}$} \\
\hline $\mathrm{F}(000)$ & \multicolumn{2}{|l|}{3584} \\
\hline Crystal size & \multicolumn{2}{|c|}{$0.18 \times 0.12 \times 0.04 \mathrm{~mm}^{3}$} \\
\hline$\Theta$ Range for data collection & \multicolumn{2}{|l|}{1.78 to $25.31^{\circ}$} \\
\hline Index ranges & \multicolumn{2}{|c|}{$-19 \leq \mathrm{h} \leq 19,-19 \leq \mathrm{k} \leq 19,-22 \leq 1 \leq 32$} \\
\hline Reflections collected & \multicolumn{2}{|l|}{36815} \\
\hline Independent reflections & \multicolumn{2}{|c|}{$6408[\mathrm{R}(\mathrm{int})=0.0362]$} \\
\hline Completeness to $\Theta=25.31^{\circ}$ & \multicolumn{2}{|l|}{$99.9 \%$} \\
\hline Max. and min. transmission & \multicolumn{2}{|l|}{0.8341 and 0.4847} \\
\hline Refinement method & \multicolumn{2}{|c|}{ Full-matrix least-squares on $\mathrm{F}^{2}$} \\
\hline Data / restraints / parameters & \multicolumn{2}{|l|}{$6408 / 0 / 395$} \\
\hline Goodness-of-fit on $\mathrm{F}^{2}$ & \multicolumn{2}{|l|}{1.559} \\
\hline Final $R$ indices $[\mathrm{I}>2 \sigma(\mathrm{I})]$ & \multicolumn{2}{|c|}{$\mathrm{R} 1=0.0318, \mathrm{wR} 2=0.0931$} \\
\hline $\mathrm{R}$ indices (all data) & \multicolumn{2}{|c|}{$\mathrm{R} 1=0.0544, \mathrm{wR} 2=0.1002$} \\
\hline Largest diff. peak and hole & \multicolumn{2}{|c|}{1.387 and -1.367 e. $\AA^{-3}$} \\
\hline
\end{tabular}


Table S14: Bond Distances $[\AA]$ and Angles $\left[^{\circ}\right]$ for $\left(\mathrm{C}_{5} \mathrm{Me}_{5}\right)_{2} \mathrm{U}\left[-\mathrm{N}=\mathrm{C}\left(\mathrm{CH}_{3}\right)\left(\mathrm{C}_{6} \mathrm{~F}_{5}\right)\right]_{2}(\mathbf{1 1})$.

\begin{tabular}{|c|c|c|c|}
\hline $\mathrm{U}(1)-\mathrm{N}(1) \# 1$ & $2.204(5)$ & $\mathrm{N}(2)-\mathrm{C}(29)$ & $1.248(7)$ \\
\hline $\mathrm{U}(1)-\mathrm{N}(1)$ & $2.204(5)$ & $C(1)-C(2)$ & 1.4200 \\
\hline $\mathrm{U}(1)-\mathrm{C}(3)$ & $2.767(2)$ & $C(1)-C(5)$ & 1.4200 \\
\hline $\mathrm{U}(1)-\mathrm{C}(3) \# 1$ & $2.767(4)$ & $\mathrm{C}(1)-\mathrm{C}(6)$ & 1.5095 \\
\hline $\mathrm{U}(1)-\mathrm{C}(2)$ & $2.774(2)$ & $\mathrm{C}(2)-\mathrm{C}(3)$ & 1.4200 \\
\hline $\mathrm{U}(1)-\mathrm{C}(2) \# 1$ & $2.774(5)$ & $\mathrm{C}(2)-\mathrm{C}(7)$ & 1.5095 \\
\hline $\mathrm{U}(1)-\mathrm{C}(4) \# 1$ & $2.7893(19)$ & $C(3)-C(4)$ & 1.4200 \\
\hline $\mathrm{U}(1)-\mathrm{C}(4)$ & $2.789(2)$ & $\mathrm{C}(3)-\mathrm{C}(8)$ & 1.5095 \\
\hline $\mathrm{U}(1)-\mathrm{C}(1)$ & $2.800(2)$ & $C(4)-C(5)$ & 1.4200 \\
\hline $\mathrm{U}(1)-\mathrm{C}(1) \# 1$ & $2.800(2)$ & $\mathrm{C}(4)-\mathrm{C}(9)$ & 1.5095 \\
\hline $\mathrm{U}(1)-\mathrm{C}(5) \# 1$ & $2.810(2)$ & $C(5)-C(10)$ & 1.5095 \\
\hline $\mathrm{U}(1)-\mathrm{C}(5)$ & $2.810(2)$ & $\mathrm{C}(11)-\mathrm{C}(12)$ & $1.495(7)$ \\
\hline $\mathrm{U}(2)-\mathrm{N}(2) \# 2$ & $2.199(5)$ & $C(11)-C(13)$ & $1.535(8)$ \\
\hline $\mathrm{U}(2)-\mathrm{N}(2)$ & $2.199(5)$ & $\mathrm{C}(13)-\mathrm{C}(14)$ & $1.390(7)$ \\
\hline $\mathrm{U}(2)-\mathrm{C}(20) \# 2$ & $2.753(5)$ & $C(13)-C(18)$ & $1.388(7)$ \\
\hline $\mathrm{U}(2)-\mathrm{C}(20)$ & $2.753(2)$ & $C(14)-C(15)$ & $1.366(8)$ \\
\hline $\mathrm{U}(2)-\mathrm{C}(19) \# 2$ & $2.754(2)$ & $C(15)-C(16)$ & $1.387(8)$ \\
\hline $\mathrm{U}(2)-\mathrm{C}(19)$ & $2.754(2)$ & $C(16)-C(17)$ & $1.382(8)$ \\
\hline $\mathrm{U}(2)-\mathrm{C}(21) \# 2$ & $2.784(4)$ & $\mathrm{C}(17)-\mathrm{C}(18)$ & $1.363(7)$ \\
\hline $\mathrm{U}(2)-\mathrm{C}(21)$ & $2.784(2)$ & $C(19)-C(20)$ & 1.4200 \\
\hline $\mathrm{U}(2)-\mathrm{C}(23)$ & $2.784(2)$ & $C(19)-C(23)$ & 1.4200 \\
\hline $\mathrm{U}(2)-\mathrm{C}(23) \# 2$ & $2.784(3)$ & $C(19)-C(24)$ & 1.5095 \\
\hline $\mathrm{U}(2)-\mathrm{C}(22) \# 2$ & $2.803(2)$ & $C(20)-C(21)$ & 1.4200 \\
\hline $\mathrm{U}(2)-\mathrm{C}(22)$ & $2.803(2)$ & $C(20)-C(25)$ & 1.5095 \\
\hline $\mathrm{F}(1)-\mathrm{C}(14)$ & $1.350(6)$ & $C(21)-C(22)$ & 1.4200 \\
\hline $\mathrm{F}(2)-\mathrm{C}(15)$ & $1.345(6)$ & $\mathrm{C}(21)-\mathrm{C}(26)$ & 1.5095 \\
\hline $\mathrm{F}(3)-\mathrm{C}(16)$ & $1.343(6)$ & $\mathrm{C}(22)-\mathrm{C}(23)$ & 1.4200 \\
\hline $\mathrm{F}(4)-\mathrm{C}(17)$ & $1.343(6)$ & $\mathrm{C}(22)-\mathrm{C}(27)$ & 1.5095 \\
\hline $\mathrm{F}(5)-\mathrm{C}(18)$ & $1.351(6)$ & $\mathrm{C}(23)-\mathrm{C}(28)$ & 1.5095 \\
\hline $\mathrm{F}(6)-\mathrm{C}(32)$ & $1.355(6)$ & $\mathrm{C}(29)-\mathrm{C}(30)$ & $1.511(7)$ \\
\hline $\mathrm{F}(7)-\mathrm{C}(33)$ & $1.349(6)$ & $\mathrm{C}(29)-\mathrm{C}(31)$ & $1.531(8)$ \\
\hline $\mathrm{F}(8)-\mathrm{C}(34)$ & $1.339(6)$ & $\mathrm{C}(31)-\mathrm{C}(36)$ & $1.380(7)$ \\
\hline $\mathrm{F}(9)-\mathrm{C}(35)$ & $1.353(6)$ & $\mathrm{C}(31)-\mathrm{C}(32)$ & 1.391(7) \\
\hline $\mathrm{F}(10)-\mathrm{C}(36)$ & $1.344(6)$ & $\mathrm{C}(32)-\mathrm{C}(33)$ & $1.376(8)$ \\
\hline
\end{tabular}




\begin{tabular}{|c|c|c|c|}
\hline $\mathrm{N}(1)-\mathrm{C}(11)$ & $1.243(7)$ & $\mathrm{C}(33)-\mathrm{C}(34)$ & $1.377(8)$ \\
\hline$C(34)-C(35)$ & $1.389(9)$ & $\mathrm{C}(2)-\mathrm{U}(1)-\mathrm{C}(1)$ & 29.5 \\
\hline \multirow[t]{2}{*}{$C(35)-C(36)$} & $1.351(8)$ & $\mathrm{C}(2) \# 1-\mathrm{U}(1)-\mathrm{C}(1)$ & $116.84(10)$ \\
\hline & & $\mathrm{C}(4) \# 1-\mathrm{U}(1)-\mathrm{C}(1)$ & $128.41(13)$ \\
\hline $\mathrm{N}(1) \# 1-\mathrm{U}(1)-\mathrm{N}(1)$ & $104.8(2)$ & $\mathrm{C}(4)-\mathrm{U}(1)-\mathrm{C}(1)$ & 48.5 \\
\hline $\mathrm{N}(1) \# 1-\mathrm{U}(1)-\mathrm{C}(3)$ & $94.65(11)$ & $\mathrm{N}(1) \# 1-\mathrm{U}(1)-\mathrm{C}(1) \# 1$ & $79.34(11)$ \\
\hline $\mathrm{N}(1)-\mathrm{U}(1)-\mathrm{C}(3)$ & $125.98(11)$ & $\mathrm{N}(1)-\mathrm{U}(1)-\mathrm{C}(1) \# 1$ & $123.62(11)$ \\
\hline $\mathrm{N}(1) \# 1-\mathrm{U}(1)-\mathrm{C}(3) \# 1$ & $125.98(14)$ & $C(3)-U(1)-C(1) \# 1$ & $109.17(6)$ \\
\hline $\mathrm{N}(1)-\mathrm{U}(1)-\mathrm{C}(3) \# 1$ & $94.65(15)$ & $\mathrm{C}(3) \# 1-\mathrm{U}(1)-\mathrm{C}(1) \# 1$ & $48.75(11)$ \\
\hline $\mathrm{C}(3)-\mathrm{U}(1)-\mathrm{C}(3) \# 1$ & $113.56(11)$ & $\mathrm{C}(2)-\mathrm{U}(1)-\mathrm{C}(1) \# 1$ & $116.84(8)$ \\
\hline $\mathrm{N}(1) \# 1-\mathrm{U}(1)-\mathrm{C}(2)$ & $123.71(11)$ & $\mathrm{C}(2) \# 1-\mathrm{U}(1)-\mathrm{C}(1) \# 1$ & $29.52(8)$ \\
\hline $\mathrm{N}(1)-\mathrm{U}(1)-\mathrm{C}(2)$ & $106.91(12)$ & $\mathrm{C}(4) \# 1-\mathrm{U}(1)-\mathrm{C}(1) \# 1$ & $48.54(13)$ \\
\hline $\mathrm{C}(3)-\mathrm{U}(1)-\mathrm{C}(2)$ & 29.7 & $\mathrm{C}(4)-\mathrm{U}(1)-\mathrm{C}(1) \# 1$ & 128.4 \\
\hline $\mathrm{C}(3) \# 1-\mathrm{U}(1)-\mathrm{C}(2)$ & $96.27(10)$ & $\mathrm{C}(1)-\mathrm{U}(1)-\mathrm{C}(1) \# 1$ & $144.83(9)$ \\
\hline $\mathrm{N}(1) \# 1-\mathrm{U}(1)-\mathrm{C}(2) \# 1$ & $106.91(15)$ & $\mathrm{N}(1) \# 1-\mathrm{U}(1)-\mathrm{C}(5) \# 1$ & $79.38(16)$ \\
\hline $\mathrm{N}(1)-\mathrm{U}(1)-\mathrm{C}(2) \# 1$ & $123.71(14)$ & $\mathrm{N}(1)-\mathrm{U}(1)-\mathrm{C}(5) \# 1$ & $94.94(16)$ \\
\hline $\mathrm{C}(3)-\mathrm{U}(1)-\mathrm{C}(2) \# 1$ & $96.27(10)$ & $\mathrm{C}(3)-\mathrm{U}(1)-\mathrm{C}(5) \# 1$ & $138.48(13)$ \\
\hline $\mathrm{C}(3) \# 1-\mathrm{U}(1)-\mathrm{C}(2) \# 1$ & 29.7 & $\mathrm{C}(3) \# 1-\mathrm{U}(1)-\mathrm{C}(5) \# 1$ & $48.66(6)$ \\
\hline $\mathrm{C}(2)-\mathrm{U}(1)-\mathrm{C}(2) \# 1$ & $92.25(9)$ & $\mathrm{C}(2)-\mathrm{U}(1)-\mathrm{C}(5) \# 1$ & $140.62(13)$ \\
\hline $\mathrm{N}(1) \# 1-\mathrm{U}(1)-\mathrm{C}(4) \# 1$ & $106.81(16)$ & $\mathrm{C}(2) \# 1-\mathrm{U}(1)-\mathrm{C}(5) \# 1$ & $48.60(7)$ \\
\hline $\mathrm{N}(1)-\mathrm{U}(1)-\mathrm{C}(4) \# 1$ & $78.05(16)$ & $\mathrm{C}(4) \# 1-\mathrm{U}(1)-\mathrm{C}(5) \# 1$ & 29.4 \\
\hline $\mathrm{C}(3)-\mathrm{U}(1)-\mathrm{C}(4) \# 1$ & $142.71(14)$ & $\mathrm{C}(4)-\mathrm{U}(1)-\mathrm{C}(5) \# 1$ & $151.91(13)$ \\
\hline $\mathrm{C}(3) \# 1-\mathrm{U}(1)-\mathrm{C}(4) \# 1$ & 29.6 & $\mathrm{C}(1)-\mathrm{U}(1)-\mathrm{C}(5) \# 1$ & $157.00(13)$ \\
\hline $\mathrm{C}(2)-\mathrm{U}(1)-\mathrm{C}(4) \# 1$ & $124.43(13)$ & $\mathrm{C}(1) \# 1-\mathrm{U}(1)-\mathrm{C}(5) \# 1$ & $29.33(13)$ \\
\hline $\mathrm{C}(2) \# 1-\mathrm{U}(1)-\mathrm{C}(4) \# 1$ & $48.79(8)$ & $\mathrm{N}(1) \# 1-\mathrm{U}(1)-\mathrm{C}(5)$ & $94.94(11)$ \\
\hline $\mathrm{N}(1) \# 1-\mathrm{U}(1)-\mathrm{C}(4)$ & $78.05(11)$ & $\mathrm{N}(1)-\mathrm{U}(1)-\mathrm{C}(5)$ & $79.38(11)$ \\
\hline $\mathrm{N}(1)-\mathrm{U}(1)-\mathrm{C}(4)$ & $106.81(11)$ & $\mathrm{C}(3)-\mathrm{U}(1)-\mathrm{C}(5)$ & 48.7 \\
\hline $\mathrm{C}(3)-\mathrm{U}(1)-\mathrm{C}(4)$ & 29.6 & $\mathrm{C}(3) \# 1-\mathrm{U}(1)-\mathrm{C}(5)$ & $138.48(11)$ \\
\hline $\mathrm{C}(3) \# 1-\mathrm{U}(1)-\mathrm{C}(4)$ & $142.71(10)$ & $\mathrm{C}(2)-\mathrm{U}(1)-\mathrm{C}(5)$ & 48.6 \\
\hline $\mathrm{C}(2)-\mathrm{U}(1)-\mathrm{C}(4)$ & 48.8 & $\mathrm{C}(2) \# 1-\mathrm{U}(1)-\mathrm{C}(5)$ & $140.62(8)$ \\
\hline $\mathrm{C}(2) \# 1-\mathrm{U}(1)-\mathrm{C}(4)$ & $124.43(10)$ & $\mathrm{C}(4) \# 1-\mathrm{U}(1)-\mathrm{C}(5)$ & $151.91(12)$ \\
\hline C(4)\#1-U(1)-C(4) & $172.27(13)$ & $\mathrm{C}(4)-\mathrm{U}(1)-\mathrm{C}(5)$ & 29.4 \\
\hline $\mathrm{N}(1) \# 1-\mathrm{U}(1)-\mathrm{C}(1)$ & $123.62(11)$ & $\mathrm{C}(1)-\mathrm{U}(1)-\mathrm{C}(5)$ & 29.3 \\
\hline $\mathrm{N}(1)-\mathrm{U}(1)-\mathrm{C}(1)$ & $79.34(11)$ & $\mathrm{C}(1) \# 1-\mathrm{U}(1)-\mathrm{C}(5)$ & 157.0 \\
\hline $\mathrm{C}(3)-\mathrm{U}(1)-\mathrm{C}(1)$ & 48.7 & $\mathrm{C}(5) \# 1-\mathrm{U}(1)-\mathrm{C}(5)$ & $170.77(13)$ \\
\hline
\end{tabular}




\begin{tabular}{|c|c|c|c|}
\hline $\mathrm{C}(3) \# 1-\mathrm{U}(1)-\mathrm{C}(1)$ & $109.17(12)$ & $\mathrm{N}(2) \# 2-\mathrm{U}(2)-\mathrm{N}(2)$ & $106.1(2)$ \\
\hline $\mathrm{N}(2) \# 2-\mathrm{U}(2)-\mathrm{C}(20) \# 2$ & $123.72(15)$ & & \\
\hline $\mathrm{N}(2)-\mathrm{U}(2)-\mathrm{C}(20) \# 2$ & 106.34(16) & $\mathrm{N}(2) \# 2-\mathrm{U}(2)-\mathrm{C}(23) \# 2$ & $94.54(17)$ \\
\hline $\mathrm{N}(2) \# 2-\mathrm{U}(2)-\mathrm{C}(20)$ & $106.34(12)$ & $\mathrm{N}(2)-\mathrm{U}(2)-\mathrm{C}(23) \# 2$ & $79.66(17)$ \\
\hline $\mathrm{N}(2)-\mathrm{U}(2)-\mathrm{C}(20)$ & $123.72(12)$ & $\mathrm{C}(20) \# 2-\mathrm{U}(2)-\mathrm{C}(23) \# 2$ & $49.02(8)$ \\
\hline $\mathrm{C}(20) \# 2-\mathrm{U}(2)-\mathrm{C}(20)$ & $91.85(10)$ & $\mathrm{C}(20)-\mathrm{U}(2)-\mathrm{C}(23) \# 2$ & $140.51(14)$ \\
\hline N(2)\#2-U(2)-C(19)\#2 & $123.66(12)$ & $\mathrm{C}(19) \# 2-\mathrm{U}(2)-\mathrm{C}(23) \# 2$ & 29.71(14) \\
\hline $\mathrm{N}(2)-\mathrm{U}(2)-\mathrm{C}(19) \# 2$ & $78.63(12)$ & $\mathrm{C}(19)-\mathrm{U}(2)-\mathrm{C}(23) \# 2$ & $156.65(15)$ \\
\hline $\mathrm{C}(20) \# 2-\mathrm{U}(2)-\mathrm{C}(19) \# 2$ & $29.89(9)$ & $\mathrm{C}(21) \# 2-\mathrm{U}(2)-\mathrm{C}(23) \# 2$ & $48.74(7)$ \\
\hline $\mathrm{C}(20)-\mathrm{U}(2)-\mathrm{C}(19) \# 2$ & $116.99(11)$ & $\mathrm{C}(21)-\mathrm{U}(2)-\mathrm{C}(23) \# 2$ & $138.76(14)$ \\
\hline N(2)\#2-U(2)-C(19) & $78.63(12)$ & $\mathrm{C}(23)-\mathrm{U}(2)-\mathrm{C}(23) \# 2$ & $170.43(14)$ \\
\hline $\mathrm{N}(2)-\mathrm{U}(2)-\mathrm{C}(19)$ & $123.66(12)$ & $\mathrm{N}(2) \# 2-\mathrm{U}(2)-\mathrm{C}(22) \# 2$ & $77.97(17)$ \\
\hline $\mathrm{C}(20) \# 2-\mathrm{U}(2)-\mathrm{C}(19)$ & $116.99(12)$ & $\mathrm{N}(2)-\mathrm{U}(2)-\mathrm{C}(22) \# 2$ & $107.53(17)$ \\
\hline $\mathrm{C}(20)-\mathrm{U}(2)-\mathrm{C}(19)$ & 29.9 & $C(20) \# 2-U(2)-C(22) \# 2$ & $48.84(9)$ \\
\hline C(19)\#2-U(2)-C(19) & $145.44(12)$ & $\mathrm{C}(20)-\mathrm{U}(2)-\mathrm{C}(22) \# 2$ & $123.42(14)$ \\
\hline $\mathrm{N}(2) \# 2-\mathrm{U}(2)-\mathrm{C}(21) \# 2$ & $94.62(16)$ & $\mathrm{C}(19) \# 2-\mathrm{U}(2)-\mathrm{C}(22) \# 2$ & $48.84(14)$ \\
\hline $\mathrm{N}(2)-\mathrm{U}(2)-\mathrm{C}(21) \# 2$ & $125.88(15)$ & $C(19)-U(2)-C(22) \# 2$ & $127.76(15)$ \\
\hline $\mathrm{C}(20) \# 2-\mathrm{U}(2)-\mathrm{C}(21) \# 2$ & 29.71(6) & $\mathrm{C}(21) \# 2-\mathrm{U}(2)-\mathrm{C}(22) \# 2$ & 29.4 \\
\hline $\mathrm{C}(20)-\mathrm{U}(2)-\mathrm{C}(21) \# 2$ & $95.58(11)$ & $\mathrm{C}(21)-\mathrm{U}(2)-\mathrm{C}(22) \# 2$ & $141.75(15)$ \\
\hline $\mathrm{C}(19) \# 2-\mathrm{U}(2)-\mathrm{C}(21) \# 2$ & $49.03(12)$ & $\mathrm{C}(23)-\mathrm{U}(2)-\mathrm{C}(22) \# 2$ & $152.09(13)$ \\
\hline $\mathrm{C}(19)-\mathrm{U}(2)-\mathrm{C}(21) \# 2$ & $109.05(13)$ & $C(23) \# 2-U(2)-C(22) \# 2$ & 29.4 \\
\hline $\mathrm{N}(2) \# 2-\mathrm{U}(2)-\mathrm{C}(21)$ & $125.88(11)$ & $\mathrm{N}(2) \# 2-\mathrm{U}(2)-\mathrm{C}(22)$ & $107.53(11)$ \\
\hline $\mathrm{N}(2)-\mathrm{U}(2)-\mathrm{C}(21)$ & $94.62(12)$ & $\mathrm{N}(2)-\mathrm{U}(2)-\mathrm{C}(22)$ & $77.97(11)$ \\
\hline $\mathrm{C}(20) \# 2-\mathrm{U}(2)-\mathrm{C}(21)$ & $95.58(11)$ & $\mathrm{C}(20) \# 2-\mathrm{U}(2)-\mathrm{C}(22)$ & $123.42(11)$ \\
\hline $\mathrm{C}(20)-\mathrm{U}(2)-\mathrm{C}(21)$ & 29.7 & $C(20)-U(2)-C(22)$ & 48.8 \\
\hline $\mathrm{C}(19) \# 2-\mathrm{U}(2)-\mathrm{C}(21)$ & $109.05(8)$ & $\mathrm{C}(19) \# 2-\mathrm{U}(2)-\mathrm{C}(22)$ & 127.8 \\
\hline $\mathrm{C}(19)-\mathrm{U}(2)-\mathrm{C}(21)$ & 49.0 & $C(19)-U(2)-C(22)$ & 48.8 \\
\hline $\mathrm{C}(21) \# 2-\mathrm{U}(2)-\mathrm{C}(21)$ & $112.66(12)$ & $\mathrm{C}(21) \# 2-\mathrm{U}(2)-\mathrm{C}(22)$ & $141.75(12)$ \\
\hline $\mathrm{N}(2) \# 2-\mathrm{U}(2)-\mathrm{C}(23)$ & $79.66(11)$ & $C(21)-U(2)-C(22)$ & 29.4 \\
\hline $\mathrm{N}(2)-\mathrm{U}(2)-\mathrm{C}(23)$ & $94.54(12)$ & $C(23)-U(2)-C(22)$ & 29.4 \\
\hline $\mathrm{C}(20) \# 2-\mathrm{U}(2)-\mathrm{C}(23)$ & $140.51(9)$ & $\mathrm{C}(23) \# 2-\mathrm{U}(2)-\mathrm{C}(22)$ & $152.09(14)$ \\
\hline $\mathrm{C}(20)-\mathrm{U}(2)-\mathrm{C}(23)$ & 49.0 & $\mathrm{C}(22) \# 2-\mathrm{U}(2)-\mathrm{C}(22)$ & $171.16(15)$ \\
\hline $\mathrm{C}(19) \# 2-\mathrm{U}(2)-\mathrm{C}(23)$ & $156.65(5)$ & $\mathrm{C}(11)-\mathrm{N}(1)-\mathrm{U}(1)$ & $178.4(4)$ \\
\hline $\mathrm{C}(19)-\mathrm{U}(2)-\mathrm{C}(23)$ & 29.7 & $\mathrm{C}(29)-\mathrm{N}(2)-\mathrm{U}(2)$ & $176.3(4)$ \\
\hline \multirow[t]{2}{*}{$\mathrm{C}(21) \# 2-\mathrm{U}(2)-\mathrm{C}(23)$} & $138.76(12)$ & $C(2)-C(1)-C(5)$ & 108.0 \\
\hline & & $\mathrm{C}(2)-\mathrm{C}(1)-\mathrm{C}(6)$ & 126.0 \\
\hline
\end{tabular}




\begin{tabular}{|c|c|c|c|}
\hline $\mathrm{C}(21)-\mathrm{U}(2)-\mathrm{C}(23)$ & 48.7 & $C(5)-C(1)-C(6)$ & 126.0 \\
\hline$C(2)-C(1)-U(1)$ & $74.21(7)$ & & \\
\hline $\mathrm{C}(5)-\mathrm{C}(1)-\mathrm{U}(1)$ & $75.71(7)$ & $C(15)-C(14)-C(13)$ & $122.9(5)$ \\
\hline$C(6)-C(1)-U(1)$ & $116.19(6)$ & $\mathrm{F}(2)-\mathrm{C}(15)-\mathrm{C}(14)$ & $121.1(5)$ \\
\hline$C(1)-C(2)-C(3)$ & 108.0 & $\mathrm{~F}(2)-\mathrm{C}(15)-\mathrm{C}(16)$ & $119.5(5)$ \\
\hline$C(1)-C(2)-C(7)$ & 126.0 & $C(14)-C(15)-C(16)$ & $119.3(5)$ \\
\hline$C(3)-C(2)-C(7)$ & 126.0 & $F(3)-C(16)-C(17)$ & $120.2(5)$ \\
\hline$C(1)-C(2)-U(1)$ & $76.27(7)$ & $F(3)-C(16)-C(15)$ & $120.4(5)$ \\
\hline $\mathrm{C}(3)-\mathrm{C}(2)-\mathrm{U}(1)$ & $74.89(7)$ & $C(17)-C(16)-C(15)$ & $119.4(5)$ \\
\hline$C(7)-C(2)-U(1)$ & $115.06(7)$ & $F(4)-C(17)-C(18)$ & $121.5(5)$ \\
\hline$C(4)-C(3)-C(2)$ & 108.0 & $F(4)-C(17)-C(16)$ & $118.9(5)$ \\
\hline $\mathrm{C}(4)-\mathrm{C}(3)-\mathrm{C}(8)$ & 126.0 & $C(18)-C(17)-C(16)$ & $119.6(5)$ \\
\hline $\mathrm{C}(2)-\mathrm{C}(3)-\mathrm{C}(8)$ & 126.0 & $\mathrm{~F}(5)-\mathrm{C}(18)-\mathrm{C}(17)$ & $117.7(5)$ \\
\hline $\mathrm{C}(4)-\mathrm{C}(3)-\mathrm{U}(1)$ & $76.07(7)$ & $F(5)-C(18)-C(13)$ & $119.4(5)$ \\
\hline$C(2)-C(3)-U(1)$ & $75.41(7)$ & $\mathrm{C}(17)-\mathrm{C}(18)-\mathrm{C}(13)$ & $122.9(5)$ \\
\hline $\mathrm{C}(8)-\mathrm{C}(3)-\mathrm{U}(1)$ & $114.78(7)$ & $C(20)-C(19)-C(23)$ & 108.0 \\
\hline$C(3)-C(4)-C(5)$ & 108.0 & $\mathrm{C}(20)-\mathrm{C}(19)-\mathrm{C}(24)$ & 126.0 \\
\hline $\mathrm{C}(3)-\mathrm{C}(4)-\mathrm{C}(9)$ & 126.0 & $C(23)-C(19)-C(24)$ & 126.0 \\
\hline$C(5)-C(4)-C(9)$ & 126.0 & $\mathrm{C}(20)-\mathrm{C}(19)-\mathrm{U}(2)$ & $75.04(8)$ \\
\hline $\mathrm{C}(3)-\mathrm{C}(4)-\mathrm{U}(1)$ & $74.32(7)$ & $\mathrm{C}(23)-\mathrm{C}(19)-\mathrm{U}(2)$ & $76.35(7)$ \\
\hline $\mathrm{C}(5)-\mathrm{C}(4)-\mathrm{U}(1)$ & $76.10(7)$ & $\mathrm{C}(24)-\mathrm{C}(19)-\mathrm{U}(2)$ & $114.86(7)$ \\
\hline $\mathrm{C}(9)-\mathrm{C}(4)-\mathrm{U}(1)$ & $115.73(7)$ & $C(19)-C(20)-C(21)$ & 108.0 \\
\hline$C(4)-C(5)-C(1)$ & 108.0 & $C(19)-C(20)-C(25)$ & 126.0 \\
\hline$C(4)-C(5)-C(10)$ & 126.0 & $\mathrm{C}(21)-\mathrm{C}(20)-\mathrm{C}(25)$ & 126.0 \\
\hline$C(1)-C(5)-C(10)$ & 126.0 & $\mathrm{C}(19)-\mathrm{C}(20)-\mathrm{U}(2)$ & $75.07(8)$ \\
\hline $\mathrm{C}(4)-\mathrm{C}(5)-\mathrm{U}(1)$ & $74.52(7)$ & $\mathrm{C}(21)-\mathrm{C}(20)-\mathrm{U}(2)$ & $76.33(7)$ \\
\hline $\mathrm{C}(1)-\mathrm{C}(5)-\mathrm{U}(1)$ & $74.96(7)$ & $\mathrm{C}(25)-\mathrm{C}(20)-\mathrm{U}(2)$ & $114.84(7)$ \\
\hline $\mathrm{C}(10)-\mathrm{C}(5)-\mathrm{U}(1)$ & $116.60(6)$ & $\mathrm{C}(20)-\mathrm{C}(21)-\mathrm{C}(22)$ & 108.0 \\
\hline $\mathrm{N}(1)-\mathrm{C}(11)-\mathrm{C}(12)$ & $126.3(5)$ & $\mathrm{C}(20)-\mathrm{C}(21)-\mathrm{C}(26)$ & 126.0 \\
\hline $\mathrm{N}(1)-\mathrm{C}(11)-\mathrm{C}(13)$ & $119.7(5)$ & $\mathrm{C}(22)-\mathrm{C}(21)-\mathrm{C}(26)$ & 126.0 \\
\hline$C(12)-C(11)-C(13)$ & $113.9(5)$ & $\mathrm{C}(20)-\mathrm{C}(21)-\mathrm{U}(2)$ & $73.95(7)$ \\
\hline$C(14)-C(13)-C(18)$ & $115.8(5)$ & $\mathrm{C}(22)-\mathrm{C}(21)-\mathrm{U}(2)$ & $76.02(8)$ \\
\hline$C(14)-C(13)-C(11)$ & $121.1(5)$ & $\mathrm{C}(26)-\mathrm{C}(21)-\mathrm{U}(2)$ & $116.14(7)$ \\
\hline$C(18)-C(13)-C(11)$ & $123.1(5)$ & $\mathrm{C}(23)-\mathrm{C}(22)-\mathrm{C}(21)$ & 108.0 \\
\hline \multirow[t]{2}{*}{$F(1)-C(14)-C(15)$} & $117.7(5)$ & $C(23)-C(22)-C(27)$ & 126.0 \\
\hline & & $\mathrm{C}(21)-\mathrm{C}(22)-\mathrm{C}(27)$ & 126.0 \\
\hline
\end{tabular}




\begin{tabular}{|c|c|c|c|}
\hline $\mathrm{F}(1)-\mathrm{C}(14)-\mathrm{C}(13)$ & $119.4(5)$ & $\mathrm{C}(23)-\mathrm{C}(22)-\mathrm{U}(2)$ & $74.56(7)$ \\
\hline $\mathrm{C}(21)-\mathrm{C}(22)-\mathrm{U}(2)$ & $74.53(8)$ & & \\
\hline $\mathrm{C}(27)-\mathrm{C}(22)-\mathrm{U}(2)$ & $116.96(7)$ & & \\
\hline$C(22)-C(23)-C(19)$ & 108.0 & & \\
\hline $\mathrm{C}(22)-\mathrm{C}(23)-\mathrm{C}(28)$ & 126.0 & & \\
\hline$C(19)-C(23)-C(28)$ & 126.0 & & \\
\hline $\mathrm{C}(22)-\mathrm{C}(23)-\mathrm{U}(2)$ & $76.00(7)$ & & \\
\hline $\mathrm{C}(19)-\mathrm{C}(23)-\mathrm{U}(2)$ & $73.95(8)$ & & \\
\hline $\mathrm{C}(28)-\mathrm{C}(23)-\mathrm{U}(2)$ & $116.17(7)$ & & \\
\hline $\mathrm{N}(2)-\mathrm{C}(29)-\mathrm{C}(30)$ & $125.1(5)$ & & \\
\hline $\mathrm{N}(2)-\mathrm{C}(29)-\mathrm{C}(31)$ & $119.8(5)$ & & \\
\hline$C(30)-C(29)-C(31)$ & $115.1(5)$ & & \\
\hline $\mathrm{C}(36)-\mathrm{C}(31)-\mathrm{C}(32)$ & $116.1(5)$ & & \\
\hline$C(36)-C(31)-C(29)$ & $122.0(5)$ & & \\
\hline $\mathrm{C}(32)-\mathrm{C}(31)-\mathrm{C}(29)$ & $121.8(5)$ & & \\
\hline $\mathrm{F}(6)-\mathrm{C}(32)-\mathrm{C}(33)$ & $117.5(5)$ & & \\
\hline $\mathrm{F}(6)-\mathrm{C}(32)-\mathrm{C}(31)$ & $120.0(5)$ & & \\
\hline $\mathrm{C}(33)-\mathrm{C}(32)-\mathrm{C}(31)$ & $122.4(5)$ & & \\
\hline $\mathrm{F}(7)-\mathrm{C}(33)-\mathrm{C}(34)$ & $119.3(5)$ & & \\
\hline $\mathrm{F}(7)-\mathrm{C}(33)-\mathrm{C}(32)$ & $121.1(5)$ & & \\
\hline $\mathrm{C}(34)-\mathrm{C}(33)-\mathrm{C}(32)$ & $119.6(5)$ & & \\
\hline $\mathrm{F}(8)-\mathrm{C}(34)-\mathrm{C}(33)$ & $120.9(6)$ & & \\
\hline $\mathrm{F}(8)-\mathrm{C}(34)-\mathrm{C}(35)$ & $120.5(6)$ & & \\
\hline $\mathrm{C}(33)-\mathrm{C}(34)-\mathrm{C}(35)$ & $118.6(5)$ & & \\
\hline $\mathrm{F}(9)-\mathrm{C}(35)-\mathrm{C}(36)$ & $121.0(6)$ & & \\
\hline $\mathrm{F}(9)-\mathrm{C}(35)-\mathrm{C}(34)$ & $118.3(5)$ & & \\
\hline $\mathrm{C}(36)-\mathrm{C}(35)-\mathrm{C}(34)$ & $120.7(6)$ & & \\
\hline $\mathrm{F}(10)-\mathrm{C}(36)-\mathrm{C}(35)$ & $118.4(5)$ & & \\
\hline $\mathrm{F}(10)-\mathrm{C}(36)-\mathrm{C}(31)$ & $119.1(5)$ & & \\
\hline $\mathrm{C}(35)-\mathrm{C}(36)-\mathrm{C}(31)$ & $122.6(5)$ & & \\
\hline
\end{tabular}

